FAKUltät FÜR Physik UND Astronomie RUPREChT-KaRls-Universität HeIDELBERG

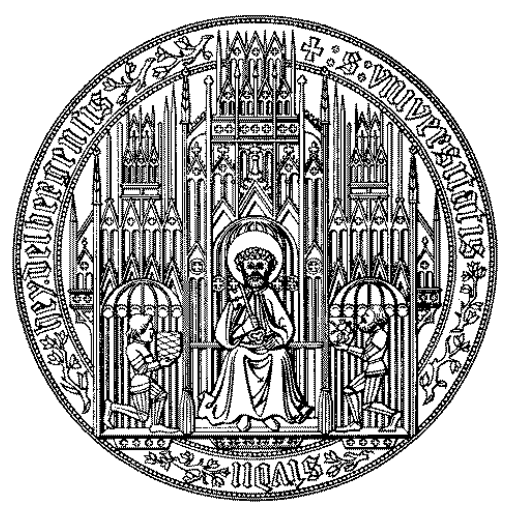

Diplomarbeit im Studiengang Physik

vorgelegt von

\title{
Guido Dirkes
}

aus Riesenbeck

Oktober 1999 



\title{
Messung der elektromagnetischen
}

\author{
Formfaktoren von Pionen \\ im SELEX Experiment
}

\author{
Die Diplomarbeit wurde von \\ Guido Dirkes \\ ausgeführt am \\ MAX-PlanCK-INSTITUT FÜR KERNPHYSIK \\ Heidelberg \\ unter der Betreuung von \\ Herrn Prof. Dr. Bogdan Povh.
}




\title{
Zusammenfassung
}

Die vorliegende Arbeit beschreibt die Analyse der Pion-Elektron Streudaten aus dem Fix-Target-Experiment SELEX am Fermilab mit Hinblick auf den elektromagnetischen Ladungsradius der Pionen. In der im September 1997 beendeten Strahlzeit wurden bei einer Triggerrate von 150 Ereignissen pro Sekunde 215 Millionen Kandidaten für elastische Streuereignisse aufgezeichnet. In mehreren Stufen der Datenreduktion wurden ca. 24000 Pion-Elektron-Streuereignisse extrahiert. Monte Carlo Simulationen erlaubten eine genaue Analyse der Akzeptanzfunktion, welcher aufgrund der besonderen Triggerkonfiguration eine besondere Bedeutung zukommt. Es wurden systematische Abhängigkeiten studiert und der Ladungsradius der Pionen aus dem gemessenen Formfaktor bestimmt.

\begin{abstract}
This work describes the analysis of pion-electron scattering data of the fixtrage-experiment SELEX at Fermilab with special respects to the pion charge radius. At an average rate of $150 \mathrm{~Hz}, 215$ million candidates for pion-electron elastic scattering were recorded during the fix target run until September 1997. A sample of 24000 pion-electron scattering events was extracted in the course of serveral data strips. Monte Carlo studies gave detailed knowledge of acceptance, which is important due to special trigger conditions. Systematics were studied and the pion mean square charge radius was determined.
\end{abstract}




\section{Inhalt}

1 Einleitung 1

2 Elastische Pion-Elektron-Streuung 3

2.1 Elastische Streuung . . . . . . . . . . . . . . . . 3

2.2 Formfaktoren und Ladungsradius . . . . . . . . . . . . . . . . . 5

2.3 Strahlungskorrekturen . . . . . . . . . . . . . . . 9

3 Das SELEX Experiment $\quad 14$

3.1 Allgemeine Ziele des SELEX Experiments . . . . . . . . . . . . . . . 14

3.1.1 Eigenschaften von charm-Baryonen . . . . . . . . . . . 14

3.1.2 Physikalische Schwerpunkte bei SELEX . . . . . . . . . . . 15

3.2 Das Spektrometer . . . . . . . . . . . . . . . 17

3.2.1 Das Strahlspektrometer und der Hyperonen-Strahl . . . . . 17

3.2.2 Die Targets und das Vertex-Spektrometer . . . . . . . . . 20

3.2.3 Das M1-Spektrometer . . . . . . . . . . . . 21

3.2.4 Das M2-Spektrometer . . . . . . . . . . . . . . 23

3.2.5 Das M3-Spektrometer . . . . . . . . . . . . 24

3.3 Teilchenidentifikation . . . . . . . . . . . . . . 24

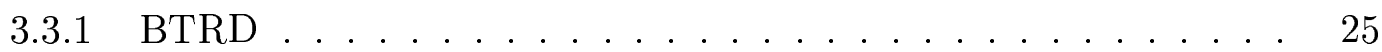

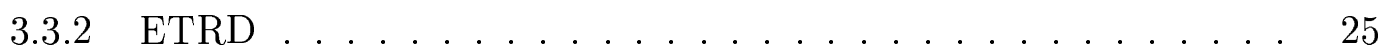

$3.3 .3 \mathrm{RICH} \ldots \ldots \ldots \ldots \ldots \ldots \ldots 26 \ldots \ldots \ldots \ldots$

3.3 .4 Die Kalorimeter . . . . . . . . . . . . . . . 27

3.4 Der Trigger . . . . . . . . . . . . . . . . . 28

3.4.1 Der Wechselwirkungszähler . . . . . . . . . . . . . 28

3.4 .2 Die Hodoskope . . . . . . . . . . . . . . . . . . . . . . . 29

3.4.3 Die Triggerlogik . . . . . . . . . . . . . . 30

4 Hodoskop- und Triggereffizienz $\quad 32$

4.1 Die Hodoskopanalyse . . . . . . . . . . . . . . . . . . . 33

4.1.1 Position der Hodoskopelemente . . . . . . . . . . . . . 33

4.1.2 Effizienz der Hodoskopelemente . . . . . . . . . . . . . . . 35

4.2 Die Triggereffizienz und Akzeptanzstudien . . . . . . . . . . . . . 36

4.2.1 GEANT Simulation . . . . . . . . . . . . . . . 37

4.2.2 Der $\mathrm{Q}^{2}$-abhängige Anteil der Triggereffizienz . . . . . . . . . 38

4.2 .3 Akzeptanzstudien . . . . . . . . . . . . . . . 40

5 Datenanalyse $\quad \mathbf{4 4}$

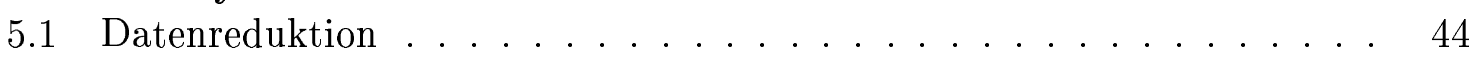

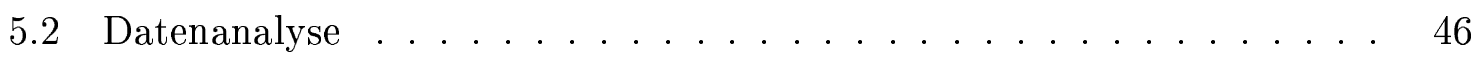

5.2 .1 Teilchenidentifikation . . . . . . . . . . . 47

5.2 .2 Vertex ......................... 48

5.2.3 Der Constrained Non-Linear Least Square Fit . . . . . . . . . . 49

5.2 .4 Selektion elastischer Streuereignisse . . . . . . . . . . . . . 50

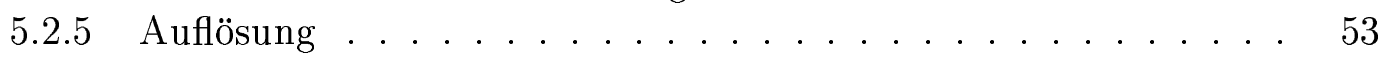

5.3 Der Radiusfit . . . . . . . . . . . . . . . . 54 
5.4 Systematische Studien . . . . . . . . . . . . . . 56

5.4.1 Abhängigkeiten von den Schnitten . . . . . . . . . . . 56

5.4 .2 Abhängigkeiten von den Fitgrenzen . . . . . . . . . . . . 56

5.4 .3 Statistischer und systematischer Fehler . . . . . . . . . 57

6 Ergebnis und Diskussion $\quad 60$

6.1 Diskussion der Analyse . . . . . . . . . . . . . . 60

6.2 Ergebnis der Analyse . . . . . . . . . . . . . . . 61

$\begin{array}{ll}\text { A Glossar } & 63\end{array}$

B Feynman Diagramme der elastischen Streuung $\quad 64$

$\begin{array}{ll}\text { C Notationen } & 65\end{array}$

D Hodoskop 1 Daten $\quad 66$

$\begin{array}{ll}\text { Abbildungsverzeichnis } & 67\end{array}$

$\begin{array}{ll}\text { Tabellenverzeichnis } & 69\end{array}$

$\begin{array}{ll}\text { Literaturverzeichnis } & 70\end{array}$ 


\section{Einleitung}

Schon in der Antike beschäftigten sich die Griechischen Philosophen mit der Frage, woraus die Welt aufgebaut ist und so gilt Leukipp (5. Jh. v. Chr) als der Begründer der Atomlehre. Erst am Anfang des neunzehnten Jahrhunderts gelang es J. Dalton ${ }^{1}$ in den Jahren 1803 bis 1808 diese Theorie in ein wissenschaftliches Konzept zu münzen. Die von ihm entdeckten Gesetze der Erhaltung der Masse, der konstanten Proportionen und der multiplen Proportionen sind von grundlegender Bedeutung in der Chemie. Hierauf aufbauend gelang es D. Mandelejew ${ }^{2}$ im Jahr 1869 mit dem Periodensystem der Elemente Ordnung in die Welt der chemischen Elemente zu bringen. Doch schon bald machten neuere Experimente, wie M. Faradays Experimente zur Elektrolyse (18321833), sowie die von J. Plücker entdeckten Kathodenstrahlen (1859), es deutlich, dass die chemischen Elemente nicht ganz die átomos der Atomlehre sein konnten. Besonders die Experimente mit der Elektrizität zeigten, dass die Atome selbst noch aus kleineren Teilchen aufgebaut sein müssen. So war es G. J. Stoney, der die Existenz elektrischer Ladungsträger, die mit Atomen assoziiert sind, vorschlug und ihnen 1891 den Namen Elektron gab.

Die Entdeckung der Kanalstrahlen von E. Goldstein 1886 und die Bestimmung des Verhältnisses von $e / m$ für das Elektron (1897) sowie für das Proton durch J. Thomson 1906 bereiteten die Grundlage der genialen Deutung des berühmten Rutherfordschen Streuexperiments von 1911. E. Rutherford legte mit seinem Atommodell und der Vorhersage des Neutron (1920) die Grundlagen für die modernen Theorien des Atomaufbaus, welche zwanglos die Ordnung des Periodensystems erklärte. Doch die átomos als die elementaren Bausteine der Materie waren noch nicht gefunden.

Bevor J. Chadwick 1932 das Neutron experimentell Nachweisen konnte, postulierte W. Pauli ein weiteres Elementarteilchen, das Neutrino. Ein Teilchen das noch heute nichts von seiner Brisanz verloren hat. Damals eingeführt, um die Erhaltungssätze der Energie und des Impulses zu retten, dient es noch heute als Schlüssel zur tieferen Erkenntnis des grundlegenden Verständnisses des Aufbaus der Materie.

Mit der Entdeckung des Myons in der Kosmischen Strahlung 1936 durch C.D. Andersen begann eine Periode der stetigen Entdeckung von neuen Elementarteilchen, welche in den 50er und 60er Jahren durch die Beschleunigeranlagen eine neue Qualität erhielt. So stand man 1964 vor einem ganzen „Zoo" von über hundert Teilchen, von denen wohl kaum einer annahm, dass diese noch alle elementar sein könnten. Es waren M. Gell-Mann und G. Zweig die mit dem Quarkmodell (1964), das ursprünglich vorgeschlagen wurde, um eine Systematik in die Vielfalt der bis dahin entdeckten Hadronen zu bringen, ein Konzept präsentierte, das sich als weitaus tragfähiger erweisen sollte, als ursprünglich angenommen. Waren die drei postulierten Quarks up, down und strange anfangs nicht mehr als ein mathematisches Modell, so setzten sie sich mit der Erklärung des Skalenverhaltens der tiefinelastischen Streuung durch R.P. Feynman (Partonenmodell) weiter als physikalische Realität durch und spätestens seit der Entdeckung des $J / \psi(1974)$ ist das Quarkmodell allgemein akzeptiert.

Heutzutage wissen wir, daß es sechs Quarks gibt, aus denen die gesamte hadro-

\footnotetext{
${ }^{1}$ 1766-1844: Gesetz der Erhaltung der Masse, Gesetz der konstanten Proportionen und Gesetz der multiplen Proportionen

${ }^{2} 1834-1907$
} 
nische Materie aufgebaut ist. Die Quarks unterliegen als Träger der Farbladungen ${ }^{3}$ der starken Wechselwirkung und die Tatsache, dass Quarks in der Natur nicht einzeln beobachtet werden können, führt zur Hypothese des Farbeinschlusses (confinement), wonach Quarks nur in farbneutralen Zuständen vorkommen können. Eine der statischen Größen, welche direkt mit dem Confinement verknüpft ist und nebenbei auch eine sehr anschauliche Eigenschaften der zusammengesetzten Teilchen beschriebt, ist die Größe der Teilchen oder genauer der mittlere quadratische Ladungsradius der Teilchen.

\begin{tabular}{|ll|l|l|l|r|c|}
\hline & & $\mathrm{Q}$ & $\mathrm{I}\left(\mathrm{J}^{P}\right)$ & \multicolumn{1}{|c|}{$I_{3}$} & $\mathrm{~m}$ (Current Quark) & Quantenzahl \\
\hline \hline $\mathrm{d}$ & (down) & $-1 / 3$ & $\left.\frac{1}{2}^{+} \frac{1}{2}^{+}\right)$ & $+\frac{1}{2}$ & $3-9 \mathrm{MeV} / c^{2}$ & \\
$\mathrm{u}$ & (up) & $+2 / 3$ & $\frac{1}{2}^{+}\left(\frac{1}{2}^{+}\right)$ & $-\frac{1}{2}$ & $1.5-5 \mathrm{MeV} / c^{2}$ & \\
\hline $\mathrm{s}$ & (strange) & $-1 / 3$ & $0\left(\frac{1}{2}^{+}\right)$ & $-\frac{1}{2}$ & $60-170 \mathrm{MeV} / c^{2}$ & Strangeness =-1 \\
$\mathrm{c}$ & (charm) & $+2 / 3$ & $0\left(\frac{1}{2}^{+}\right)$ & $-\frac{1}{2}$ & $1.0-1.4 \mathrm{GeV} / c^{2}$ & Charm $=+1$ \\
\hline $\mathrm{b}$ & (bottom) & $-1 / 3$ & $0\left(\frac{1}{2}^{+}\right)$ & $-\frac{1}{2}$ & $4.1-4.4 \mathrm{GeV} / c^{2}$ & Bottom =-1 \\
$\mathrm{t}$ & (top ) & $+2 / 3$ & $0\left(\frac{1}{2}^{+}\right)$ & $-\frac{1}{2}$ & $174.3 \pm 5.1 \mathrm{GeV} / c^{2}$ & Top =+1 \\
\hline
\end{tabular}

Tabelle 1.1: Eigenschaften der Quarks[1].

Der elektromagnetische Ladungsradius des Protons wurde von R. Hofstädter [2] erstmals 1956 gemessen. Dabei wurden hochenergetische Elektronen an einem Wasserstofftarget gestreut und der Wirkungsquerschnitt als Funktion der Energie und des Streuwinkels gemessen. Diese Messung wurde später für verschieden Teilchen wiederholt. Um hierbei auch instabile Teilche vermessen zu können, wird das Meßprinzip dahingehend geändert, dass man das zu vermessende Teilchen auf quasi-ruhende Elektronen in der Hülle eines Targetmaterials schießt.

Dabei ergibt es sich, dass der Ladungsradius von Kaonen $(\bar{u} s)\left\langle r_{K}^{2}\right\rangle=0.35 \pm$ $0.05 \mathrm{fm}^{2}$ [3] kleiner ist als der von Pionen $(\bar{u} d)\left\langle r_{\pi}^{2}\right\rangle=0.44 \pm 0.01 \mathrm{fm}^{2}$ [4], welches im Konstituentenquark-Modell qualitativ erklärbar ist, da sich in diesem Modell der Radius eines Teilchens aus den Radien seiner Substituenten ergibt und deren Größe skaliert umgekehrt proportional zu deren Masse. Folglich sollte das Kaon, welches eine strange-Quark beinhaltet, auch einen kleineren Radius aufweisen. Dabei beträgt der Radiusunterschied zwischen diesen beiden Mesonen etwa $\left\langle r_{\pi}^{2}\right\rangle-\left\langle r_{K}^{2}\right\rangle \approx 0.1 \mathrm{fm}^{2}$.

Ob Baryonen, welche aus drei Quark aufgebaut sind, ein ähnliches Verhalten zeigen wie die Mesonen, muß noch abschließend geklärt werden. Im Rahmen des SELEX Experiments arbeitet das Max-Planck-Institut für Kernphysik an dieser Aufgabe.

\footnotetext{
${ }^{3}$ Die Farbladung kann drei Werte annehmen, welche üblicherweise mit rot, grün und blau bezeichnet werden
} 


\section{Elastische Pion-Elektron-Streuung}

Bei der Streuung von hochenergetischen Pionen an ruhenden Elektronen handelt es sich um die Streuung eines räumlich ausgedehnten Spin-0-Teilchens an einem punktförmigen Spin- $\frac{1}{2}$-Teilchen. Die Betrachtung der Kinematik ergibt ein Maß für den möglichen Energieübertrag, während die Untersuchung des Wirkungsquerschnitts Einsicht in die elektromagnetische Struktur des ausgedehnten Teilchens gibt. Schließlich betrachten wir noch jene inelastischen Pion-Elektron-Streuungen, welche experimentell aufgrund endlicher Auflösungen nicht von elastischen unterschieden werden können.

\subsection{Elastische Streuung}

Der Eingangskanal der Pion-Elektron-Streuung im Laborsystem (Abb. 2.1) ist durch das einfliegende Pion und das ruhende Elektron bestimmt, während sich im Ausgangskanal beide Teilchen in Bewegung befinden.

$$
\begin{array}{ll}
p_{\pi}=\left(E_{\pi}, \vec{p}_{\pi}\right) & p_{e}=\left(m_{e}, 0\right) \\
p_{\pi}^{\prime}=\left(E_{\pi}^{\prime}, \vec{p}_{\pi}^{\prime}\right) & p_{e}^{\prime}=\left(E_{e}^{\prime}, \vec{p}_{e}^{\prime}\right)
\end{array}
$$

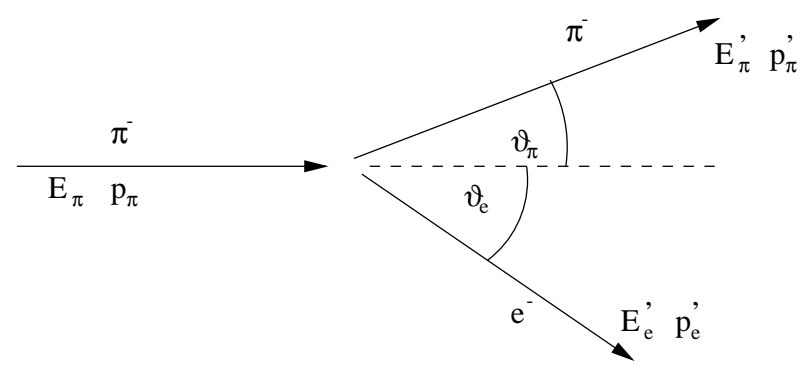

Abbildung 2.1: Kinematik der Pion-Elektron-Streuung im Laborsystem.

Der Viererimpulsübertrag, dessen Quadrat eine lorentzinvariante Größe ist, wird definiert über

$$
\mathrm{Q}^{2}=-t=-\left(p_{e}-p_{e}^{\prime}\right)^{2}=\left(p_{\pi}-p_{\pi}^{\prime}\right)^{2} .
$$

Aus der Erhaltung des Viererimpulses

$$
p_{\pi}+p_{e}=p_{\pi}^{\prime}+p_{e}^{\prime}
$$

ergibt sich mit

$$
p_{\pi} p_{e}=p_{\pi}^{\prime} p_{e}^{\prime}=p_{\pi}^{\prime}\left(p_{\pi}+p_{e}-p_{\pi}^{\prime}\right)=E_{\pi} E_{\pi}^{\prime}-\vec{p}_{\pi} \vec{p}_{\pi}^{\prime}+E_{\pi}^{\prime} m_{e}-m_{\pi}^{2}
$$

für die Streuwinkel des Pions $\vartheta_{\pi}$ und des Elektrons $\vartheta_{e}$

$$
\begin{aligned}
\cos \vartheta_{\pi} & =\frac{E_{\pi}^{\prime}\left(m_{e}+E_{\pi}\right)-m_{e} E_{\pi}-m_{\pi}^{2}}{\left|\vec{p}_{\pi}\right|\left|\vec{p}_{\pi}^{\prime}\right|} \\
\cos \vartheta_{e} & =\frac{E_{e}^{\prime}\left(m_{e}+E_{\pi}\right)-m_{e} E_{\pi}-m_{e}^{2}}{\left|\vec{p}_{\pi}\right|\left|\vec{p}_{e}^{\prime}\right|} .
\end{aligned}
$$


Das Quadrat des Viererimpulsübertrages $Q^{2}$ kann aus verschiedenen Observablen errechnet werden:

1. Aus dem Energiegewinn des gestreuten Elektrons:

$$
\mathrm{Q}^{2}=2 m_{e}\left(E_{e}^{\prime}-m_{e}\right) \approx 2 m_{e} E_{e}^{\prime}
$$

2. Aus dem Energieverlust des gestreuten Pions:

$$
\mathrm{Q}^{2}=2 m_{e}\left(E_{\pi}-E_{\pi}^{\prime}\right)
$$

3. Aus dem Elektronwinkel $\vartheta_{e}$ und dem Impuls des einlaufenden Pions $p_{\pi}$ :

$$
\mathrm{Q}^{2}=\frac{4 m_{e}^{2}\left(p_{\pi} \cos \vartheta_{e}\right)^{2}}{\left(E_{\pi}+m_{e}\right)^{2}-\left(p_{\pi} \cos \vartheta_{e}\right)^{2}}
$$

4. Aus dem Pionenwinkel $\vartheta_{\pi}$ und dem Impuls des einlaufenden Pions $p_{\pi}$ :

$$
\mathrm{Q}^{2}=\frac{2 p_{\pi} m_{e}\left(p_{\pi}-p_{\pi}^{\prime}\right) \cos \vartheta_{\pi}}{\left(E_{\pi}+m_{e}\right)}
$$

Folglich ist $\mathrm{Q}^{2}$ überbestimmt und kann aus einem Constrained Non-Linear Least Square Fit (vgl. Abschn. 5.2.3 mehrerer Observablen gewonnen werden. Andererseits lassen sich obige Relationen auch nutzen, um die Konsistenz verschiedener Observablen zu studieren.

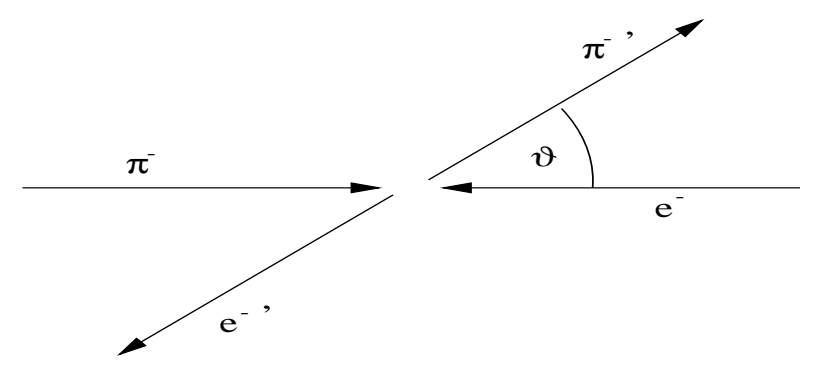

Abbildung 2.2: Kinematik der Pion-Elektron-Streuung im Schwerpunktsystem.

Betrachtet man die Streuung im Schwerpunktsystem (Abb. 2.2), so ergibt sich für das Maximum des Quadrats des Viererimpulsübertrages bei $\vartheta=180^{\circ}$ aus $\vec{p}_{e}=-\vec{p}_{e}^{\prime}$

$$
\mathrm{Q}_{\text {max }}^{2}=-\left(\begin{array}{c}
E_{e}-E_{e}^{\prime} \\
\vec{p}_{e}-\vec{p}_{e}^{\prime}
\end{array}\right)_{c m s}^{2}=4 \vec{p}_{c m s}^{2}
$$

mit $\left|\vec{p}_{e}\right|=\left|\vec{p}_{c m s}\right|$. Aus der Schwerpunktenergie $s$ im Schwerpunktsystem erhält man $\operatorname{mit} \vec{p}_{e}=-\vec{p}_{\pi}$ und $E_{\pi}^{2}=E_{e}^{2}+m_{\pi}^{2}-m_{e}^{2}$

$$
\begin{aligned}
s & =\left(\begin{array}{c}
E_{e}+E_{\pi} \\
\vec{p}_{e}+\vec{p}_{\pi}
\end{array}\right)_{c m s}^{2}=E_{e}^{2}+E_{\pi}^{2}+2 E_{e} E_{\pi} \\
& =m_{\pi}^{2}-m_{e}^{2}+2 E_{e}\left(E_{e}+E_{\pi}\right) \\
2 E_{e} & =\frac{s-m_{\pi}^{2}+m_{e}^{2}}{\sqrt{s}}
\end{aligned}
$$


Tabelle 2.1: $\mathrm{Q}_{\max }^{2}$ für verschiedene Strahlteilchen im SELEX Experiment, getrennt nach Ladung und für die jeweiligen mittleren Strahlimpulse.

\begin{tabular}{|c|c|c|}
\hline $\begin{array}{c}\text { Strahl- } \\
\text { teilchen }\end{array}$ & $\begin{array}{c}\text { Impuls } \\
{[\mathrm{GeV} / \mathrm{c}]}\end{array}$ & $\begin{array}{c}\mathrm{Q}_{\text {max }}^{2} \\
{\left[\mathrm{GeV}^{2} / c^{2}\right]}\end{array}$ \\
\hline \hline$\pi^{-}$ & & 0.595 \\
$\Sigma^{-}$ & 600 & $\begin{array}{l}0.184 \\
0.159\end{array}$ \\
$\Xi^{-}$ & & 0.219 \\
\hline \hline$p$ & 550 & 0.160 \\
$\Sigma^{+}$ & & \multicolumn{2}{|c}{} \\
\hline
\end{tabular}

und damit aus $E_{e}^{2}=m_{e}^{2}+\vec{p}_{c m s}^{2}$ und 2.12:

$$
\mathrm{Q}_{\text {max }}^{2}=\frac{\left(s-m_{\pi}^{2}+m_{e}^{2}\right)^{2}}{s}-4 m_{e}^{2}
$$

Rechnet man dies in das Laborsystem um, in dem das Elektron als ruhend angenommen sei, so ergibt sich aus $s=m_{e}^{2}+m_{\pi}^{2}+2 m_{e} E_{\pi}$

$$
\mathrm{Q}_{\max }^{2}=\frac{4 m_{e}^{2}\left(E_{\pi}^{2}-m_{\pi}^{2}\right)}{m_{e}^{2}+m_{\pi}^{2}+2 m_{e} E_{\pi}}
$$

Tabelle 2.1 listet $\mathrm{Q}_{\text {max }}^{2}$ für die verschiedenen, in SELEX untersuchten Strahlteilchen auf und in Abb. 2.3 zeigt das Verhalten der Streuwinkel als Funktion des Impulsübertrages und die Winkel-Winkel-Relation für verschiedene Impulse des einlaufenden Pions.

\subsection{Formfaktoren und Ladungsradius}

Berechnet man in der Born'schen Näherung den Wirkungsquerschnitt für die Streuung relativistischer Elektronen an einer räumlich ausgedehnten Ladungsverteilung $\rho(\vec{x})$ [5], so erhält man den Mott-Wirkungsquerschnitt ohne Rückstoßterm, multipliziert mit dem Absolutbetrag eines Formfaktors $F(\vec{q})$

$$
\left(\frac{\mathrm{d} \sigma}{\mathrm{d} \Omega}\right)_{\exp }=\left(\frac{\mathrm{d} \sigma}{\mathrm{d} \Omega}\right)_{\mathrm{Mott}}^{*} \cdot|F(\vec{q})|^{2}
$$

mit

$$
\left(\frac{\mathrm{d} \sigma}{\mathrm{d} \Omega}\right)_{\mathrm{Mott}}^{*}=\frac{4 Z^{4} \alpha^{2}(\hbar c)^{2} E^{2}}{\vec{q}^{4} c^{4}} \cdot \cos ^{2} \frac{\theta}{2}
$$

Der Formfaktor $F$ ist eine Funktion des Impulsübertrages $\vec{q}$ und für den einfachsten Fall der Born'schen Näherung (ohne Rückstoß) gleich der Fouriertransformierten der Ladungsverteilung $\rho(\vec{x})$.

$$
F(\vec{q})=\int e^{i \vec{q} \vec{x} / \hbar} \rho(\vec{x}) \mathrm{d}^{3} x
$$



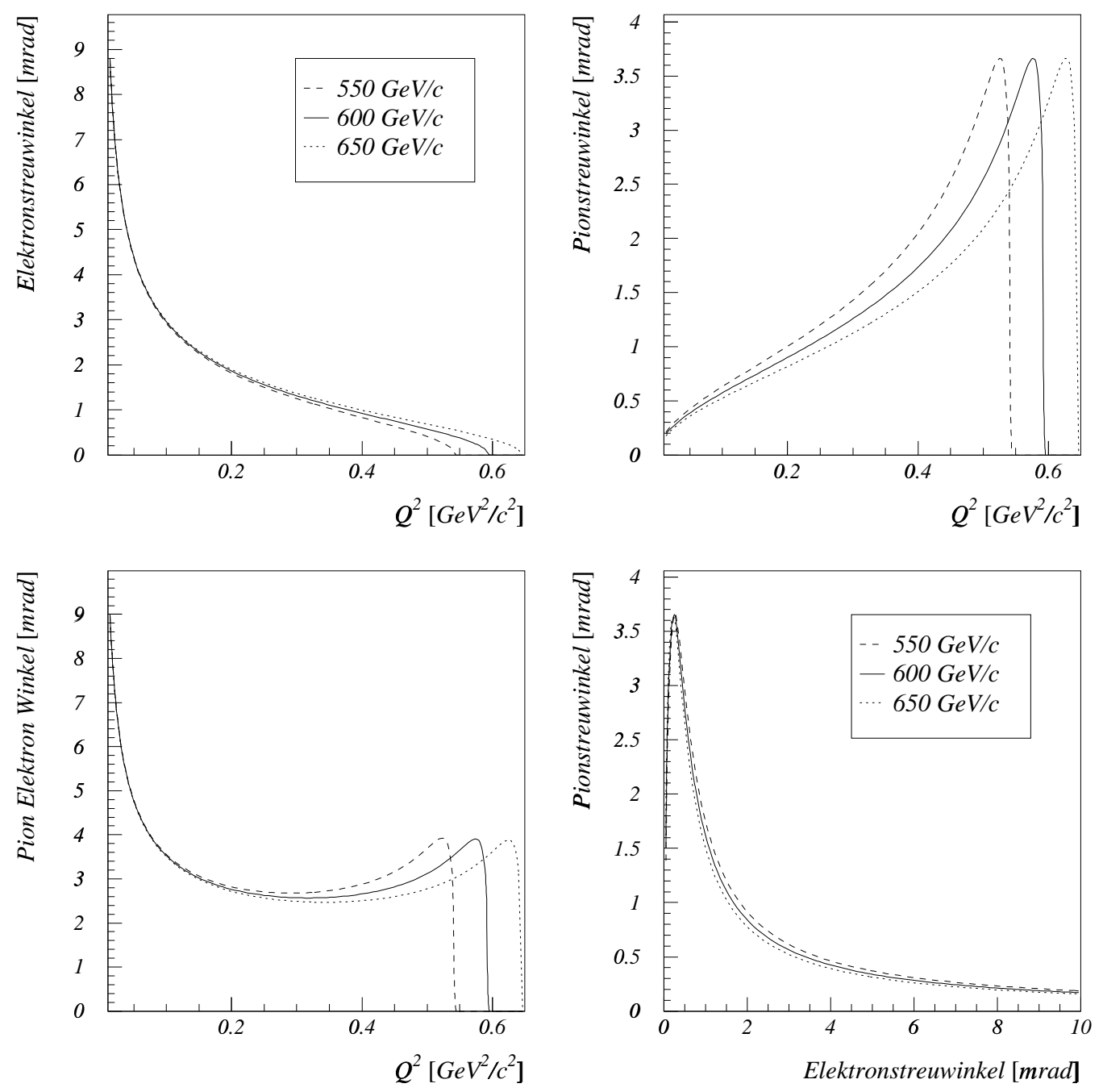

Abbildung 2.3: $\quad \mathrm{Q}^{2}$-Abhängigkeit verschiedener Observablen der Pion-ElektronStreuung bei verschiedenen Strahlimpulsen. Oben links der Elektronenstreuwinkel, rechts der Pionenstreuwinkel, unten links der Pion-Elektron-Öffnungswinkel und rechts die Winkel-Winkel-Korrelation.

Legt man eine kugelsymmetrische Ladungsverteilung zu Grunde und entwickelt die Exponentialfunktion für kleine $\vec{q} \vec{x}$ um $|\vec{q}|=0$ herum, so erhält man

$$
\begin{aligned}
F(|\vec{q}|) & =\int e^{i \vec{q} \vec{x} / \hbar} \rho(\vec{x}) \mathrm{d}^{3} x \\
& =\int\left(1+\frac{i \vec{q} \vec{x}}{\hbar}-\frac{(\vec{q} \vec{x})^{2}}{2 \hbar^{2}}+\ldots\right) \rho(x) \mathrm{d}^{3} x \\
& =1-\frac{|\vec{q}|^{2}}{6 \hbar^{2}} \int x^{2} \rho(x) \mathrm{d}^{3} x+\mathcal{O}\left(\vec{q}^{4}\right) \\
& =1-\frac{|\vec{q}|^{2}}{6 \hbar^{2}}\left\langle r^{2}\right\rangle+\mathcal{O}\left(\vec{q}^{4}\right)
\end{aligned}
$$


mit dem zweiten Moment der Ladungsverteilung, welche normiert sei, als mittleren quadratischen Ladungsradius

$$
\left\langle r^{2}\right\rangle=-\left.6 \hbar^{2} \frac{\mathrm{d} F(|\vec{q}|)}{\mathrm{d} \vec{q}^{2}}\right|_{|\vec{q}| \rightarrow 0}=4 \pi \int r^{2} \rho(r) \mathrm{d} r
$$

Bei der Pion-Elektron-Streuung verwendet man die inverse Kinematik, d.h. das Pion wird an einem Hüllenelektron gestreut. Da die Geschwindigkeit des Pions mit der Energie von $600 \mathrm{GeV}$ um ein Vielfaches größer ist, als die charakteristische Geschwindigkeit von Elektronen in der Atomhülle, kann das Elektron in der Beschreibung des Streuprozesses als ruhend angenommen werden.

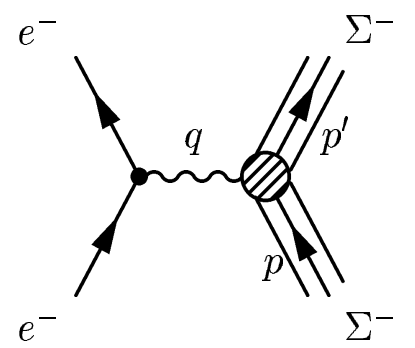

Abbildung 2.4: Feynmandiagramm 1. Ordnung der elastischen Sigma-ElektronStreuung als Beispiel für die Streuung zweier Spin- $\frac{1}{2}$-Teilchen.

Behandelt man die elastische Streuung zweier Spin- $\frac{1}{2}$-Teilchen im allgemeinsten Fall, so müssen auch Spineffekte und anormale magnetische Momente $\kappa$ berücksichtigt werden, wie dieses z.B. bei der Hyperon-Elektron-Streuung der Fall ist. Hierbei berechnet man nach den Regeln für Feynmangraphen das Matrixelement der Streumatrix zu Abb. 2.4. Den Übergangsstrom des Hyperons berechnet man aus den bilinearen Kovarianten der Dirac-Theorie (siehe z.B. [6]). Aus den Einschränkungen der Hermitezität und der Eichinvarianz (Strominvarianz) folgt, dass der Übergangsstrom, dargestellt durch den Diracspinor $u$ und die Diracmatrizen $\gamma_{\mu}$, in der Gordon-Zerlegung die Form

$$
\bar{u}\left(p^{\prime}\right)\left(\gamma_{\mu} F_{1}\left(q^{2}\right)+i \frac{\kappa}{2 M} F_{2}\left(q^{2}\right) q^{\nu} \sigma_{\mu \nu}\right) u(p)
$$

annimmt. Die reellen Formfaktoren $F_{1}$ und $F_{2}$ können nur von dem Quadrat des Impulsübertrags $q^{2}$ abhängen, da dies der einzige, sich ändernde Lorentz-Skalar ist, der in der elastischen Hyperon-Elektron-Streuung zur Verfügung steht, und weil sich das Hyperon vor und nach der Streuung auf der Massenschale befindet.

Die Bedeutung der Formfaktoren erschließt sich aus der Betrachtung eines nichtrelativistischen Hyperons in einem äußeren elektromagnetischen Feld. Der Formfaktor $F_{1}$ beschreibt die Energie in einem äußeren elektrischen Feld, während die Energie eines Spin- $\frac{1}{2}$-Teilchens mit dem $g$-Faktor (gyromagnetischer Faktor) $g=2\left(1+F_{2}(0)\right)$ im homogenen Magnetfeld von $F_{2}$ bestimmt wird und dieser also das anomale magnetische Moment des Teilchens beschreibt [7].

Setzt man diesen Hyperonenstrom in das Matrixelement ein und berechnet damit den differentiellen Wirkungsquerschnitt, wobei über die Spinzustände der einlaufenden Teilchen gemittelt wird und die der auslaufenden summiert werden, da keine Polarisation gemessen wird, so ergibt sich unter Vernachlässigung der Elektronenmasse $m_{e}$ die 
lorentzinvariante Rosenbluth-Formel [8]

$$
\begin{aligned}
\frac{\mathrm{d} \sigma}{\mathrm{d} t}= & \frac{4 \pi \alpha^{2}}{t^{2}} \cdot \frac{s t+\left(s-M^{2}\right)^{2}}{\left(s-M^{2}\right)^{2}} \\
& \cdot\left[F_{1}^{2}-\frac{t}{4 M^{2}} \kappa^{2} F_{2}^{2}+\frac{t^{2}}{2\left(s t+\left(s-M^{2}\right)^{2}\right)}\left(F_{1}+\kappa F_{2}\right)^{2}\right]
\end{aligned}
$$

in den Mandelstamvariablen $s$ und $t$ (Gl. 2.13 und 2.3), sowie in natürlichen Einheiten mit $c=\hbar=1$. Der zweite Faktor läßt sich mit Gl. 2.17 auf die einfache Form

$$
\frac{s t+\left(s-M^{2}\right)^{2}}{\left(s-M^{2}\right)^{2}}=1-\frac{\mathrm{Q}^{2}}{\mathrm{Q}_{\max }^{2}}
$$

bringen. Faßt man die beiden Strukturfunktionen zu einer neuen Funktion $\hat{F}\left(\mathrm{Q}^{2}\right)$

$$
\hat{F}^{2}=F_{1}^{2}+\frac{\mathrm{Q}^{2} \kappa^{2}}{4 M^{2}} F_{2}^{2}+\frac{Q^{4}}{8 m_{e}^{2} E^{2}-2 \mathrm{Q}^{2}\left(M^{2}+2 m_{e} E\right)}\left(F_{1}+\kappa F_{2}\right)^{2}
$$

zusammen, so erhält man einen einfachen Ausdruck für die Rosenbluth-Formel

$$
\frac{\mathrm{d} \sigma}{\mathrm{dQ}^{2}}=\left(\frac{4 \pi \alpha^{2}}{Q^{4}}\left(1-\frac{\mathrm{Q}^{2}}{\mathrm{Q}_{\max }^{2}}\right)\right)_{M o t t} \hat{F}^{2}\left(\mathrm{Q}^{2}\right)
$$

Die beiden Formfaktoren $F_{1}$ und $F_{2}$ der Rosenbluth-Formel lassen sich entkoppeln, indem man die beiden Sachs'schen Formfaktoren $G_{E}$ und $G_{M}$ einführt [9]

$$
\begin{aligned}
G_{E} & :=F_{1}-\frac{\mathrm{Q}^{2} \kappa}{4 m_{\pi}^{2}} F_{2} \\
G_{M} & :=F_{1}+\kappa F_{2}
\end{aligned}
$$

Mit diesen Variablen verschwinden die Kreuzterme von $F_{1}$ und $F_{2}$ aus der RosenbluthFormel. Man bezeichnet die Sachs'schen Formfaktoren auch als elektrischen und magnetischen Formfaktor, da bei geeigneter Wahl des Koordinatensystems, dem sogenannten Breit-Bezugssystem, in dem keine Energie übertragen wird, die beiden Formfaktoren in die Fouriertransformierten der Ladungs- und Stromverteilung übergehen.

Entsprechend dem mittleren quadratischen Ladungsradius definiert man die mittleren Sachs'schen Ladungsradien für die Verteilungen der elektrischen Ladungen $\left\langle r_{E}^{2}\right\rangle$ und der magnetischen Dipole $\left\langle r_{M}^{2}\right\rangle$ und kann diese über die Ableitungen der Formfaktoren messen. Die Formfaktoren werden hierzu bei kleinen Viererimpulsüberträgen $\mathrm{Q}^{2}$ gemessen und nach $\mathrm{Q}^{2}=0$ extrapoliert.

$$
\begin{aligned}
\left\langle r_{E}^{2}\right\rangle & =-\left.6 \frac{1}{G_{E}(0)} \cdot \frac{\mathrm{d} G_{E}\left(\mathrm{Q}^{2}\right)}{\mathrm{dQ}^{2}}\right|_{\mathrm{Q}^{2}=0} \\
\left\langle r_{M}^{2}\right\rangle & =-\left.6 \frac{1}{G_{M}(0)} \cdot \frac{\mathrm{d} G_{M}\left(\mathrm{Q}^{2}\right)}{\mathrm{dQ}^{2}}\right|_{\mathrm{Q}^{2}=0}
\end{aligned}
$$

Die $\mathrm{Q}^{2}$-Abhängigkeit der Formfaktoren läßt sich für Baryonen recht gut durch einen Dipolfit approximieren:

$$
\begin{aligned}
G_{E}\left(\mathrm{Q}^{2}\right) & =\left(1+\frac{\mathrm{Q}^{2}}{\Lambda^{2}}\right)^{-2} \approx 1-\frac{2 \mathrm{Q}^{2}}{\Lambda^{2}} \\
G_{M}\left(\mathrm{Q}^{2}\right) & =(\kappa-1) \cdot\left(1+\frac{\mathrm{Q}^{2}}{\Lambda^{2}}\right)^{-2}=\mu \cdot G_{E}\left(\mathrm{Q}^{2}\right)
\end{aligned}
$$


mit dem normalen magnetischen Moment $\mu=\kappa-1$.

Für Spin-0-Teilchen vereinfacht sich die ganze Beschreibung, da hier keine anomales magnetische Moment berücksichtigt werden muß. Entsprechend folgt für den Wirkungsquerschnitt

$$
\frac{\mathrm{d} \sigma}{\mathrm{dQ}^{2}}=\left(\frac{4 \pi \alpha^{2}}{Q^{4}}\left(1-\frac{\mathrm{Q}^{2}}{\mathrm{Q}_{\max }^{2}}\right)\right)_{M o t t} \cdot F^{2}\left(\mathrm{Q}^{2}\right)
$$

mit dem Formfaktor $F^{2}\left(\mathrm{Q}^{2}\right)$. Der Formfaktor $F^{2}\left(\mathrm{Q}^{2}\right)$ sich durch einen Monopolfit beschreiben läßt

$$
F\left(\mathrm{Q}^{2}\right)=\left(1+\frac{\mathrm{Q}^{2}}{\Lambda^{2}}\right)^{-1} \approx 1-\frac{\mathrm{Q}^{2}}{\Lambda^{2}} .
$$

Aus diesen folgt der mittlere quadratische Ladungsradius über

$$
\left\langle r^{2}\right\rangle=-\left.6 \frac{\mathrm{d} F\left(\mathrm{Q}^{2}\right)}{\mathrm{dQ}^{2}}\right|_{\mathrm{Q}^{2}=0} .
$$

Der Wirkungsquerschnitt und der Formfaktor sind in Abb. 2.5 gezeigt.

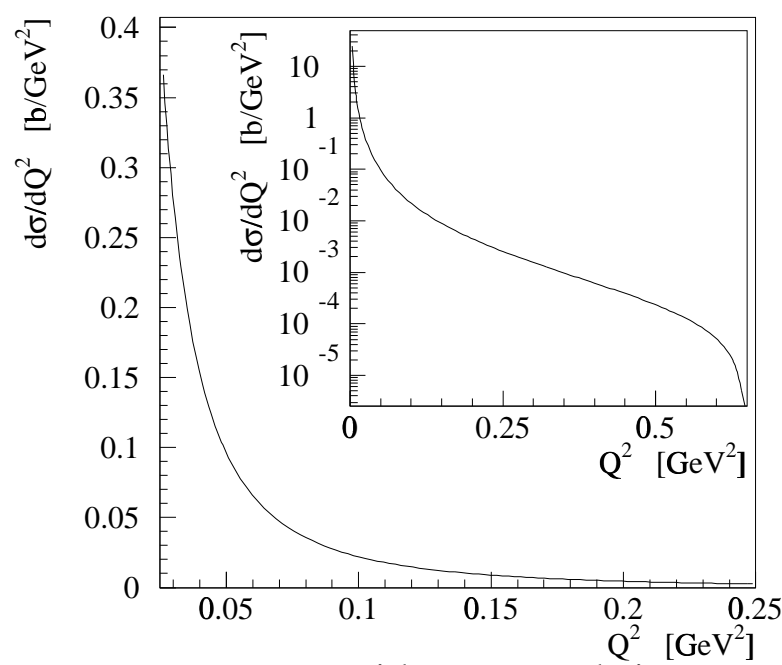

Mott-Wirkungsquerschnitt

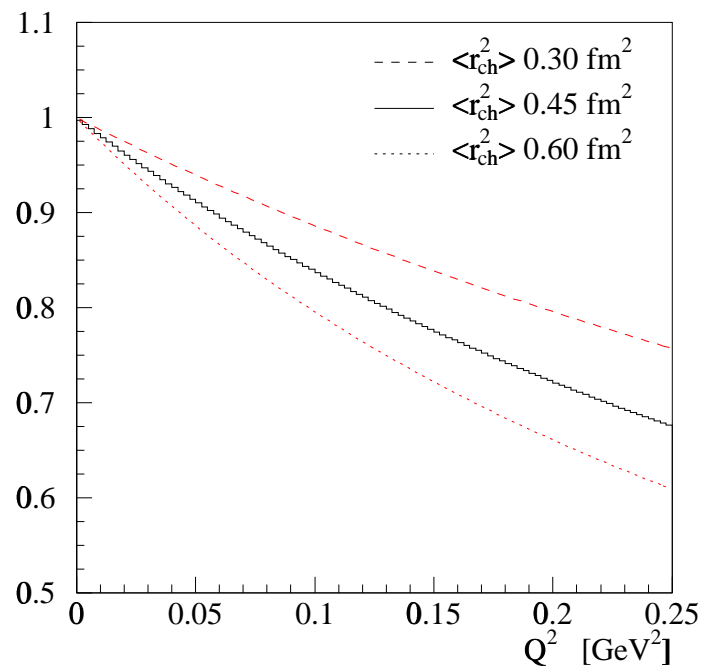

Formfaktor

Abbildung 2.5: Mott-Wirkungsquerschnitt für Pionen mit einer Energie von $650 \mathrm{GeV}$ (links) und der Formfaktor für verschiedene Radien (rechts).

\subsection{Strahlungskorrekturen}

Das bei der Herleitung der Rosenbluth-Formel (Gl. 2.23) im Kapitel 2.2 verwendete Modell der Born'schen Näherung, beschreibt einen Ein-Photonen-Austausch (Abb. 2.6). Unter dem Begriff Strahlungskorrekturen werden sowohl die elastischen Prozesse höherer Ordnung zusammengefaßt, als auch all jene inelastischen Prozesse, welche auf Grund endlicher Detektorauflösung nicht von den untersuchten Prozessen unterscheidbar sind. Da die elastische und die inelastische Streuung unterschiedliche Ausgangskanäle haben, kann es keine Interferenzen zwischen diesen beiden geben und der beobachtete Wirkungsquerschnitt ist gleich der Summe des elastischen und des inelastischen Wirkungsquerschnitts. 


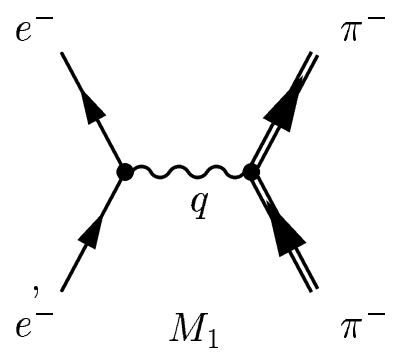

Abbildung 2.6: Feynmandiagramm 1. Ordnung der Born'schen Näherung der elastischen Pion-Elektron-Streuung.

Elastische Prozesse höherer Ordnung: Als Prozesse höherer Ordnung bei der elastischen Streuung ist es ausreichend, die zweite Ordnung mit zu betrachten, da jede weitere Ordnung mit dem Faktor $\alpha^{2}$ skaliert. Die elastischen Prozesse zweiter Ordnung sind die Selbstenergie-Diagramme, die Vakuumpolarisation und die Vertexkorrekturen (Abb. B.2), sowie Zwei-Photonen-Austausch-Diagramme (Abb. B.3).

Im folgenden wird die Vorgehensweise bei der Berechnung der Strahlungskorrekturen kurz skizziert, wobei sich diese an dem Paper von Kahane [10] orientiert. Für den elastischen Beitrag zum Wirkungsquerschnitt gilt

$$
\sigma \propto \sum_{\text {Spins }} M^{\dagger} M
$$

Wobei das Matrixelement $M$ die Summe aus den Matrixelementen $M_{i}$ (Abb. B.2 und B.3) ist,

$$
M=M_{1}+\sum_{i=2}^{11} M_{i}
$$

Beschränkt man sich auf die Ordnungen bis $\alpha^{3}$, so läßt sich zeigen [10], dass gilt

$$
M^{\dagger} M=M_{1}^{\dagger} M_{1}+2 \operatorname{Re} \sum_{i=2}^{11} M_{1}^{\dagger} M_{i}
$$

Der Beitrag $M_{1}^{\dagger} M_{1}$ liefert den Anteil der 1. Born'schen Näherung und die verbleibenden Terme sind Strahlungskorrekturen bis zur Ordnung $\alpha^{3}$. Die Matrixelemente $M_{2}-$ $M_{11}$ sind divergent für große Impulse der virtuellen Photonen (Infrarot-Divergenz). Diese Divergenzen lassen sich mit der Massen- und Ladungsrenormalisierung beseitigen $[10,11,12]$. Hierbei wird entweder ein Abschneideparameter verwendet oder eine fiktive Photonenmasse eingeführt. Dabei lassen sich die Matrixelemente $M_{2}-M_{8}$ bis auf einen multiplikativen Faktor $\delta_{1}$ auf $M_{1}$ zurückführen. Die Matrixelemente der Zwei-Photonen-Austausch Prozessen lassen sich auf diese Weise nicht direkt reduzieren. Deren Matrixelemente zerlegt man in einen nichtdivergenten Teil $\delta_{2}$ und einen die Infrarot-Divergenzen enthaltenden Teil $\delta_{\lambda}$.

Damit erhält man unter Vernachlässigung von $m_{e} / E_{e}^{\prime}$ gegenüber 1 für den Wechselwirkungsquerschnitt

$$
\left(\frac{\mathrm{d} \sigma_{e l}}{\mathrm{dQ}^{2}}\right)_{\text {elast }}=\left[1+\frac{\alpha}{\pi}\left(\delta_{1}+\delta_{2}\right)+\delta_{\lambda}\right] \cdot\left(\frac{\mathrm{d} \sigma}{\mathrm{dQ}^{2}}\right)_{B o r n}
$$




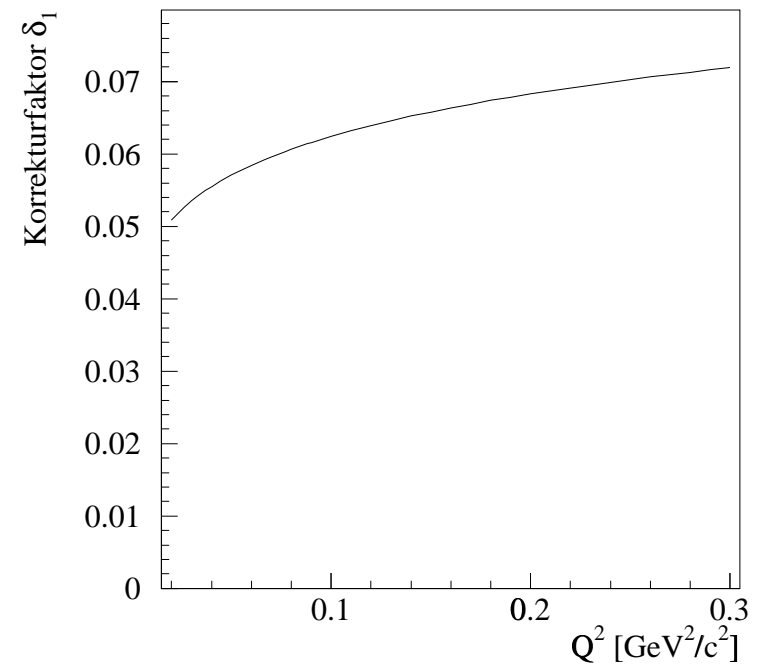

Korrektur $\delta_{1}$

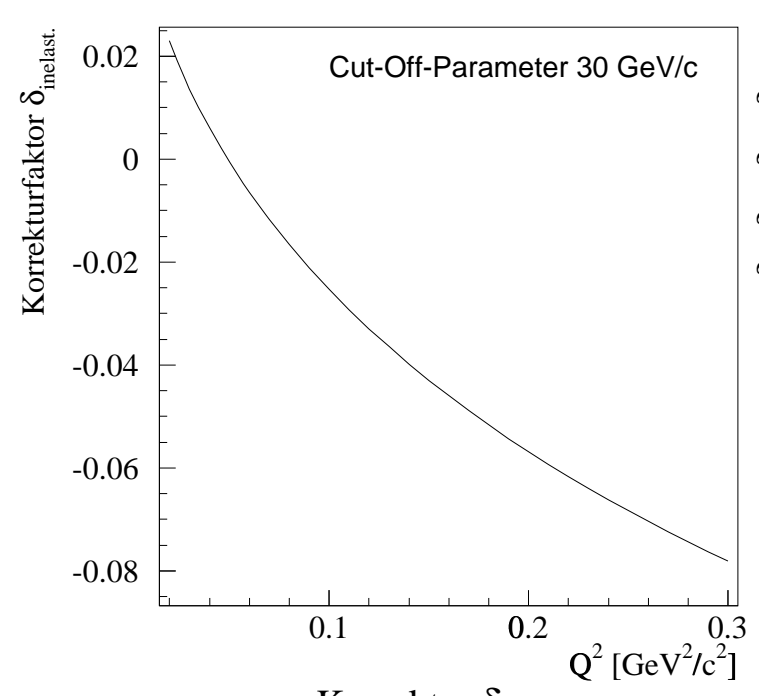

Korrektur $\delta_{\text {inelast. }}$

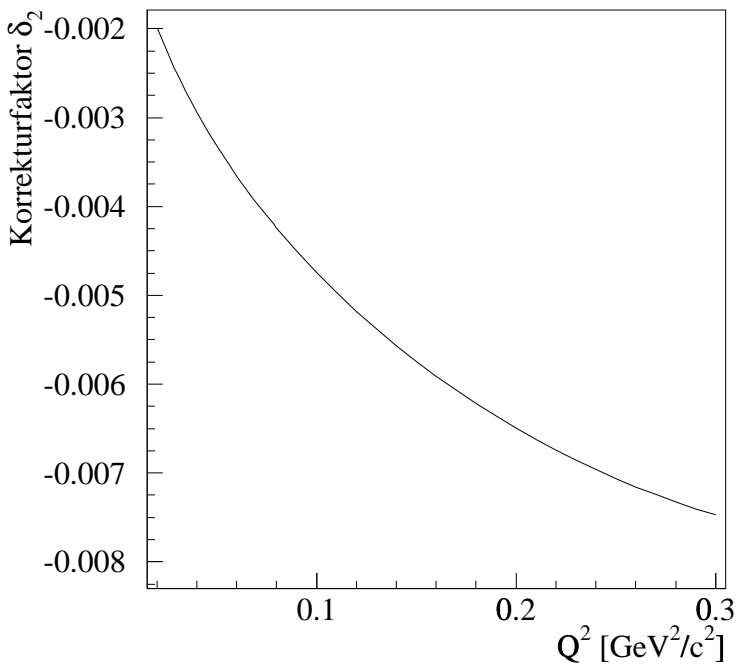

Korrektur $\delta_{2}$

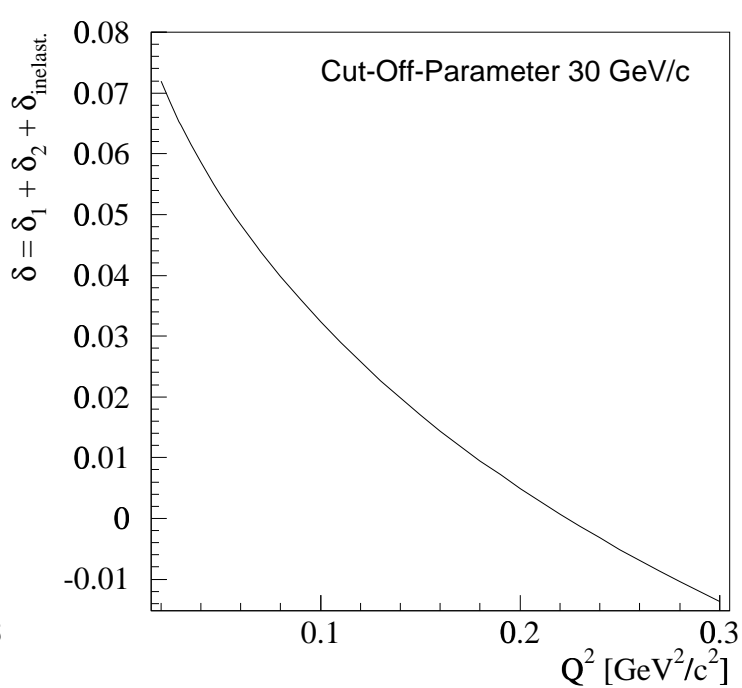

Gesamt-Strahlungskorrektur

Abbildung 2.7: Beiträge der einzelnen Strahlungskorrekturen aus den Vertexkorrekturen, der Vakuumpolarisation und den Selbstenergien des Elektrons und des Pions ( $\delta_{1}$ oben links), der Zwei-Photonen-Austausch-Wechselwirkung ( $\delta_{2}$ oben rechts) und der Beitrag der inelastischen Korrekturen aus den internen Bremsstrahlungsprozessen, berechnet mit einem Cut-Off-Parameter von $30 \mathrm{GeV} / c\left(\delta_{\text {inelast }}\right.$ unten links), sowie die summierte Gesamt-Strahlungskorrektur unten rechts.

mit den Beiträgen der Selbstenergie-, Vakuumpolarisations- und Vertexkorrekturdiagramme in $\delta_{1}$ und den Beiträgen der Zwei-Photonen-Austausch-Diagramme in $\delta_{2}$. Der Term $\delta_{\lambda}$, beinhaltet Terme aus den Zwei-Photonen-Austausch-Diagrammen, die Divergenzen enthalten, welche sich mit divergenten Termen aus den inelastischen Prozessen aufheben. Diese inelastische Prozesse müssen aufgrund endlicher Detektorauflösung immer berücksichtigt werden. 
Inelastische Prozesse erster Ordnung: Die inelastischen Beiträge zu den Strahlungskorrekturen stammen von der internen Bremsstrahlung des Elektrons und Pions (Abb. B.4) Sie hängen stark von experimentellen Gegebenheiten ab. Ein Kenngröße ist hierbei die minimale Energie die Photonen haben müssen, um detektiert werden zu können. Diese minimale Energie liegt bei SELEX bei $5 \mathrm{GeV}$, da aber die entsprechenden elektromagnetischen Kalorimeter in der Datenanalyse nicht genutzt werden, wurden die Strahlungskorrekturen mit einer Cut-Off-Parameter von $30 \mathrm{GeV} / c$ berechnet.

Der Beitrag der inelastischen Prozesse liefert zwei weitere Faktoren $\delta_{\text {inelast. }}$ und $\delta_{\lambda}^{\prime}$ zum Wechselwirkungsquerschnitt. Der Beitrag $\delta_{\lambda}^{\prime}$ enthält die selben Divergenzen wie der Beitrag $\delta_{\lambda}$ aus den Zwei-Photonen-Austausch-Diagrammen und kürzt sich mit diesem weg. Die Strahlungskorrekturen bis zur Ordnung $\alpha^{3}$ ergeben damit eine Änderung des Born'schen Wirkungsquerschnittes von der Form

$$
\left(\frac{\mathrm{d} \sigma}{\mathrm{d} \Omega}\right)_{\text {meas. }}=\left[1+\frac{\alpha}{\pi}\left(\delta_{1}+\delta_{2}+\delta_{\text {inelast }}\right)\right]\left(\frac{\mathrm{d} \sigma}{\mathrm{d} \Omega}\right)_{\text {Born }} .
$$

Die Beiträge dieser einzelnen Korrekturen sind in Abb. 2.7 dargestellt.

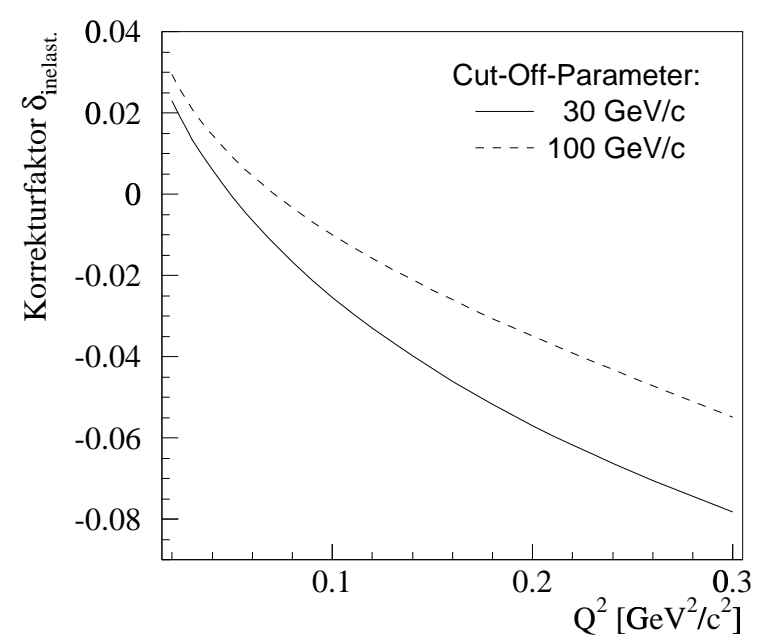

Korrektur $\delta_{\text {inelast. }}$

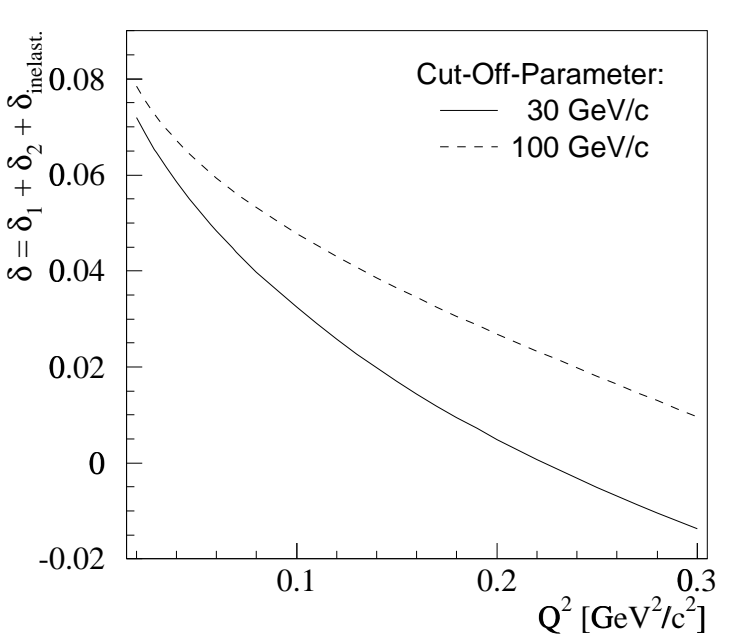

Gesamt-Strahlungskorrektur

Abbildung 2.8: Strahlungskorrekturen für einen Cut-Off-Parameter von $100 \mathrm{GeV} / c$. Die elastischen Beiträge entsprechen denen aus Abb. 2.7. Links ist der Beitrag der inelastischen Prozesse $\delta_{\text {inelast }}$ und rechts die sich ergebende Gesamt-Strahlungskorrektur gezeigt. Die Beiträge der elastischen Prozesse höherer Ordnung werden vom Cut-OffParameter nicht beeinflußt und stimmen mit denen aus Abb. 2.7 überein.

Der Cut-Off-Parameter für die Berechnung der inelastischen Beiträge zu den Strahlungskorrekturen kann nur über eine Simulation des Detektorverhaltens mit entsprechend generierten Ereignissen exakt bestimmt werden. Da das in SELEX verwendete Simulationpaket GEANT über keinen entsprechenden Generator verfügt, kann dieser Parameter nur abgeschätzt werden. Berücksichtigt man, dass der Impuls des Photons in der Impulserhaltung der elastischen Prozesse verloren geht und sich diese mit einer Genauigkeit von rund $5 \%$ bestimmen läßt, so erhält man für einen Strahl 


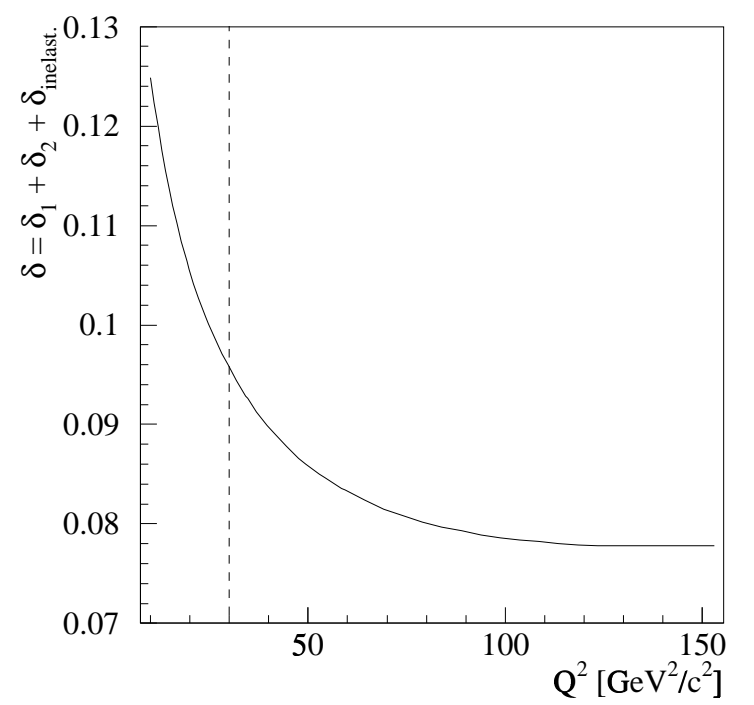

Cut-Off Parameter gegen Strahlungskorrektur

Abbildung 2.9: Abhängigkeit der Gesamt-Strahlungskorrektur, welche als Differenz der Korrektur bei $\mathrm{Q}^{2}=0.3 \mathrm{GeV}^{2} / c^{2}$ und bei $\mathrm{Q}^{2}=0.01 \mathrm{GeV}^{2} / c^{2}$ definiert ist und somit als Maß für die Steigung der Strahlungskorrekturen angesehen werden kann, vom Cut-OffParameter. Eingezeichnet ist der in der Analyse verwendete Cut-Off-Parameter von $30 \mathrm{GeV} / c$.

von $600 \mathrm{GeV} / c$ einen geschätzten Cut-Off-Parameter von rund $30 \mathrm{GeV} / c$. Abbildung 2.8 zeigt zum Vergleich die sich ergebenden inelastischen Korrekturen und die Gesamt-Strahlungskorrekturen für einen Cut-Off-Parameter von 30 und $100 \mathrm{GeV} / c$. Ein Maß für den Einfluß der Strahlungskorrekturen auf den Radius ergibt sich aus dessen Steigung, bzw. aus der Differenz der Korrektur bei $\mathrm{Q}^{2}=0.3 \mathrm{GeV}^{2} / c^{2}$ und bei $\mathrm{Q}^{2}=0.01 \mathrm{GeV}^{2} / c^{2}$. Abbildung 2.9 zeigt die Abhängigkeit dieser Gesamt-Korrektur vom Cut-Off-Parameter. 


\section{Das SELEX Experiment}

In diesem Kapitel soll die Zielsetzung und der Aufbau des Experiments E781 (SELEX) im Experimentierbereich des Proton Center (PC4) am Fermilab ${ }^{4}$ beschrieben werden. Nach einer kurzen Diskussion der physikalischen Schwerpunkte des SELEX Experiments wird das Spektrometer in seinen einzelnen Stufen vorgestellt, bevor die einzelnen Detektoren zur Teilchenidentifikation betrachtet werden. Den Abschluß dieses Kapitels bildet die Beschreibung des Triggers.

\subsection{Allgemeine Ziele des SELEX Experiments}

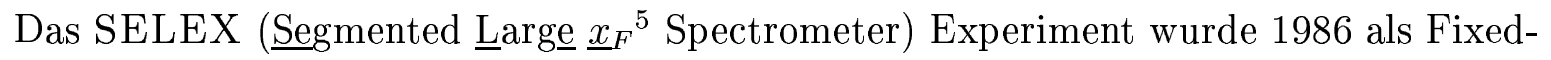
Target der dritten Generation zur Untersuchung von charm-Baryonen vorgeschlagen, nachdem sich gezeigt hatte, dass im Fixed-Target-Experiment WA62 am CERN mit einem Hyperonenstrahl eine weitaus größere Zahl an charm-Baryonen produziert worden war, als in irgendeinem Collider-Experiment zuvor [13]. Letztere produzieren zwar eine Vielzahl an charm-Mesonen, aber nur einen um Faktor zehn kleineren Datensatz an charm-Baryonen. Ein weiterer Vorteil der Fixed-Target-Experimente gegenüber den Collider-Experimenten sind die größeren zur Verfügung stehenden Zerfallslängen für in Vorwärtsrichtung produzierter Teilchen, welche genauere Studien der Lebensdauer ermöglichen.

SELEX kann als direkter Nachfolger des 1994 abgeschlossenen WA89 Experiments am CERN gesehen werden, da beides Fixed-Target-Experimente mit einem Hyperonenstrahl und mit einem ähnlichen Aufbau sind. So verfügen beide über SiliziumMikrostreifen-Detektoren zur präzisen Vertex ${ }^{6}$-Rekonstruktion. Das SELEX Experiment verfügt zusätzlich über eine höhere Strahlintensität, einen längeren duty cycle ${ }^{7}$ und über ein Spektrometer mit höherer Akzeptanz. Weiterhin ist SELEX im Gegensatz zu WA89 mit einem online-charm-Filter ausgestattet, der in einer vorläufige Rekonstruktion den Impact Parameter ${ }^{8}$ berechnet und so eine Reduktion der Rohdaten um einen Faktor vier bis acht ermöglicht.

\subsubsection{Eigenschaften von charm-Baryonen}

Die aus den vier leichtesten Quarks aufgebauten Baryonen lassen sich gruppentheoretisch in zwei 20-pletts mit unterschiedlichem Gesamtspin $J^{P}=\frac{1}{2}^{+}$und $J^{P}=\frac{3}{2}^{+}$ einordnen. Abbildung 3.1 zeigt eine 3-dimensionale Darstellung des symmetrischen und des gemischten Multipletts der SU(4)-Gruppe. Aufgrund der unterschiedlichen Quarkmassen ist die Symmetrie jedoch stark gebrochen. Nicht alle Baryonen dieser Multipletts, vor allem die mit mehreren charm-Quarks, konnten bereits nachgewiesen werden. Eine Übersicht der Eigenschaften einiger charm-Baryonen zeigt Tab. 3.1.

\footnotetext{
${ }^{4}$ eigentlich FNAL - Fermi National Accelerator Laboratory

${ }^{5}$ Die Skalenvariable $x_{F}$ ist über $x_{F}=\frac{\mathrm{Q}^{2}}{2 M \nu}$ mit dem Energietransfer $\nu=E_{\pi}-E_{\pi}^{\prime}$ definiert

${ }^{6}$ engl. Wechselwirkungspunkt

${ }^{7}$ Mit duty cycle bezeichnet man das Produkt aus der Länge des Zeitintervalls, in dem der Teilchenstrahl zur Verfügung steht (dem spill), und der Wiederholungsfrequenz des Intervalls

${ }^{8}$ Der räumliche Abstand zwischen einer Spur im Strahl- und im Vertex-Spektrometer wird als Impact Parameter bezeichnet und dient ab einer Größe von $20 \mu \mathrm{m}$ als Merkmal für eine Wechselwirkung
} 

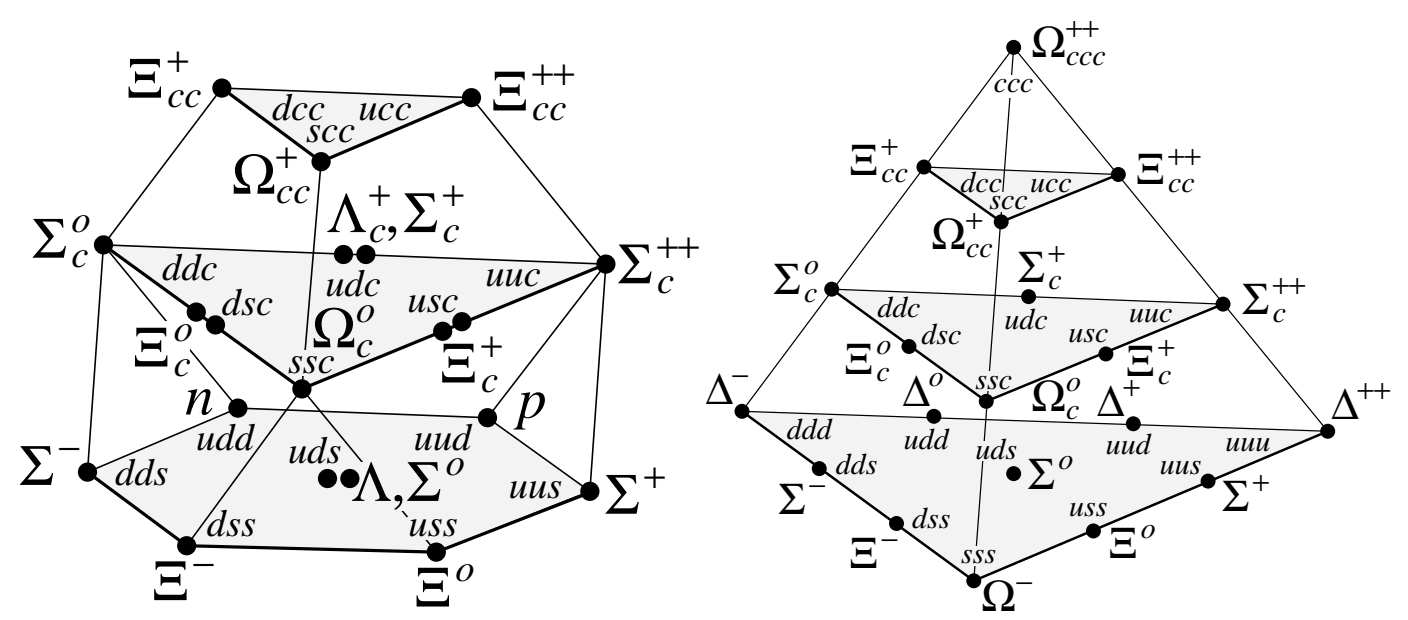

Abbildung 3.1: Die SU(4)-Multipletts der Baryonen aus den vier leichtesten Quarks. Das Baryonen Oktett und Singulett der SU(3)Gruppe bilden die unterste Ebene des 20-pletts der Baryonen mit $J^{P}=\frac{1}{2}^{+}$(links), während das Dekuplett der SU(3)Gruppe die unterste Ebene des SU(4)-Multipletts der Baryonen mit $J^{P}=\frac{3}{2}^{+}$bildet (rechts). Nach rechts ist die Isospinkomponente $I_{3}$, nach hinten die Strangeness und nach oben die Zahl der charm-Quarks aufgetragen.

\subsubsection{Physikalische Schwerpunkte bei SELEX}

Eine Vielzahl verschiedener physikalischer Fragestellungen werden im Rahmen von SELEX untersucht. Als Schwerpunkte sind zu nennen:

Nachweis und Bestimmung der Eigenschaften von charm-Baryonen: Die Eigenschaften vieler charm-Baryonen sind bis heute nur ungenau bestimmt. So sind bis heute die meisten $J^{P}=\frac{3}{2}^{+}$Baryonen mit einem charm-Quark $\left(\Sigma_{c}^{0}, \Sigma_{c}^{+}, \Sigma_{c}^{++}, \Xi_{c}^{0}\right.$ und $\Xi_{c}^{+}$) nicht oder nur in sehr geringer Zahl beobachtet worden. Wie Tab. 3.1 zeigt sind auch zu den charm-Baryonen mit $J^{P}=\frac{1}{2}$ noch manche Eigenschaften unbekannt, insbesonders über die Zerfallskanäle und deren Verzweigungsverhältnisse (bzw. deren partiellen Breiten). Weiterhin sucht SELEX auch nach Baryonen mit 2 charm-Quarks (double charm) [15], welche sich bislang der experimentellen Beobachtung entzogen haben, da die entsprechenden Produktionsquerschnitte um etwa drei Größenordnungen kleiner sind als die der Baryonen mit einem charm-Quark.

Produktionsmechanismen von charm-Baryonen: Die Verwendung verschiedener Strahlteilchen, $\pi^{ \pm}, p$ und $\Sigma^{-}$im Fall von SELEX, erlaubt eine Untersuchung der Produktionsmechanismen von charm-Baryonen. Neben dem leading particle effect, der sich in der bevorzugten Produktion von Teilchen mit möglichst vielen gemeinsamen Konstituentenquarks zeigt, wird allgemein angenommen, dass Baryonen mit schweren Quarks bevorzugt produziert werden, wenn bereits andere schwere Quarks in Strahlteilchen enthalten sind. Da SELEX über verschiedene Targets verfügt und eine große Abdeckung von $x_{F}$ hat, lassen sich sowohl die Abhängigkeiten der Produktionswahrscheinlichkeiten verschiedener Baryonen von $x_{F}$ als auch vom Targetmaterial untersuchen. 


\begin{tabular}{|c|c|c|c|c|c|}
\hline Baryon & $m\left[\mathrm{MeV} / c^{2}\right]$ & $\tau\left[10^{-12} s\right]$ & $c \tau[\mu m]$ & \multicolumn{2}{|c|}{ Zerfallskanäle } \\
\hline$\Lambda_{c}^{+} \quad(\mathrm{udc})$ & $2284.9 \pm 0.6$ & $0.206 \pm 0.012$ & 61.8 & $\begin{array}{l}\Lambda^{0}+X \\
n+X\left(k \text { ein } \Lambda^{0}\right) \\
p+X\left(k \text { ein } \Lambda^{0}\right) \\
\Sigma^{ \pm}+X\end{array}$ & $\begin{array}{l}35 \pm 11) \% \\
(29 \pm 17) \% \\
(12 \pm 19) \% \\
(10 \pm 5) \%\end{array}$ \\
\hline $\begin{array}{l}\Sigma_{c}^{++} \quad \text { (uuc) } \\
\end{array}$ & $2452.9 \pm 0.6$ & - & - & $\overline{\Lambda_{c}^{+}+\pi^{+}}$ & $100 \%$ \\
\hline $\begin{array}{ll}\Sigma_{c}^{+} & (\mathrm{udc})\end{array}$ & $242453.5 \pm 0.9$ & 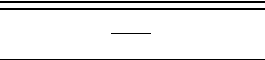 & $\overline{-5}$ & 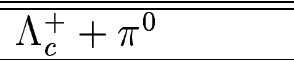 & $\overline{100 \%}$ \\
\hline $\begin{array}{ll}\Sigma_{c}^{0} & (\mathrm{ddc})\end{array}$ & 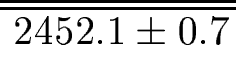 & - & - & $\bar{\Lambda}_{c}^{+}+\pi^{-}$ & $\overline{100 \%}$ \\
\hline $\begin{array}{ll}\Xi_{c}^{+} & \text {(usc) }\end{array}$ & $2465.6 \pm 1.4$ & $0.35_{-0.04}^{+0.07}$ & 106 & $\begin{array}{l}\Lambda^{0} K^{-} \pi^{+} \pi^{+} \\
\Sigma^{+} K^{-} \pi^{+} \\
\Xi^{0} \pi^{+} \\
\Xi^{0} \pi^{+} \pi^{+} \pi^{-} \\
\Xi^{0} e^{+} \nu_{e} \\
\end{array}$ & $\begin{array}{l}\text { beob. } \\
\text { beob. } \\
\text { beob. } \\
\text { beob. } \\
\text { beob. }\end{array}$ \\
\hline $\begin{array}{ll}\Xi_{c}^{0} & (\mathrm{dsc})\end{array}$ & $\overline{2} 2470.3 \pm 1.8$ & $0.098_{-0.015}^{+0.023}$ & 29 & $\begin{array}{l}\Xi^{-} \pi^{+} \\
\Xi^{-} \pi^{+} \pi^{+} \pi^{-} \\
p K^{-} \bar{K}^{*}(892)^{0} \\
\Lambda^{0} \bar{K}^{0} \\
\Omega^{-} K^{+}\end{array}$ & $\begin{array}{l}\text { beob. } \\
\text { beob. } \\
\text { beob. } \\
\text { beob. } \\
\text { beob. }\end{array}$ \\
\hline $\begin{array}{ll}\Omega_{c}^{0} & (\mathrm{ssc})\end{array}$ & $\overline{2704 \pm 4}$ & 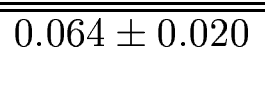 & 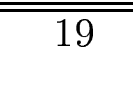 & $\begin{array}{l}\Xi^{-} K^{-} \pi^{+} \pi^{+} \\
\Omega^{-} \pi^{+} \pi^{+} \pi^{-}\end{array}$ & $\begin{array}{l}\text { beob. } \\
\text { beob. }\end{array}$ \\
\hline
\end{tabular}

Tabelle 3.1: Eigenschaften von charm-Baryonen [14].

Schwache Zerfälle: Während die Eigenschaften des $\Lambda_{c}^{+}$relativ gut bekannt sind, gilt den Lebensdauern der charm-strange-Baryonen $\Xi_{c}^{+}, \Xi_{c}^{0}$ und $\Omega_{c}^{0}$ in SELEX ein besonderes Augenmerk. Zum genauen Verständnis der schwachen Zerfälle ist es nötig, den Einfluß der starken Wechselwirkung weit möglichst zu reduzieren [16].

Exotika: Alle bekannte hadronische Materie ist entweder aus zwei (Mesonen) oder drei Quarks (Baryonen) aufgebaut. Da allerdings Mehr-Quark-Zustände nicht prinzipiell verboten sind, versucht man diese in Experimenten mit hoher Statistik nachzuweisen. So gibt es aus der Beobachtung ungewöhnlicher Quantenzahl-Kombinationen Hinweise auf die Existenz sogenannter Dimesonen $(q q \bar{q} \bar{q})[17]$, Pentaquarks (z.B. $P(s \bar{c} q q q)$, $[18,19])$ und Dibaryonen (z.B. das H-Dibaryon mit (udsuds) [20]).

Primakoffproduktion: Die Wechselwirkung geladener Teilchen mit dem Coulombfeld der Kerne führt über den Primakoffeffekt [21], einem Ein-Photonen-Austausch, zur Produktion neutraler Mesonen. Der Primakoffeffekt erlaubt auch das Studium von Polarisierbarkeiten und von Strahlungsübergängen, sowie den Test Chiraler Anomalien.

Totale Wirkungsquerschnitte: Nach der Regge-Theorie ergibt sich ein einfacher Zusammenhang zwischen dem totalen Wirkungsquerschnitt $\sigma_{\text {tot }}$ für die Hadron-ProtonWechselwirkung und der Schwerpunktenergie $s$ zu

$$
\sigma_{t o t}=X s^{\epsilon}+Y s^{-\eta} \text {. }
$$


Hierbei werden $\epsilon$ und $\eta$ als universale Größen angenommen. Diese Annahme läßt sich mit den in SELEX zur Verfügung stehenden Energien testen. Da allerdings in SELEX kein Flüssigwasserstoff-Target verwendet werden kann, wird der Wirkungsquerschnitt über die Differenz der Wirkungsquerschnitte für reinen Kohlenstoff und Polyethylen $\left(\left(\mathrm{CH}_{2}\right)_{n}\right)$ ermittelt $[22]$.

Ladungsradien: Über die elastische Streuung von Hyperonen an den Elektronen eines Targets kann man die elektromagnetischen Formfaktoren von Hyperonen bestimmen [23]. SELEX bietet die Möglichkeit, im Rahmen eines Experiments, Formfaktoren für unterschiedliche Teilchen $\left(\pi^{-}, \Sigma^{-}\right.$und $\left.p\right)$ zu messen [24].

\subsection{Das Spektrometer}

Das $60 \mathrm{~m}$ lange SELEX-Spektrometer ist durch drei Magnete in Bereiche mit unterschiedlicher Impulsabdeckung gegliedert, wobei die erste Stufe Teilchen ab $2.5 \mathrm{GeV} / c$ abdeckt und damit besonders für Teilchen mit geringem Impuls ausgelegt ist, wie z.B. Pionen, die aus dem Zerfall angeregter Zustände stammen. Die zweite Stufe des Spektrometers registriert Teilchen ab $25 \mathrm{GeV} / c$ und ist damit vornehmlich auf die Zerfallsprodukte von charm-Baryonen ausgelegt. In diesem Teil befinden sich auch die Detektoren zur Teilchenidentifikation (siehe 3.3). Dieser zweite Teil des Spektrometers enthält eine $40 \mathrm{~m}$ lange $\Lambda^{0}$-Zerfallszone nach der die $\Lambda^{0}$ 's mit speziell hierfür entsprechenden Driftkammern rekonstruiert werden können. Aufgabe der dritten Spekrometerstufe ist es, die Impulse der Protonen aus den $\Lambda^{0}$-Zerfällen zu bestimmen und Spuren mit hohem Impuls zu vermessen. Abschließend steht ein Neutronenkalorimeter zum Nachweis von Neutronen, z.B. aus dem $\Sigma^{-}$-Zerfall, zur Verfügung. Eine Übersicht über den Aufbau des SELEX-Spektrometers bietet Abb. 3.2.

\subsubsection{Das Strahlspektrometer und der Hyperonen-Strahl}

Das SELEX Experiment wird von einem Strahl gespeist, der zu gleichen Teilen aus $\Sigma^{-}$und $\pi^{-}$besteht, mit einem Impuls von $650 \mathrm{GeV} / c$ und einer Impulsverteilung der Breite von rund 8\%. Dieser Strahl wird rund $11 \mathrm{~m}$ vor dem eigentlichen Spektrometer mit einem Beryllium-Target einer Länge von 0.98 Wechselwirkungslängen aus dem $800 \mathrm{GeV} / c$ Protonenstrahl des Tevatron produziert. Ein Zyklus dauert dabei eine Minute mit 20 Sekunden Spilldauer. Der Teilchenfluß auf das Beryllium-Target beträgt etwa $2 \cdot 10^{12}$ Protonen pro Spill. Aus dem produzierten Teilchenspektrum von $\Sigma^{-}, \bar{p}, \pi^{-}, K^{-}, \Xi^{-}$und $\Omega^{-}$wird mit den Hyperon-Magneten auf das $\Sigma^{-}$als erwünschtes Strahlteilchen selektiert. Hierzu ist in dem Feld der Hyperon-Magneten aus Wolfram und Blei ein Kanal geformt (siehe Abb. 3.3), durch den nur jene Teilchen ihren Weg finden, die das richtige Impuls/Ladungsverhältnis haben. Hierbei wird ein Fluss von ca. $1 \cdot 10^{11} \Sigma^{-}$und $\pi^{-}$produziert. Das Magnetfeld des Hyperon-Magneten lässt sich umpolen, so dass dieser auch zur Selektion von Protonen, $\Sigma^{+}$oder $\pi^{+}$benutzt werden kann. Die Krümmung des Kanals

Um eine genaue Strahldefinition zu erhalten, sind im Strahlbereich Detektoren zur Teilchenidentifikation, zur Ereignisauswahl (Trigger) und zur Spurrekonstruktion untergebracht. 
焉

量

珲

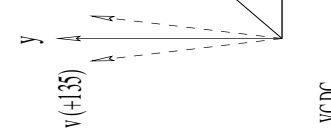

产-
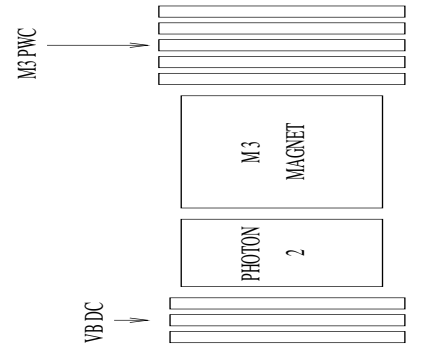

蓄-<smiles>CCCC</smiles>

空

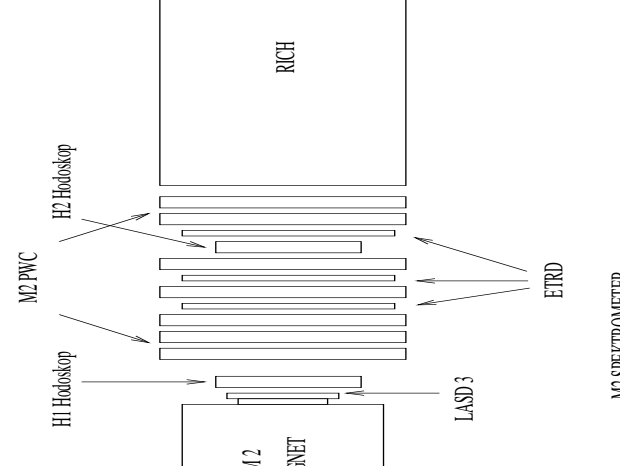



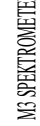

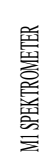<smiles>C1#CCCC#CC1</smiles>
三 萲

甶

旁

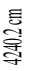

్ㅗำ

罯 통

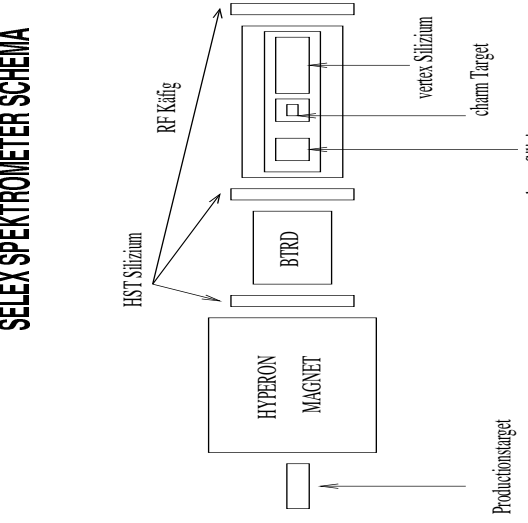
틍 


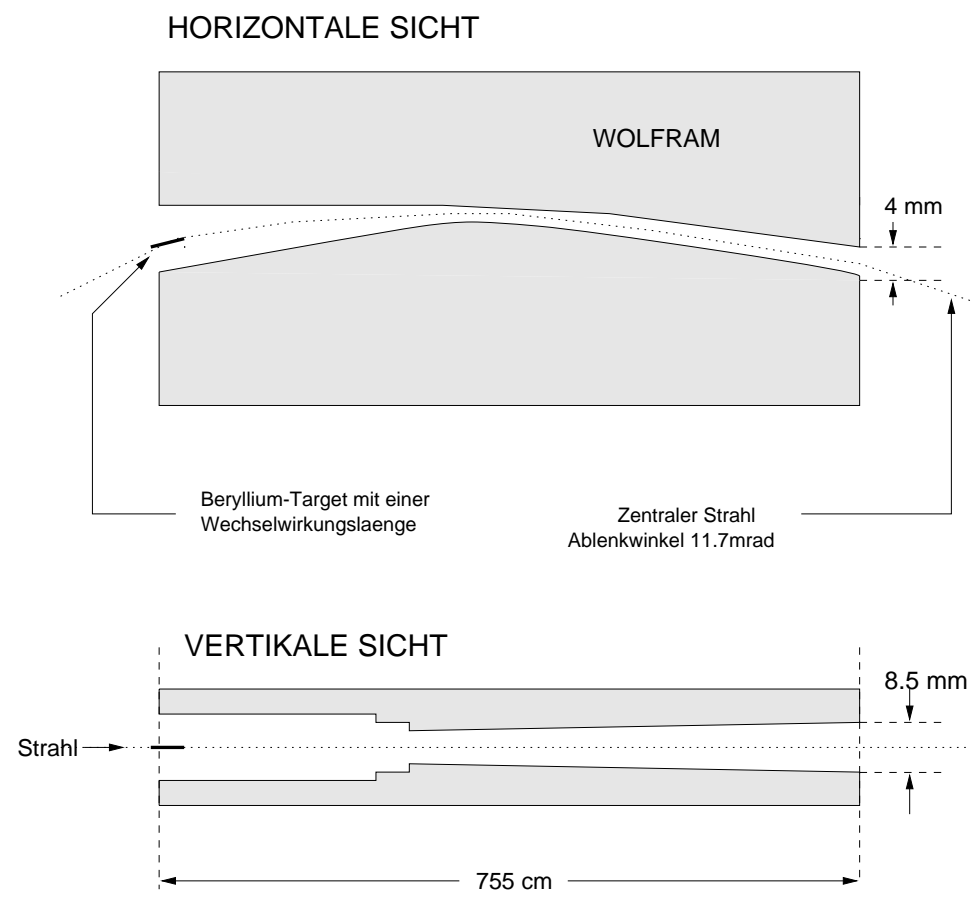

Abbildung 3.3: Layout des Hyperonen-Kanal des SELEX Experiments.

Zur Teilchenidentifikation wird ein Übergangsstrahlungszähler $\left(\right.$ BTRD $\left.^{9}\right)$ verwendet, um Pionen von schweren Strahlteilchen zu unterscheiden. In Abschnitt 3.3 wird auf den BTRD näher eingegangen.

Die erste Stufe der Ereignis-Auswahl erfolgt im Strahlbereich über Plastikszintillatoren (S1-S4 in Abb. 3.4), die den Durchgang eines Teilchens detektieren und deren Signal mit dem Spillsignal des Tevatron verknüpft ist. Über diese Szintillatoren wird die Auslese der drei Silizium-Mikrostreifen-Detektoren $\left(\mathrm{HSD}^{10}\right)$, die Teil des hardware scatter trigger (HST) sind, gesteuert. Je einer der Detektoren steht vor und hinter dem BTRD, sowie der Dritte hinter den Silizium-Mikrostreifen-Detektoren der VertexRegion. Jeder dieser Detektoren besteht aus zwei Ebenen, so dass sich die $x$ - und $y$-Position der durchlaufenden Strahlteilchen bestimmen lassen. Durch eine schnelle Auswertung der Spurinformation der ersten beiden Detektoren und einem anschließenden Vergleich mit den Spuren in dem letzten Detektor lassen sich Streuprozesse identifizieren.

Während die HSD-Module als Teil des HST der Ereignis-Auswahl dienen, wird die Spur des einlaufenden Strahlteilchens mit einem 1m langen Teleskop aus acht $2 \times$

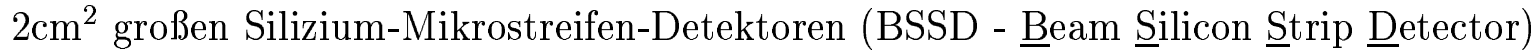
mit einseitiger Auslese bestimmt. Jede Detektorebene besteht aus 1024 Streifen mit

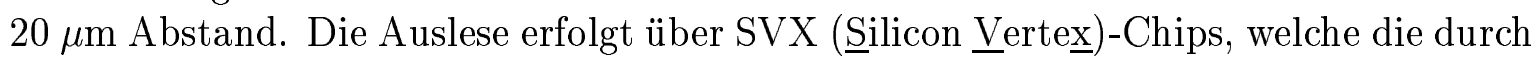
Teilchen freigesetzte Ladung integrieren und diese für eine nullunterdrückte Auslese mit einem Schwellenwert vergleichen. Die große Zahl der Kanäle und die unregelmässige

\footnotetext{
${ }^{9} \underline{\text { Beam }}$ Transition $\underline{\text { Radiation }}$ Detektor

${ }^{10} \mathrm{HST}$ Silicium Detectors
} 


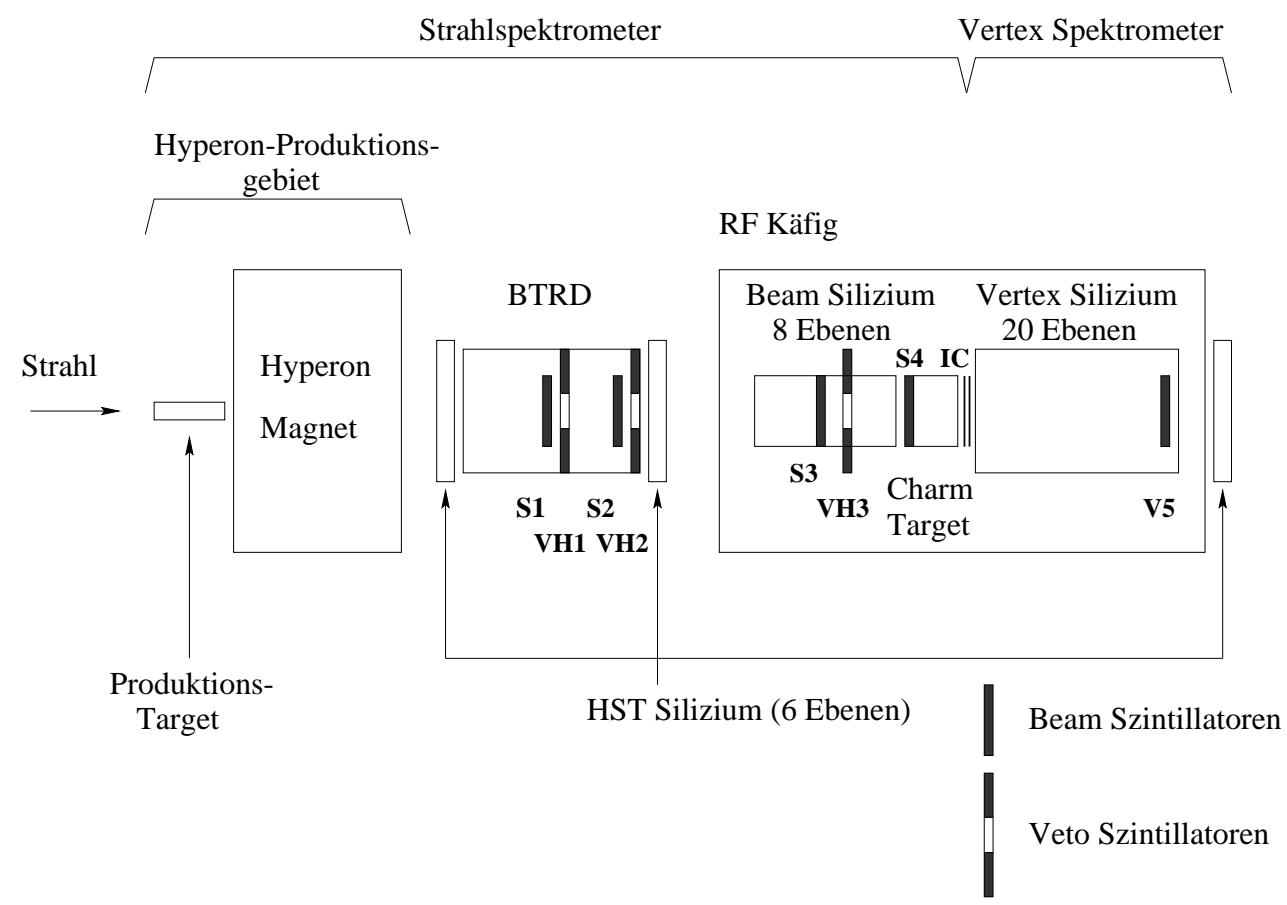

Abbildung 3.4: Aufbau des Strahl- und des Vertex-Spektrometers. Von links das Hyperon-Produktionstarget und der Hyperon-Magnet, der BTRD (Übergangsstrahlungszähler) mit den Szintillatoren zur Strahl-Definition (S1-S4) und zur Strahl-HaloDefinition (VH1-VH3), sowie den HSD-Ebenen.

Zeitstruktur von Fixed-Target-Experimenten erlaubt es nicht, die Silizium-Detektoren nach jedem Strahlteilchen zurück zu setzen. Deswegen werden die Spuren von oftmals mehreren Strahlteilchen, die keine Wechselwirkung im Target erfahren haben ${ }^{11}$, mit ausgelesen, wenn der HST eine Wechselwirkung registiert. Eine typische Verteilung der pro Ereignis rekonstruierten Strahlteilchen zeigt Abb. 3.5. Die exakte Bestimmung der Strahlteilchenbahn erlaubt eine Messung des Strahlimpulses mit einer Auflösung von etwa $0.8 \%$. Die Effizienz der Silizium-Mikrostreifen-Detektoren beträgt ungefähr 98\% bei einer Rekonstruktionswahrscheinlichkeit von 95\%. Die HSD-Module befinden sich im RF-Käfig, indem auch die Targets und das Vertex-Spektrometer untergebracht sind.

\subsubsection{Die Targets und das Vertex-Spektrometer}

SELEX verwendet ein segmentiertes Target aus zwei Kupferfolien von $1.6 \mathrm{~mm}$ und $1.0 \mathrm{~mm}$ Dicke und aus drei Kohlenstofftargets (Industriediamant) von je $2.2 \mathrm{~mm}$ Dicke. Die Dicke der Targets wird durch die Triggerbedingungen für charm-Ereignisse begrenzt, der auf sekundären Zefallsvertizes als Kennzeichen für charm-Zerfälle beruht (s. Abschn. 3.4). Insgesamt haben die Folien für Protonen eine Wechselwirkungslänge von $4.2 \%$. Die Verwendung unterschiedlichen Targetmaterials erlaubt die Untersuchung der Produktionswirkungsquerschnitte für charm-Baryonen in Abhängigkeit zur

\footnotetext{
${ }^{11}$ sogenannte stale tracks
} 

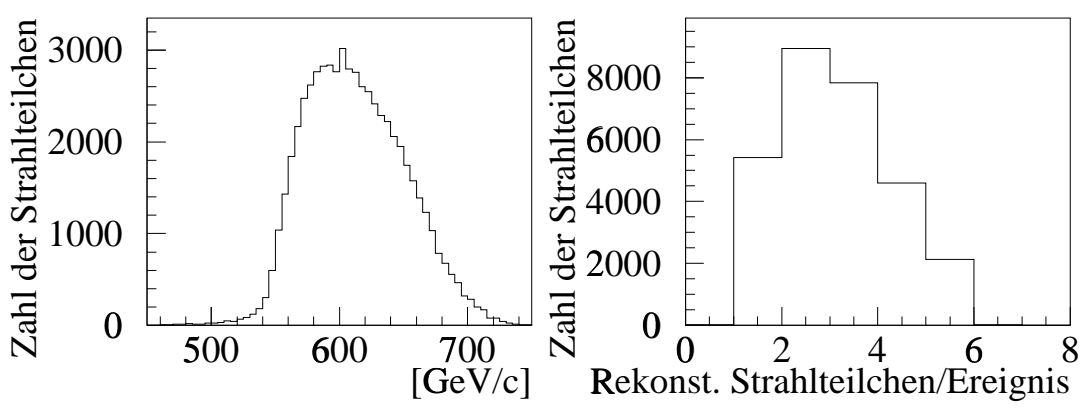

Abbildung 3.5: Strahlimpulsverteilung des SELEX Hyperonenstrahl (links) und die Zahl der rekonstruierten Strahlteilchen pro Ereignis (rechts).

Massenzahl des Targetmaterials. Für die Untersuchung totaler Wirkungsquerschnitte stehen andere Targetkonfigurationen zur Verfügung [22].

Direkt hinter den Targets befindet sich ein Wechselwirkungszähler aus zwei dünnen Plastikszintillatoren. Da die Energieabgabe relativistischer Teilchen in einem dünnen Material für alle Teilchen im Mittel gleich ist, erlaubt die Messung der deponierten Energie eine Bestimmung der Teilchenmultiplizität (s. Abschn. 3.4.1).

Während die acht HSD-Ebenen die einlaufenden Teilchenspuren vermessen, werden die Spuren der auslaufenden Teilchen mit insgesamt 20 Ebenen von SiliziumMikrostreifen-Detektoren (VSSD ${ }^{12}$ ) im Vertexbereich vermessen. Von diesen messen sechs die $x$-Koordinate, vier die $y$-Koordinate und je fünf die $u$ - und $v$-Koordinate. Die Ebenen haben eine Dicke von $300 \mu \mathrm{m}$. Die ersten acht strahlaufwärts gelegenen Detektoren haben einen Streifenabstand von $20 \mu \mathrm{m}$ und eine Fläche von $5 \times 5 \mathrm{~cm}^{2}$, die anderen zwölf Mosaik ${ }^{13}$-Detektoren haben einen Streifenabstand von $25 \mu \mathrm{m}$ bei einer Fläche von $9.6 \times 8.3 \mathrm{~cm}^{2}$. Die VSSD decken einen Winkel von $150 \mathrm{mrad}$ in Vorwärtsrichtung ab und erlauben für Teilchen mit einem Impuls von $100 \mathrm{GeV} / c$ eine Winkelauflösung von $30 \mu \mathrm{rad}$ und eine transversale Ortsauflösung von $4 \mu \mathrm{m}$.

\subsubsection{Das M1-Spektrometer}

Das M1-Spektrometer liegt zwischen den Magneten M1 und M2 und ist für die Rekonstruktion langsamer Teilchen ausgelegt. Der M1-Magnet wird im Standardbetrieb mit einem Magnetfeld von $1.347 \mathrm{~T}$ betrieben, was einem $p_{t}$-kick ${ }^{14}$ von $0.74 \mathrm{GeV} / c$ entspricht. Hierdurch werden geladene Teilchen mit einem Impuls von weniger als $2.5 \mathrm{GeV} / c$ aus der Akzeptanz des M1-Spektrometers herausgelenkt.

Zur Spurrekonstruktion dienen drei Vieldrahtproportionalkammern $\left(\mathrm{MWPC}^{15}\right)$ und zwei Driftkammern $\left(\mathrm{DC}^{16}\right)[25]$.

Drahtkammern bestehen typischerweise aus in einer Ebene gespannten Anodendrähten, die zentral zwischen zwei Kathodenplatten verläuft. Das Füllgas wird von

\footnotetext{
${ }^{12}$ von Vertex $\underline{\text { Silicon }}$ Strip $\underline{\text { Detector }}$

${ }^{13}$ Die Mosaik-Detektoren sind aus drei einzelnen Silizium-Ebenen zusammengesetzt

${ }^{14}$ Die Änderung $\Delta p_{t}$ im transversalen Impuls $p_{t}$ bezeichnet man auch als den $p_{t}$-kick (kick, engl. Stoß)

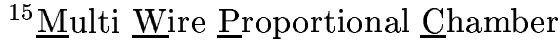

${ }^{16}$ Drift $\underline{\text { Chamber }}$
} 
durchfliegenden Teilchen ionisiert und die Elektronen wandern durch das Potential zum nächstgelegenen Anodendraht. Auf dem Weg dorthin ionisieren die beschleunigten Elektronen weitere Gasmoleküle, was eine Verstärkung des Signals zur Folge hat. Die entstehende Lawine verläuft tropfenförmig, da die Elektronen eine größere Beweglichkeit haben [26].

Jede MWPC-Station umfasst vier Drahtkammern mit je 640 Drähten, welche in 3 mm Abstand zueinander verlaufen und die vier Koordinatenrichtungen $x, y, u$ und $v$ abdecken. Die Fläche der MWPC beträgt $2 \times 2 \mathrm{~m}^{2}$ und als Füllgas wird eine Mixtur von Argon (75\%), Isobutan(24.5\%) und Freon(9.5\%) verwendet. Die Auflösung der MWPCs beträgt ca. $0.6 \mathrm{~mm}$.

Die Driftkammern (DCs) sind ähnlich gebaut wie die MWPC, nur nutzen sie zusätzlich den zeitlichen Abstand des Signals relativ zur Durchgangszeit des Teilchens aus. Der Abstand zwischen den Anodendrähten und der Kathodenplatte wird hierfür von meist 3-8 mm auf 10-50 mm erhöht. Das elektrische Feld muss allerdings mit zusätzlichen Kathodendrähten homogenisiert werden, um so eine einheitliche Driftgeschwindigkeit der Elektronen zu ermöglichen [26].

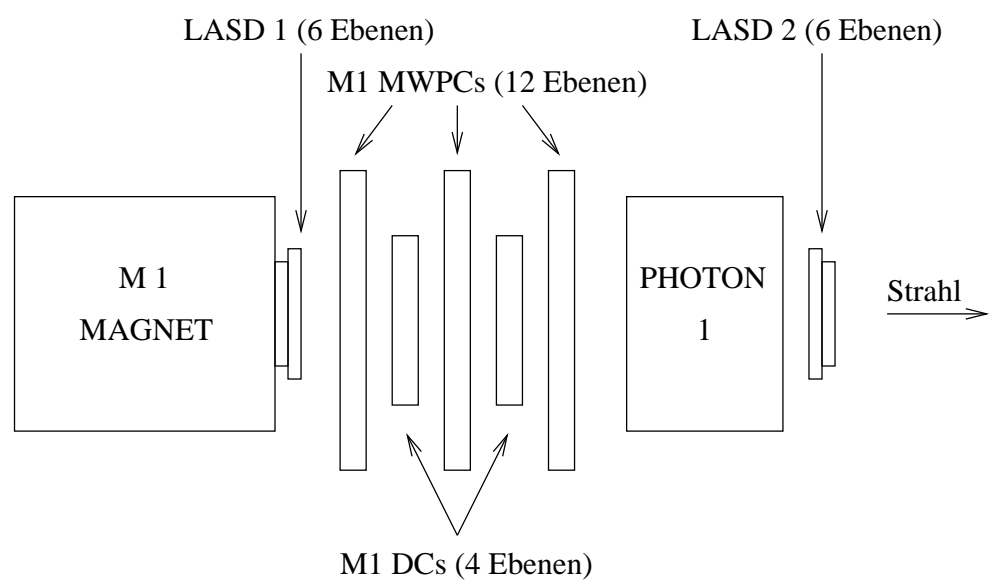

Abbildung 3.6: Layout des M1-Spektrometers.

Wegen der hohen Raten im zentralen, strahlnahen Bereich können die Proportionalkammern hier keine sinnvollen Informationen liefern. Um auch in Strahlnähe eine hohe Spur- und Impulsauflösung zu gewährleisten, werden die Proportionalkammern durch drei weitere Silizium-Mikrostreifen-Detektoren ergänzt. Am Ausgang des M1Magneten, sowie am Ein- und Ausgang des M2-Magneten sind LASDs ${ }^{17}$ [27] angebracht. Jeder der LASD-Stationen besteht aus sechs Ebenen, zwei jeweils aus $63,5 \times$ $63,5 \mathrm{~mm}^{2}$ großen einseitigen ( $\mathrm{SSD}^{18}$ ) und zwei jeweils aus $64,4 \times 51,6 \mathrm{~mm}^{2}$ großen zweiseitigen Detektoren $\left(\mathrm{DSD}^{19}\right)$, beide mit jeweils $50 \mu \mathrm{m}$ Ausleseabstand. Die zweiseitigen Detektoren bieten den großen Vorteil, dass sie, im Vergleich zu den einseitigen Detektoren, nur die Hälfte an Material in den Strahl stellen, was die Wahrscheinlichkeit von unerwünschten, zusätzlichen Wechselwirkungen senkt. SELEX war das erste Experiment, das solche DSDs mit nullunterdrückter Auslese verwendet hat. Mit den LASDs

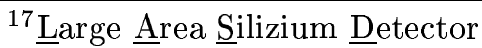

${ }^{18} \underline{\text { Single }}$ Sided Detector

${ }^{19}$ Double $\underline{\text { Sided }}$ Detector
} 
wird eine Impulsaufösung im zentralen Bereich von $\sigma_{p} / p^{2} \approx 1,7 \cdot 10^{-5} \mathrm{GeV}^{-1} c$ erreicht.

Hinter den Proportionalkammern steht der erste Teil eines dreiteiligen elektromagnetischen Kalorimetersystems aus segmentiertem Bleiglas. Das erste Kalorimeter (Photon-1) deckt einen Winkelbereich von 30 bis 100 mrad ab und dient vor allem der Rekonstruktion von $\pi^{0}$ 's aus ihren Zerstrahlungsphotonen. Im M2- und M3-Spektrometer stehen die weiteren Teile, die den verbleibenden Winkelbereich von kleiner $30 \mathrm{mrad}$ in Vorwärtsrichtung abdecken. Insgesamt ist das M1-Spektrometer für die Spektroskopie von angeregten Baryon-Zuständen ausgelegt. Angeregte Baryonen zerfallen in der Regel über weiche Pionen, also Pionen mit geringem Impuls.

\subsubsection{Das M2-Spektrometer}

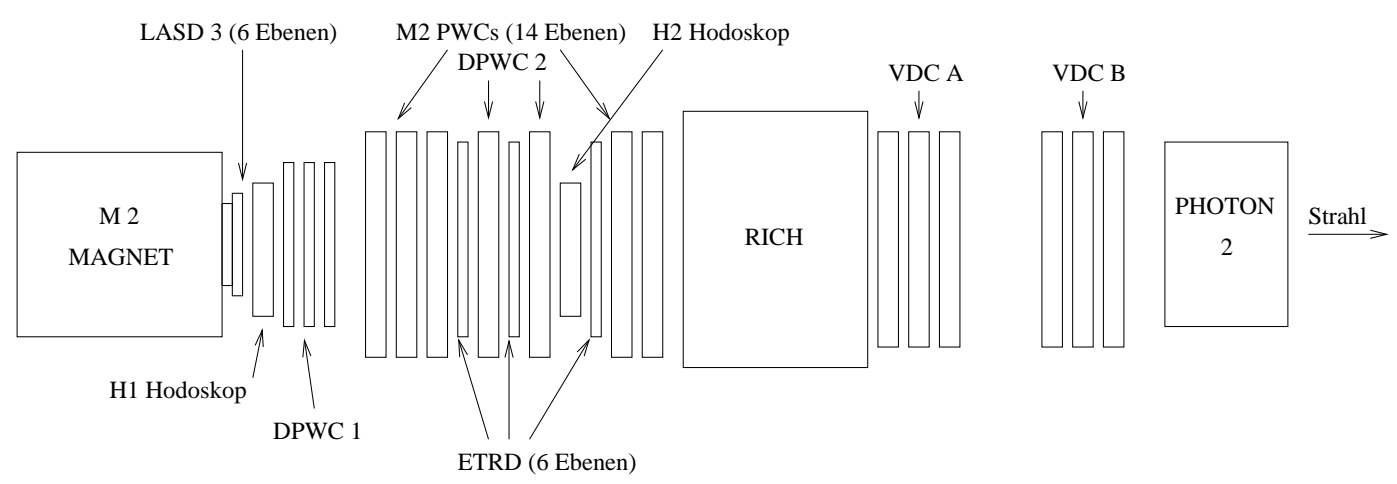

Abbildung 3.7: Layout des M2-Spektrometers.

Mit einem Magnetfeld von $1.541 \mathrm{~T}$ oder einem $p_{t}$-kick von $0.845 \mathrm{GeV} / c$ lenkt der M2-Magnet alle Teilchen mit einem Impuls von weniger als $15 \mathrm{GeV} / c$ aus der Akzeptanz des M2-Spektrometers. Am Ausgang des M2-Magneten ist die letzte LASDStation, gefolgt vom ersten Hodoskop (H1), montiert. Die zwei im M2-Spektrometer integrierten Hodoskope sind beide für den Einsatz im Trigger vorgesehen. Sie liefern mit ihren vertikal verlaufenden Szintillatoren sowohl Multiplizitäts-, als auch, da hinter den Magneten M1 und M2 stehend, Ladungs- und Impulsinformationen (s. auch Abschn. 3.4.2).

Der Spurrekonstruktion dienen sieben MWPCs im vorderen Bereich des M2-Spektrometers. Jeder dieser sieben MWPCs besteht aus zwei Drahtkammern, die entweder die $x$ - und $y$ - oder die $u$ - und $v$-Koordinate abdecken. Die ersten zwei MWPCs überdecken eine Fläche von $60 \times 60 \mathrm{~cm}^{2}$ und die restlichen eine Fläche von $100 \times 60 \mathrm{~cm}^{2}$. Wie die M1-Kammern haben sie einen Drahtabstand von $2 \mathrm{~mm}$. Im hinteren Teil des M2-Spektrometers stehen zwei der drei VEE-Drahtkammern ${ }^{20}\left(\mathrm{VDCs}^{21}\right)$. Diese drei VEE-Stationen (VDC A, B und C) stehen in jeweils fünf, bzw. zehn Metern Abstand zueinander und bestehen aus drei Kammern, die jeweils eine Koordinate messen [28].

Zur Teilchenidentifikation stehen im M2-Spektrometer drei verschiedene Systeme zur Verfügung. Der Elektronenübergangsstrahlungszähler $\left(\mathrm{ETRD}^{22}\right)$, das ringabbil-

\footnotetext{
${ }^{20}$ Der Name rührt von der charakteristischen V-Signatur der $\Lambda^{0}$-Zerfälle in $\pi^{-}$und $p$ her

${ }^{21}$ Vector Drift $\underline{\text { Chamber }}$

${ }^{22}$ Electron Transition $\underline{\text { Radiation }}$ Detector
} 
dende Čerenkov-Teleskop $\left(\mathrm{RICH}^{23}\right)$ und am Ende des M2-Spektrometers die zweite Photon-Kalorimeter-Station. Diese Systeme werden im Abschnitt zur Teilchenidentifikation (Abschn. 3.3) näher betrachtet.

\subsubsection{Das M3-Spektrometer}

Das M3-Spektrometer bildet den Abschluss des SELEX-Spektrometers. In ihm werden vor allem die Zerfallsprodukte von langlebigen Hyperonen, wie z.B. des $\Lambda^{0}$, nachgewiesen. Der M3-Magnet mit einem Feld von $1.3 \mathrm{~T}$ erzeugt einen $p_{t}$-kick von $0.72 \mathrm{GeV} / c$.

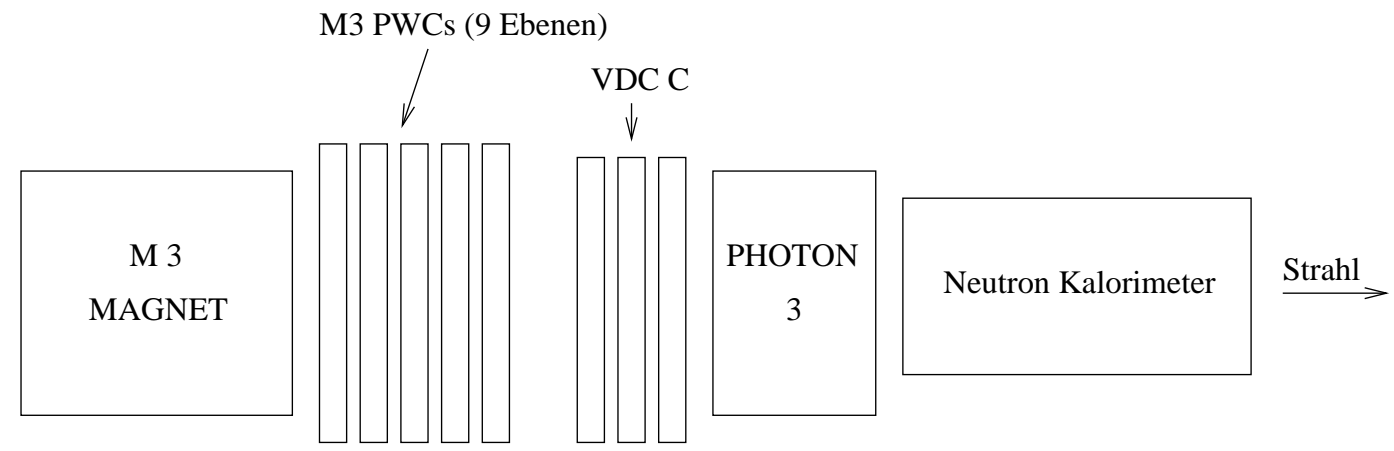

Abbildung 3.8: Layout des M3-Spektrometers.

Auf den M3-Magneten folgen drei MWPC-Stationen, mit einer Fläche von $64 \times$ $64 \mathrm{~cm}^{2}$ für die ersten beiden und $115 \times 89 \mathrm{~cm}^{2}$ für die letzte. Die MWPCs haben, wie die entsprechenden Kammern im M2-Spektrometer, einen Drahtabstand von $2 \mathrm{~mm}$ und decken alle vier Koordinaten $x, y, u$ und $v$ ab. Den MPWCs ist die letzte VEEDrahtkammer (C-VDC) nachgeschaltet.

Das M3-Spektrometer wird durch zwei Kalorimeter abgeschlossen. Zuerst folgt die dritte und letzte Station des elektromagnetischen Kalorimeters (Photon-3; s. Seite 23) und dann das hadronische Kalorimeter $\left(\mathrm{NCAL}^{24}\right)$, zur Messung der Energie von hadronischen Zerfallsprodukten $(n$ und $p$ ) von Hyperonen.

\subsection{Teilchenidentifikation}

Zur Teilchenidentifikation stehen SELEX zwei Übergangsstrahlungszähler (BTRD und ETRD), ein ringabbildendes Čerenkov-Teleskop (RICH), ein dreigeteiltes elektromagnetisches (PHOTON 1-3) und ein hadronisches Kalorimeter (NCAL) zur Verfügung. Der erste Übergangsstrahlungszähler (BTRD) steht im Strahlbereich vor den Targets und der zweite (ETRD) steht im M2-Spektrometer vor dem RICH. Die drei Teile des elektromagnetischen Kalorimeters stehen jeweils am Ende der drei Spektrometer M1 bis M3. Das hadronische Kalorimeter bildet den Abschluss des SELEX-Spektrometers. 




Abbildung 3.9: Der Strahl Übergangsstrahlungszähler BTRD erlaubt die Trennung von $\pi^{-}$und den schweren $\Sigma^{-}$über die Zahl der aktiven BTRD Ebenen. Die Schnitte der Teilchenidentifizierung mit 4, bzw. 7 Ebenen sind markiert.

\subsubsection{BTRD}

Die Strahlung, welche geladene Teilchen beim Übergang zwischen Medien verschiedener Dielektrizität emittieren, ist ungefähr proportional zum relativistischen LorentzFaktor $\gamma=E / m c^{2}$, welcher bei einem typischen Strahlimpuls von $650 \mathrm{GeV} / c$ für $\pi^{-}$ $\gamma_{\pi}=5000$ und für $\Sigma^{-} \gamma_{\Sigma}=550$ beträgt. Die beim Übergang abgestrahlten Photonen haben eine Energie von typischerweise einigen $\mathrm{keV}$ und lassen sich mit Vieldrahtproportionalkammern nachweisen. Deswegen besteht daer BTRD aus 10 Modulen von je 200 Polypropylenfolien und 3 Proportionalkammern. Die Folien haben eine Dicke von $17 \mu \mathrm{m}$ und einen Abstand von $0.5 \mathrm{~mm}$. Die mit einem Gemisch aus Xenon und $30 \%$ Methan gefüllten Proportionalkammern haben einen Driftabstand von $2 \mathrm{~mm}$ und bestehen aus $15 \mu \mathrm{m}$ dicken, goldbeschichteten Wolframdrähten mit $1 \mathrm{~mm}$ Abstand [29].

Abbildung 3.9 zeigt eine typische Verteilung der Module mit positivem Signal. Die Identifikation für $\Sigma^{-}$und $\pi^{-}$erfolgt über die Zahl der aktivierten Ebenen. Bei weniger als 4 Ebenen beträgt die Wahrscheinlichkeit für ein $\Sigma^{-} 91.5 \%$, mit einem Untergrund von 2.2 bis $6 \% \pi^{-}$. Bei mehr als 7 Ebenen mit Aktivität beträgt die Wahrscheinlichkeit für ein $\pi^{-} 89.5 \%$, mit einem Untergrund von 3.3 bis $5.7 \% \Sigma^{-}$.

\subsubsection{ETRD}

Der ETRD besteht aus sechs Modulen aus je einer die $x$-Koordinate messenden Vieldrahtproportionalkammer, dem ein Radiator aus 200 Polypropylenfolien vorgeschaltet ist. Die Folien haben eine Dicke von $17 \mu \mathrm{m}$ und eine Abstand von $0.5 \mathrm{~mm}$. Die Drahtkammern haben einen Drahtabstand von $4 \mathrm{~mm}$ und decken mit ihren 256 Drähten eine Fläche von $103 \times 63 \mathrm{~cm}^{2}$ ab. Das Füllgas ist ein Gemisch aus Xenon und Methan (30\%).

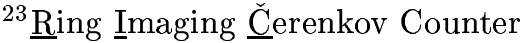

${ }^{24}$ Neutron Calorimeter
} 

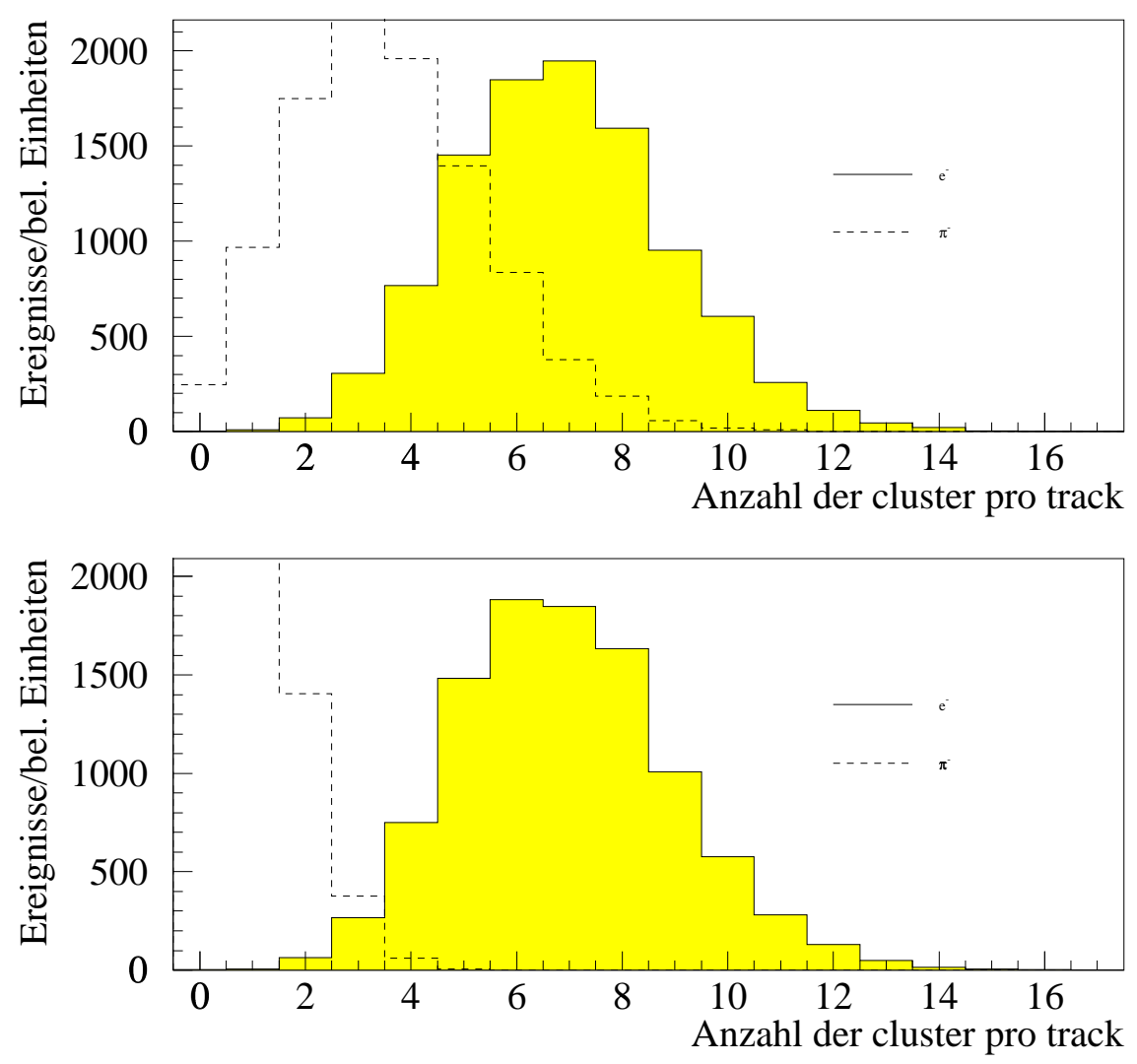

Abbildung 3.10: Verteilung der ETRD Cluster für Elektronen und Pionen von $200 \mathrm{GeV} / c$ (oben) und $20 \mathrm{GeV} / c$ (unten).

Der ETRD arbeitet nach der cluster-counting-Methode, bei der die Anzahl der Ladungscluster ausgegeben wird, die während einer festen Integrationszeit einen eingestellten Schwellenwert überschritten haben. Eine typische Verteilung der Zahl der Cluster für 20 und $200 \mathrm{GeV} / c$ Elektronen und Pionen zeigt Abb. 3.10[30].

\subsubsection{RICH}

Zur Trennung von Pionen, Kaonen und Protonen ist im M2-Spektrometer ein ringabbildender Čerenkovdetektor installiert. In dessen mit Neon gefüllten 10 m langen zylindrischen Tank von $2.4 \mathrm{~m}$ Durchmesser strahlen geladene Teilchen, deren Geschwindigkeit größer ist als die von Licht in Neon, Photonen auf einem Kegel mit einem bestimmten Öffnungswinkel $\theta_{c}$ ab. Dieser Öffnungswinkel

$$
\theta_{c}=\arccos \frac{1}{\beta n(\omega)} \approx \sqrt{2(1-1 / n \beta)}
$$

hängt vom Brechungsindex $n$ des Radiators und der Geschwindigkeit $v=\beta c$ des Teilchens ab, wobei $\omega$ die Frequenz des emittierten Lichtes ist. Ist der Impuls eines einfliegenden Teilchens bekannt, so kann aus dem Öffnungswinkel des Čerenkovlichtes die Masse des Teilchens abgeschätzt werden (Abb. 3.11).

Das Čerenkov licht wird von 16 hexagonförmigen Spiegeln am Tankende auf eine Matrix von 2848 Photomultiplieren reflektiert. Diese mißt einen Kegelschnitt (Kreis), 


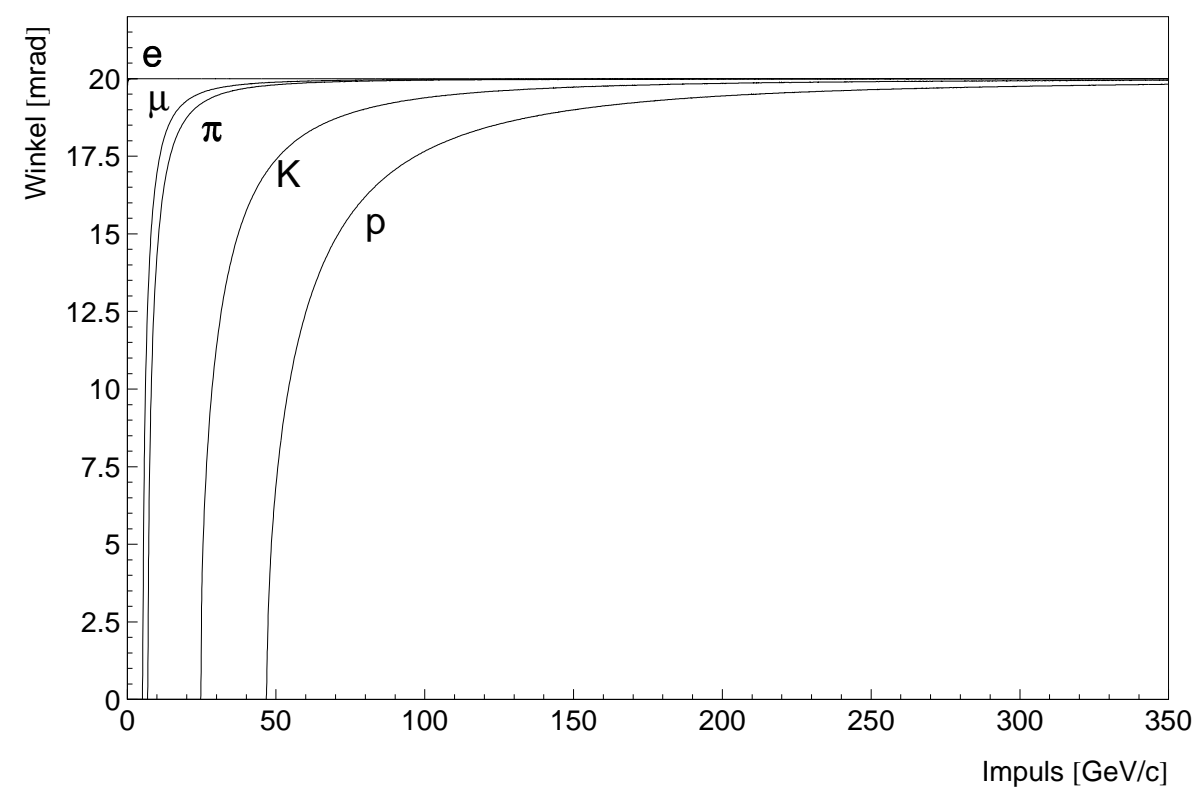

Abbildung 3.11: Impulsabhängigkeit des Čerenkov winkels für verschiedene Teilchen in einem Medium mit Brechungsindex $n=1.0002$.

den sie durch den Čerenkov lichtkegel legt.

\subsubsection{Die Kalorimeter}

Als dritte Detektorart zur Teilchenidentifikation stehen zwei Kalorimeter im SELEX Strahl.

Ein hochenergetisches Photon produziert in Materie einen elektromagnetischen Schauer aus Elektronen und Positronen, welche ihrerseits wieder hochenergetische Bremsstrahlungsphotonen emittieren, solange ihre Energie groß genug ist, so dass die Bremsstrahlung den Energieverlust gegenüber Streuprozessen dominiert. Die hochrelativistischen Elektronen und Positronen strahlen Čerenkov licht ab, welches von Photomultiplieren gesammelt wird.

Das elektromagnetische Kalorimeter deckt mit seinen drei Stationen insgesamt einen Winkel von 100 mrad um den Strahl herum ab. Jede dieser Stationen besteht aus einem Array von Bleiglassteinen mit einem Loch im zentralen Strahlbereich. Die Bleiglassteine haben eine Dichte von $4.1 \mathrm{~g} / \mathrm{cm}^{3}$ und eine Strahlungslänge von $2.5 \mathrm{~cm}$. Die ersten beiden Stationen bestehen aus Steinen zweier verschiedener Größen von jeweils $4.25 \times 4.25 \times 34 \mathrm{~cm}^{3}$, bzw. $8.25 \times 8.25 \times 34 \mathrm{~cm}^{3}$. Die Länge entspricht 13.6 Strahlungslängen. Die Bleiglassteine der dritten Station haben die Maße $3.8 \times 3.85 \times 45 \mathrm{~cm}^{3}$. Die Zahl der Bleiglassteine, die abgedeckte Fläche und die Größe des zentralen Loches sind der Tab. 3.2 zu entnehmen.

Die zusammengesetzte Bauweise aus einzelnen Steinen erlaubt eine Abschätzung der Position des durchgehenden Teilchens. Hierzu bestimmt man die Verteilung der depositionierten Energie, wobei die deponierte Energiemenge über die Integration der Pulshöhen abgeschätzt werden kann [31].

Das NCAL besteht aus 50 Szintillatoren, die in einer Sandwich-Anordnung zwischen 


\begin{tabular}{|l|rr|r|c|c|} 
& \multicolumn{2}{|c|}{ Blöcke } & $z$-Position & \multicolumn{2}{|c|}{ Transversale Ausdehnung } \\
& kleine & große & {$[\mathrm{cm}]$} & total $\left[\mathrm{cm}^{2}\right]$ & Loch $\left[\mathrm{cm}^{2}\right]$ \\
\hline \hline Photon 1 & 576 & 54 & 526.6 & $136.0 \times 110.5$ & $42.5 \times 17.0$ \\
Photon 2 & 540 & 186 & 3573.2 & $229.5 \times 110.5$ & $50.7 \times 41.2$ \\
Photon 3 & 328 & - & 5003.9 & $80.2 \times 61.1$ & $15.3 \times 7.6$ \\
\hline
\end{tabular}

Tabelle 3.2: Spezifikationen der elektromagnetischen Kalorimeter.

50 Stahlplatten eingelassen sind. Zusätzlich stehen im strahlaufwärtsgelegenen Teil noch 17 Drahtkammern zur Verfügung. Insgesamt hat das NCAL eine Länge von 15.6 Strahlungslängen.

\subsection{Der Trigger}

Bei einer Strahlrate von 10 Million Teilchen pro 20 Sekunden Spill liegen zwischen zwei Ereignissen durchschnittlich nur $2 \mathrm{~ms}$. Eine solche Rate von $0.5 \mathrm{MHz}$ stellt hohe Anforderungen an die Datennahme. Die komplette Auslese des SELEX Detektors liefert eine Datenmenge von 5 kilobyte pro Ereignis, was bei 100000 erwarteten, getriggerten Ereignis pro Spill zu einem Datenstrom von 500 megabyte pro Minute führt. Strenge Anforderungen an Ereignisselektion ergeben sich aus der nicht zu vernachlässigenden Totzeit von $30 \mu$ s während der Auslese, die in Einzelfällen bis $50 \mu$ s dauern kann.

Der SELEX-Hardware-Trigger ist in drei Stufen gegliedert, auf die als vierte Stufe ein Software-Trigger aufsetzt [32, 33]. In der zweiten und dritten Stufe des HardwareTriggers kann auf verschiedene Ereignisarten getriggert werden. Hierbei sind $90 \%$ der Bandbreite dem charm-Trigger vorbehalten. Der Hadron-Elektron-Trigger und später andere Trigger laufen parallel zum charm-Trigger.

Im Folgenden werden zuerst die zur Verfügung stehenden Triggerelemente vorgestellt und im Anschluss daran wird die Triggerlogik, also die Zusammenstellung der einzelnen Triggerelemente, diskutiert.

\subsubsection{Der Wechselwirkungszähler}

Der mittlere Energieverlust $d E$ geladener Teilchen pro Wegelement $d x$ (in $\mathrm{g} / \mathrm{cm}^{2}$ ) in Materie durch die Wechselwirkung mit Hüllenelektronen wird durch die Bethe-BlochFormel beschrieben

$$
-\frac{d E}{d x}=K z^{2} \frac{Z}{A} \frac{1}{\beta^{2}}\left[\frac{1}{2} \ln \frac{2 m_{e} c^{2} \beta^{2} \gamma^{2} T_{\max }}{I^{2}}-\beta^{2}-\frac{\delta}{2}\right] .
$$

Eine Diskussion dieser Formel findet sich in jedem Lehrbuch der Teilchenphysik und soll hier nicht näher diskutiert werden (siehe z.B. [14]). Eine Übersicht der verwendeten Konstanten findet sich im Anhang C.

Aus der Bethe-Bloch-Formel ergibt sich für den bei der elastischen Streuung interessanten Energiebereich, für alle Teilchen unabhängig von deren Masse, ein mittlerer Energieübertrag von rund $200 \mathrm{keV} / \mathrm{mm}$, was zur Multiplizitätsmessung über die Energiedeposition genutzt werden kann. Die Genauigkeit dieser Methode ist allerdings begrenzt, da der Energieübertrag Landau-verteilt [34] ist. 


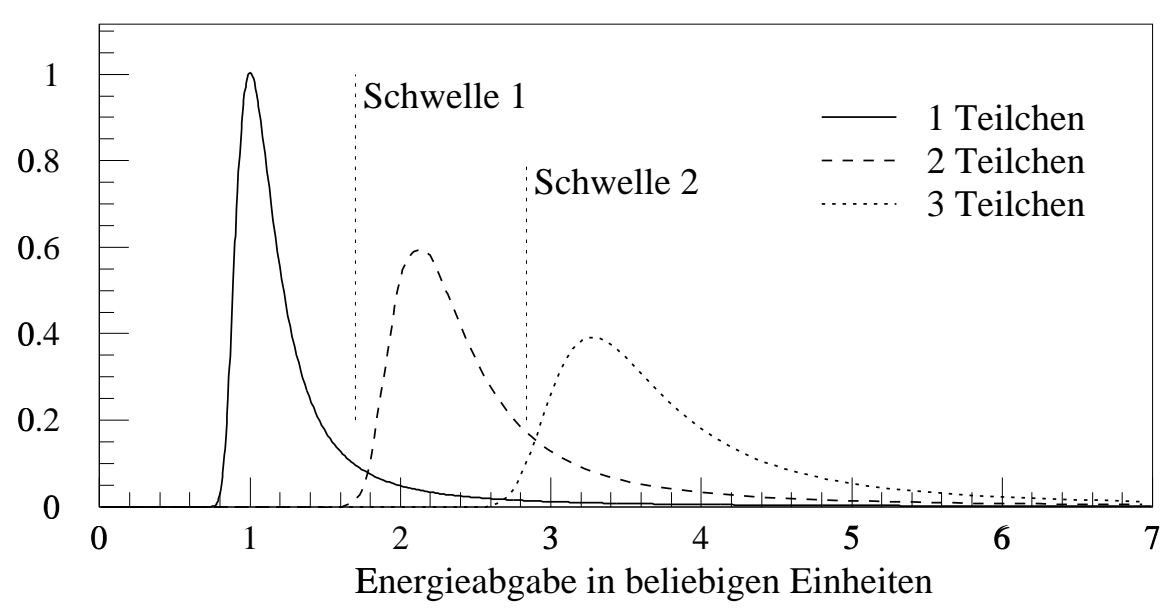

Abbildung 3.12: Idealisiertes Bild zur Multiplizitätstrennung mit einem Szintillator über die Energiedeposition. Möglichen Schwellen zur Multiplizitätstrennung sind eingezeichnet.

Dabei ist nur jener Teil der Energiedeposition interessant, der innerhalb des Wechselwirkungszählers detektiert werden kann. Da das verwendete Szintillatormaterial eine in Strahlungslängen nur geringe Breite hat, entkommen die meisten Photonen der Bremsstrahlung, da diese nicht konvertiert werden. Auch sehr große Energieüberträge bei denen $\delta$-, bzw. Knock-Out-Elektronen entstehen, sind nicht meßbar, da diese aus dem endlichen Detektorvolumen herausfliegen. Die gemessene Energiedeposition stammt vornehmlich aus Stoßionisationen.

Der Wechselwirkungszähler $\left(\mathrm{IC}^{25}\right)$ besteht aus zwei $2 \mathrm{~mm}$ dicken Plastikszintillatoren, die durch Lichtleiter an Photomultiplier gekoppelt sind. Dabei ist das zeitliche Integral des Signals proportional zur Lichtmenge und damit zur Energiedeposition [35].

Besonders wichtig ist die Trennung von Ein- und Zwei-Teilchen-Ereignissen, da die Zahl der Ein-Teilchen-Ereignisse um mehrere Größenordnungen größer ist, als die zu selektierenden elastischen Zwei-Teilchen-Ereignisse. Die Diskriminierung zwischen diesen beiden Ereignisarten erfolgt aufgrund der Landaufluktuationen über den Vergleich der Signale der beiden Szintillatoren, bei der Wahl des kleineren Signals.

Für die Multiplizitätsbestimmung mit dem Wechselwirkungszähler müssen Schwellen für die Trennung der Multiplizität vorgegeben werden. Abbildung 3.12 zeigt das Prinzip dieser Wahl der Schwellen für die Trennung von Ein-, Zwei- und Drei-Teilchenereignissen.

\subsubsection{Die Hodoskope}

Das M2-Spektrometer beinhaltet zwei Hodoskope ${ }^{26}$, die zu Triggerzwecken genutzt werden. Die Hodoskope bestehen aus segmentierten Szintillatoren, die je ein schmales $x$-Segment vertikal abdecken, was eine Ladungstrennung ermöglicht. Das Hodoskop 1 besteht aus 38 Elementen, deren Breite von $5 \mathrm{~mm}$ im zentralen Bereich bis zu $40 \mathrm{~mm}$ für die äußersten Elemente des Hodoskops reicht. Das Hodoskop 2 besteht aus 60

\footnotetext{
${ }^{25}$ Interaction $\underline{\text { Counter }}$

${ }^{26}$ zu gr. hodós "Weg“"und gr. skopeĩn „betrachten, beschauen“
} 


\begin{tabular}{|c|c|c|ccc|}
\hline & z.-pos. & bedeckte Fläche & \multicolumn{3}{|c|}{ Elemente } \\
& {$[\mathrm{cm}]$} & {$\left[\mathrm{cm}^{2}\right]$} & - & 0 & + \\
\hline \hline H1 & 890.9 & $60.0 \times 30.5 \mathrm{~cm}^{2}$ & 16 & 8 & 14 \\
H2 & 1292.1 & $119.0 \times 40.6 \mathrm{~cm}^{2}$ & 12 & 16 & 32 \\
\hline
\end{tabular}

Tabelle 3.3: Spezifikationen der beiden Hodoskope. Beide, H1 und H2, sind gruppiert in die Bereiche negativer Ladungen (-), positiver Ladungen $(+)$ und den zentralen Bereich des M2-Spektrometers.

Elementen, ebenfalls mit Breiten von 5 bis $40 \mathrm{~mm}$. Beide Hodoskope sind in drei logische Bereiche eingeteilt, die den Bereich negativer Ladung (-), positiver Ladung $(+)$, sowie den zentralen Bereich (0) abdecken. Tabelle 3.3 zeigt die Verteilung der Elemente auf diese drei Bereiche und die abgedeckte Fläche, sowie die $z$-Position der Hodoskope.

\subsubsection{Die Triggerlogik}

Im Folgenden werden die einzelnen Stufen der Triggerlogik diskutiert, wobei ab der zweiten Trigger-Stufe T1 nur auf die Eigenschaften des Hadron-Elektron-Triggers eingegangen wird [36].

Triggerstufe T0: Die erste Stufe des Triggers T0 prüft die Strahldefinition über die Szintillatoren S1-S4 und die Veto-Szintillatoren VH1-VH2 (Abb. 3.4, S. 20)

$$
\mathrm{T} 0=\mathrm{S} 1 \cdot \overline{\mathrm{VH} 1} \cdot \mathrm{S} 2 \cdot \overline{\mathrm{VH} 2} \cdot \mathrm{S} 3 \cdot \overline{\mathrm{VH} 3} \cdot \mathrm{S} 4 \cdot \mathrm{V} 5
$$

Die Veto-Szintillatoren haben an der Soll-Position des Strahls ein Loch, so dass sie nur bei Strahlteilchen, die nicht aus dem mittleren Bereich des Strahlrohres kommen, ein Signal geben. Insgesamt senkt die erste Triggerstufe die Rate von $0.5 \mathrm{MHz}$ auf $75 \mathrm{kHz}$.

Triggerstufe T1: In der zweiten Triggerstufe T1 werden die Signale des BTRDs, des Wechselwirkungszählers und des Hodoskops 1 kombiniert. Hierbei wird auf eine Multiplizität von zwei geladenen Teilchen im Wechselwirkungszähler geschnitten (bei charm wird auf eine Multiplizität von größer als 3,5 getriggert). Weiterhin werden nur Ereignisse mit einem aktiven Hodoskopelement im zentralen $\left(\mathrm{H} 1^{0}\right)$ und einem im negativen Hodoskopbereich $\left(\mathrm{H}^{-}\right)$oder mit keinem aktiven Element im zentralen und mit zwei aktiven Elementen im negativen Bereich getriggert. Im positiven Bereich des Hodoskops $\left(\mathrm{H}^{+}\right)$darf keine Aktivität verzeichnet werden. Die Triggerbedingungen in dieser zweiten Triggerstufe lassen sich also wie folgt schreiben:

$$
\mathrm{T}_{1_{h e}}=[\mathrm{IC}=2] \cdot\left[\left(\left(\mathrm{H} 1^{-}=2\right) \cdot\left(\mathrm{H}^{0}=0\right)\right)+\left(\left(\mathrm{H} 1^{-}=1\right) \cdot\left(\mathrm{H} 1^{0}=1\right)\right)\right] \cdot\left(\mathrm{H} 1^{+}=0\right)
$$

Die Triggerlogik für die Selektion elastischer Streuung wurde während der Datennahme vom Februar bis September 1997 in mehreren Schritten optimiert. So war die zweite Triggerstufe nicht von Beginn an so implementiert, wie oben dargestellt. Die ersten Einstellungen waren $\mathrm{H}^{-}>0$ zusammen mit nicht $\mathrm{H}^{-}>1$, da ein Schnitt $\mathrm{H}^{-}>2$ nicht möglich war. 
Triggerstufe T2: Die dritte Triggerstufe fast die Ergebnisse der ersten beiden Stufen zusammen und erlaubt die Einbindung weiterer Informationen von Detektoren aus dem Bereich hinter dem Hodoskop 2, wie z.B. im Primakoff-Trigger die Energiesumme des dritten elektromagnetischen Kalorimeters. Im Hadron-Elektron-Trigger (h-e Trigger) wird diese Möglichkeit nicht genutzt.

Tabelle 3.4: Die Zeitpunkte der verschiedenen Trigger-Updates.

\begin{tabular}{|l|c|}
\hline Triggerversion & Runperiode \\
\hline 1ter h-e Trigger & $6822-7018$ \\
V5 Schwelle erniedrigt & $7019-9047$ \\
neue IC Schwellen & $9048-9098$ \\
2te h-e Trigger-Version & $9099-9653$ \\
online Filter & $9654-10061$ \\
IC Spannung erniedrigt & $10062-10127$ \\
neue IC Schwellen & $10128-10385$ \\
2te online Filter-Version & $10386-10858$ \\
\hline
\end{tabular}

Software-Trigger: Ab dem Run 9653 wird im Hadron-Elektron-Trigger die Möglichkeit eines online Software-Filters als vierte Triggerstufe genutzt. Dieser Software-Filter prüft die Ereignisse, welche die ersten drei Triggerstufen passiert haben auf folgende Eigenschaften:

- wenigstens ein Strahlsegment,

- nicht mehr als sechs Strahlsegmente,

- eine maximale Anzahl von Hits in den Strahl-, Vertex- und M2-Spektrometer (200, 1000 und 200), sowie maximal 1000 SVX words,

- keine positiv gekrümmten Spuren im M2-Spektrometer.

Diese erste Version des Online-Filters brachte eine Reduktion der Ereignisse um einen Faktor 1:1.2. In der zweiten Version des Online-Filters kamen zwei weitere Bedingungen hinzu:

- mindestens 20 Hits in den M2 PWCs,

- wenigstens eine negativ gekrümmte Spur im M2-Spektrometer.

Mit diesen Erweiterungen steigerte sich der Reduktionsfaktor auf 1:1.7.

Tabelle 3.4 gibt eine Übersicht über die verwendeten Triggerversionen. 


\section{Hodoskop- und Triggereffizienz}

Das Hodoskop 1 und der h-e Trigger sind eng verknüpft und ein genaues Verständnis dieser beiden ist für die Analyse der elastischen Streudaten unerläßlich, da beide großen Einfluß auf die Datennahme ausüben.

Ein Blick auf die $x$-Position der Pionkandidaten am Hodoskop 1 (Abb. 4.1) spiegelt deutliche die Struktur des Hodoskops wieder. Neben tiefen Einschnitten an den Positionen, an den zwei Hodoskopelemente zusammenstoßen, sind zwei deutliche Sprünge im Spektrum an den Übergängen zwischen dem negativen, zentralen und positiven Bereich des Hodoskops sichtbar. Bei genauer Betrachtung fällt weiterhin auf, dass das Hodoskopelement 17 an der Position -0.4--0.9 cm unter der Einhüllenden der Verteilung zurück bleibt und damit eine geringer Effizienz als die benachbarten Elemente zu haben scheint ${ }^{27}$.

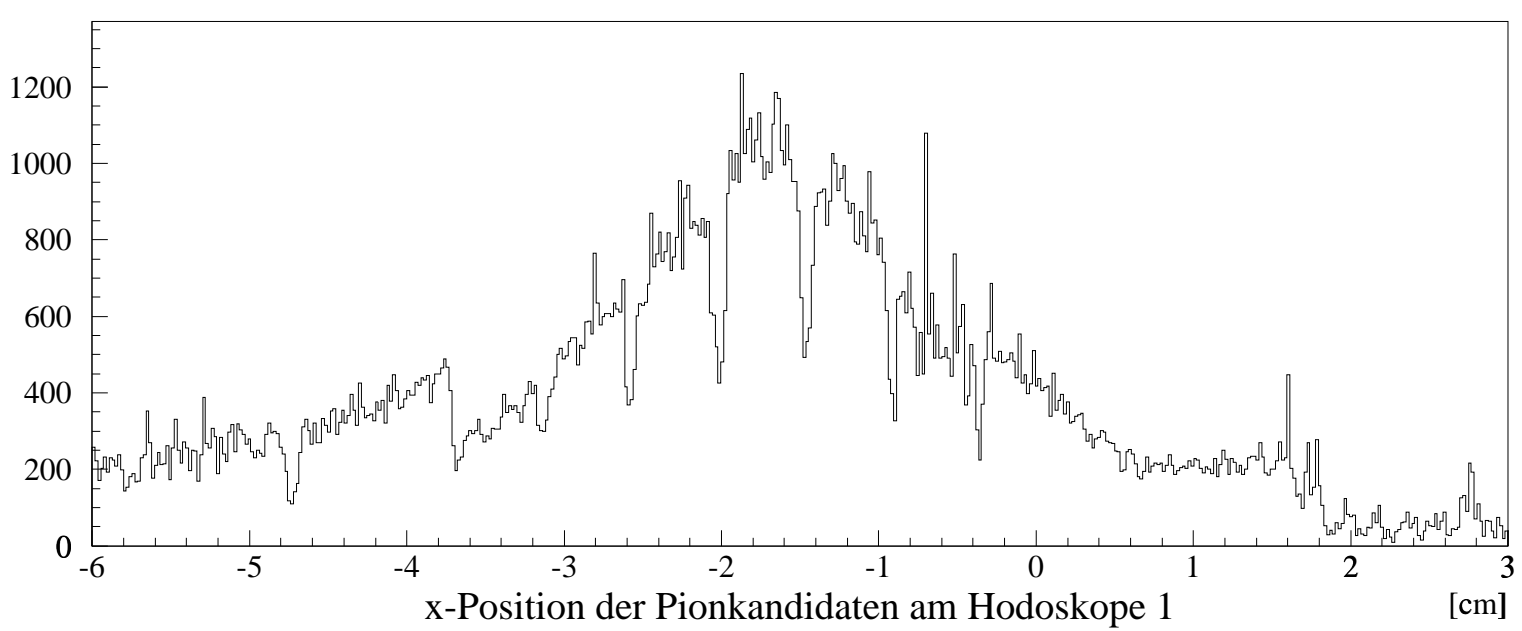

Abbildung 4.1: $x$-Positionen der Pionkandidaten am Hodoskop 1. Neben tiefen Einschnitten in der Verteilung an den Lücken des Hodoskop 1 sind auch deutliche Sprünge an den Übergängen von zentralen Bereich des Hodoskops zum negativen (bei $x=-3.7$ $\mathrm{cm}$ ) und zum positiven Bereich (bei $x=1.7 \mathrm{~cm}$ ) sichtbar.

Im folgenden Kapitel wird die Analyse der Position und Effizienz der einzelnen Hodoskopelemente dargelegt. Weiterhin wird der, sich aus den Eigenschaften des Hodoskops ergebende, $Q^{2}$-abhängige Anteil der Effizienz der T1-Stufe des Triggers ermittelt, welche fortan als Triggereffizienz bezeichnet wird. Dies ist in sofern gerechtfertigt, da die T0-Stufe in der Regel mit der Strahldefinition gleichgesetzt wird. Für diese T0Stufe, die weitgehend vom V5 Zähler dominiert wird, ließe sich zwar prinzipiell eine Effizienz definieren und bestimmen, diese sollte aber keinen Einfluß das $Q^{2}$-Spektrum haben und wäre damit für Untersuchungen von Formfaktoren irrelevant.

\footnotetext{
${ }^{27}$ Die Ursache liegt in diesem Fall allerdings nicht in der Effizienz sondern in einem vor diesem Element verlaufenden Stromkabel
} 


\subsection{Die Hodoskopanalyse}

Die Analyse des Hodoskop ist zuerst auf Primakoffdaten erfolgt, da diese sowohl über eine angemessene Multiplizität verfügen, als auch eine ausreichende Ausleuchtung des gesamten Hodoskops bieten. Die Multiplizität der Spuren im M2-Spektrometer sollte nicht zu groß sein, da sonst eine eindeutige Zuordnung von einzelnen Spuren zu den Hodoskopelementen nicht mehr möglich ist. Dies gilt insbesondere im zentralen Bereich des Hodoskops, welches in der Regel von den meisten Spuren getroffen wird. Als günstig erweist sich hierbei eine Multiplizität von bis zu vier Spuren und deswegen wurde bei der Analyse der Daten die Zahl der Spuren auf maximal vier beschränkt.

Bei der Analyse der Daten werden nur Spuren mit M2-Segment und zugeordnetem Vertex-Segment an der $z$-Position des Hodoskop 1 ausgewertet und mit den Aktivitäten der getroffenen Hodoskopelemente verglichen. Dabei werden nur solche Spuren berücksichtigt, auf die eine der folgenden Bedingungen zutrifft:

- Das Hodoskopelement, welches von der Spur getroffen wurde, hat gefeuert (Positionierung und Effizienz).

- Das Hodoskopelement zeigt keine Aktivität, aber ein direkt benachbartes Element hat gefeuert und in dessen Nachbarschaft sind keine weiteren Spuren detektiert worden (Positionierung).

- Weder das getroffene Element noch ein direkt benachbartes Element hat gefeuert und es sind keine weiteren Spuren in diesem Bereich des Hodoskops (Effizienz).

Die einzelnen Bedingungen dienen der Positionsmessung, Punkt 1 und 2, und oder der Effizienzbestimmung, Punkt 1 und 3.

\subsubsection{Position der Hodoskopelemente}

Teilt man die $x$-Positionsverteilung der Spuren mit Aktivität in einem bestimmten Element durch die zugrundeliegende Verteilung aller $x$-Positionen, so erhält man eine Kurve der Effizienz für das betreffende Element (Abb. 4.2). Fittet man an diese Kurve eine Rechteckfunktion aus zwei Stufenfunktionen, die jeweils mit einer Gaußkurve der mittleren Breite der Ortsauflösung gefaltet wurde, so kann man die Position der einzelnen Elemente bestimmen. Dabei wird der Abstand der beiden Stufenfunktionen und damit die Breite des Rechtecks, bzw. der einzelnen Hodoskopelemente fest vorgegeben, da die Produktion von Szintiallatoren einheitlicher Breite, auf einer zu betrachtenden Skala von ca. $0.1 \mathrm{~mm}$, technisch kein Problem darstellt.

Die Genauigkeit, mit der die $x$-Positionen der Hodoskop 1 Elemente vermessen wird, ist wegen der erreichbaren guten Statistik durch die Ortsauflösung der Projektion der Spuren begrenzt, welche unter Berücksichtigung der Fehlerfortpflanzungen von der Größenordnung $\pm 0.2 \mathrm{~mm}$ ist.

Diese Methode setzt voraus, dass das Koordinatensystem des Hodoskops nicht gegen das des Experiments verdreht ist, da ansonsten die Aufweichung der Ränder der Rechteckfunktion größer wird. Tatsächlich läßt sich eine leichte Verdrehung des Hodoskops von ca. 10 mrad im Gegenuhrzeigersinn feststellen (Abb. 4.3). 

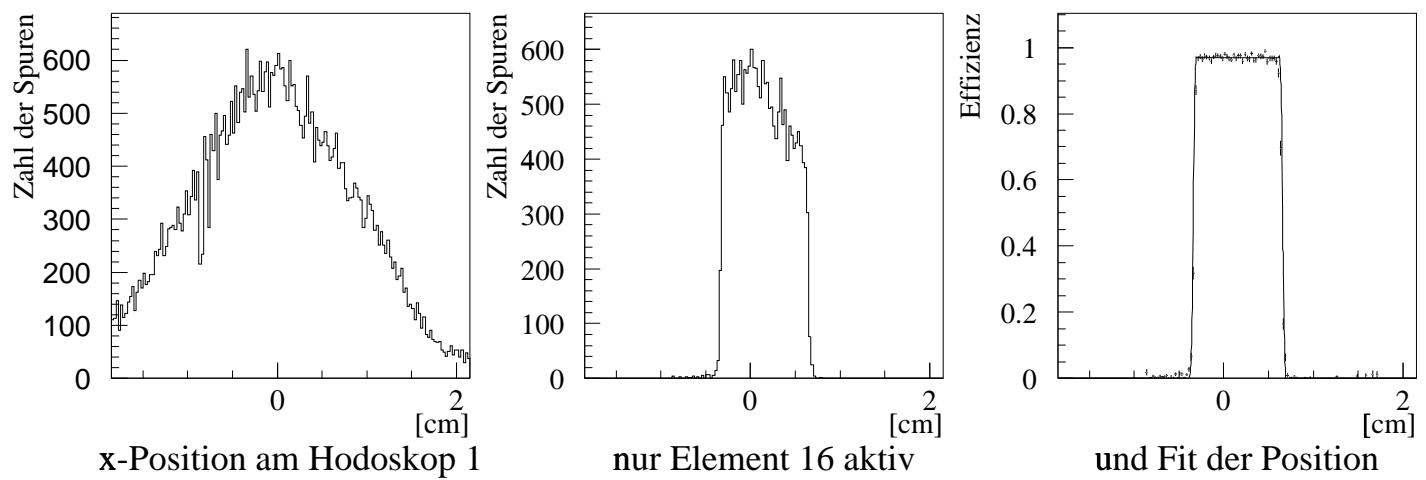

Abbildung 4.2: $x$-Positionsvermessung des Hodoskop 1 am Beispiel des Elements 16. Aus der $x$-Positionsverteilung der Spuren im M2-Spektrometer mit gelinktem VertexSegment (links), bei denen eine eindeutige Zuordnung möglich ist (vgl. Text), werden die Spuren mit Aktivität im Bereich des zu untersuchenden Element herausgeschnitten (Mitte) und diese Verteilung durch das Ausgangsspektrum dividiert (rechts). Aus der dabei erhaltenen Verteilung fittet man die Position mit einer Rechteckfunktion aus zwei Stufenfunktioen, die mit der Gaußkurve der mittleren Breite der Ortsauflösung gefaltet wurde.

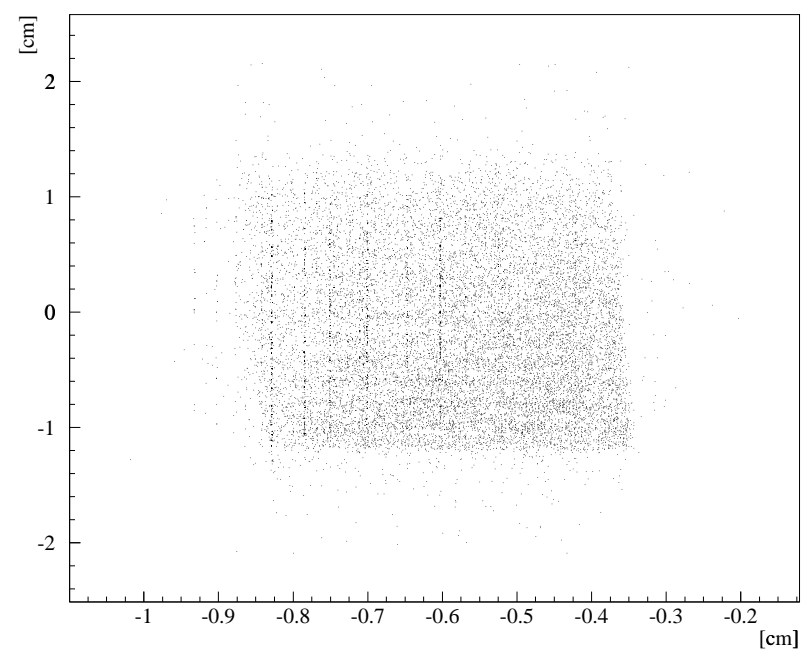

Abbildung 4.3: $y$-gegen $x$-Position der Spuren am Element 17 des Hodoskops 1. Eine leichte Kippung im Gegenuhrzeigersinn ist erkennbar.

Die Position der zentralen Hodoskopelement wird auf zeitliche Variationen hin untersucht, indem die Analyse für Daten unterschiedlicher Zeiträume wiederholt wird. Hierfür wurden die Primakoffruns 9178, 9900 und 10797 herangezogen, welche den Zeitraum von Mai bis August 1997 abdecken. Die hierbei ermittelten Werte stimmen innerhalb der Fehler überein. Dies ermöglicht die Statistik für die äußeren Elemente durch die gemeinsame Auswertung aller untersuchten Runs zu erhöhen. 


\subsubsection{Effizienz der Hodoskopelemente}

Die Primakoffdaten eignen sich nicht zur Bestimmung der Effizienz der einzelnen Hodoskopelemente, da bei deren Datennahme das Hodoskop 1 aktiv im Trigger verwendet wird. Deswegen wurde die Analyse der Effizienzen mit den Daten der totalen Wechselwirkungsquerschnittsmessung durchgeführt, da bei deren Datennahmen das Hodoskop 1 nicht im Trigger verwenden wurde und da diese ebenfalls eine gute Ausleuchtung des Hodoskops aufweisen.

Für die Effizienzbestimmung wird die Zahl der Spuren mit Aktivität in den einzelnen Hodoskopelementen durch die Gesamtzahl der Spuren in diesem geteilt (Abb. 4.4). Um Randeffekte auszuschließen werden dabei nur Spuren im zentralen Bereich der einzelnen Elemente berücksichtigt. Die resultierenden Effizienzen liegen für die meisten Elemente bei rund $94 \%$. Die Fehler der Effizienzmessung werden durch die Quantile $\lambda_{p}(k)$ der zugehörigen Poission-Verteilung bestimmt. Um eine Abschätzung des Untergrunds zu erhalten, wurde zu allen Spuren ein Offset von $6 \mathrm{~cm}$ hinzuaddiert und die Effizienzanalyse wiederholt. Die dabei erhaltene Zahl an zufälligen Aktivitäten liegt im Bereich von 1-3\%, wobei deren Zahl im zentralen, strahlnahen Bereich des Hodoskops höher liegt, als an den äußeren Rändern.
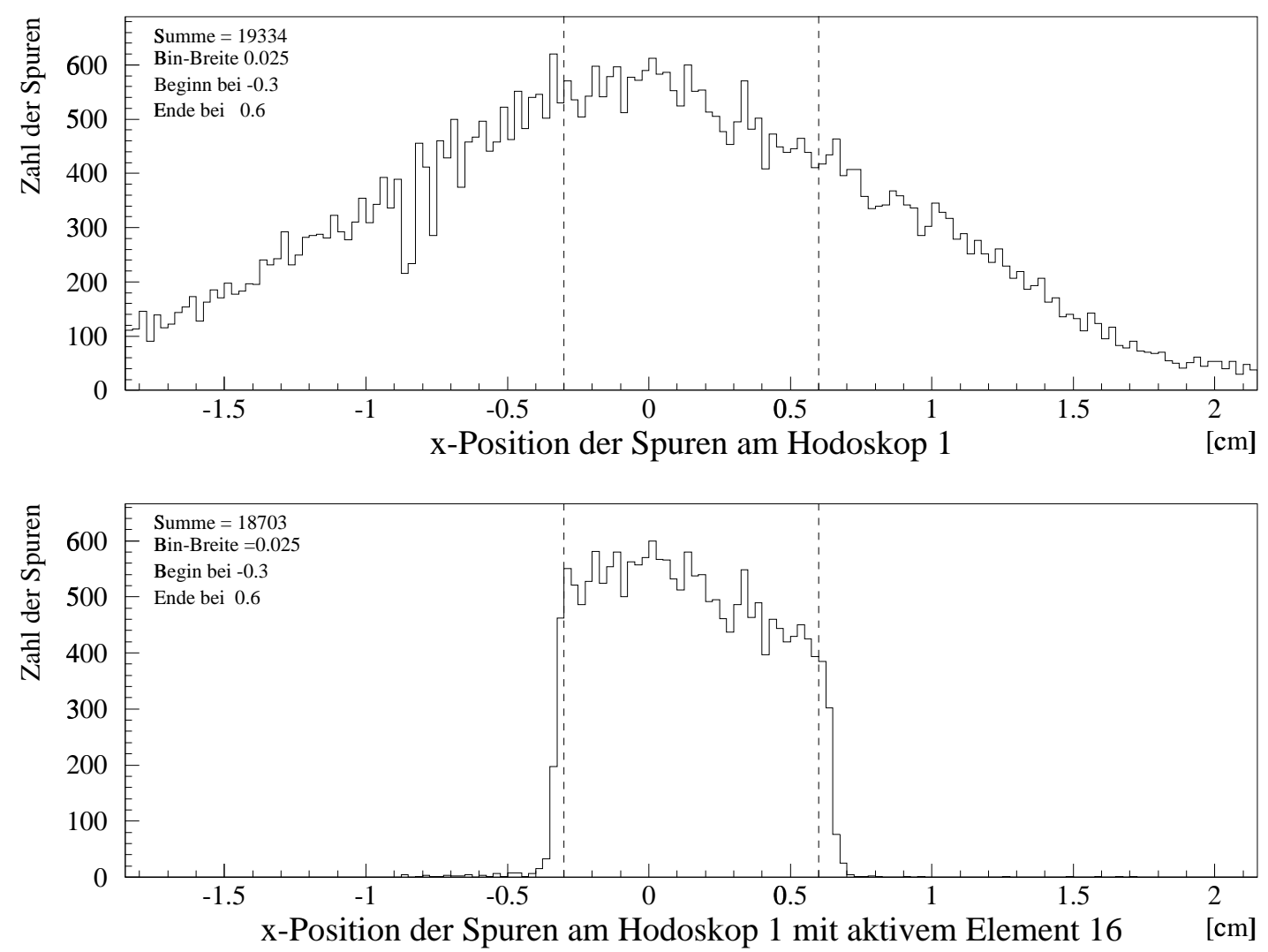

Abbildung 4.4: Effizienzmessung der Hodoskop 1 Elemente verfolgt über die Division der Zahl der Spuren, die durch den zentralen Bereich eines Hodoskopelements gehen (oben) und die Zahl der Spuren, die dabei auch Aktivität im diesem Bereich auslösen (unten). Zur Berechnung der Fehler werden die Quantile numerisch errechnet.

Die Effizienz der zentralen Hodoskopelemente ist mit Kalibrationsdaten auf etwaige 
zeitliche Abhängigkeiten untersuchen worden. Dabei zeigten diese innerhalb der Fehler für die zentralen Elemente keine zeitlichen Abhängigkeiten. Eine Untersuchung der äußeren Elemente auf zeitliche Varianzen ist nur schwer realisierbar, da die Kalibrationsdaten praktische keine Spuren in diesen Elemente aufweisen und da die Daten der totalen Wirkungsquerschnittsmessung innerhalb eines kurzen Zeitintervalls aufgezeichnet wurden.

Die Effizienzen und Positionen der einzelnen Hodoskopelemete sind in Anhang D aufgeführt.

\subsection{Die Triggereffizienz und Akzeptanzstudien}

Die Triggerlogik der T1-Stufe verbietet mehr als ein Teilchen in zentralen Bereich des Hodoskop 1 (vgl. Abschn. 3.4). Diese Einschränkung schneidet allerdings in das elastische Pion-Elektron-Streusignal, weil Ereignisse ab einem Viererimpulsübertrag von ca. $0.1 \mathrm{GeV}^{2} / c^{2}$ mit zunehmender Wahrscheinlichkeit von Trigger verworfen werden, da die Elektronen dann zusammen mit den Pionen den zentralen Bereich des Hodoskop 1 durchqueren. Abbildung 4.5 zeigt den Impulsübertrag gegenüber der Position des Elektrons (links) und des Pions (rechts) am Hodoskop 1, wie sie sich aus Simulationen ergeben.
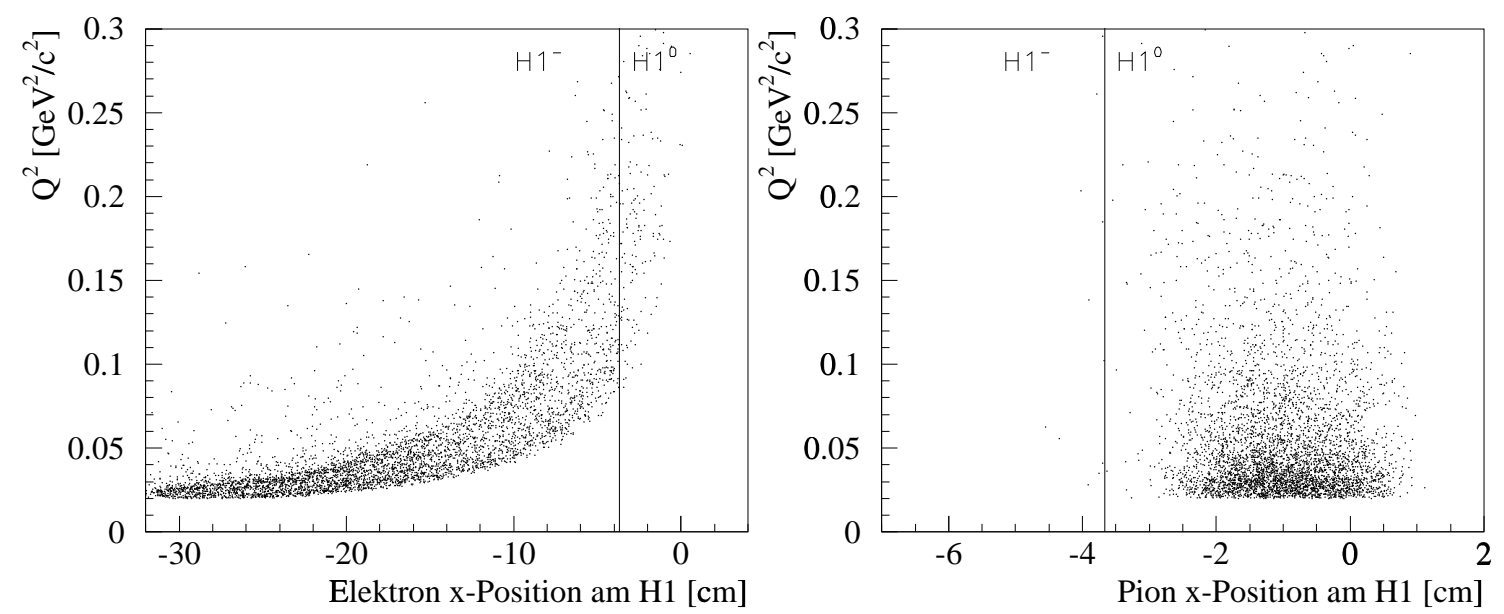

Abbildung 4.5: Der Impulsübertrag gegenüber der Elektron (links) und der Pion (rechts) $x$-Position am Hodoskop 1 für simulierte Ereignisse (GEANT). Die Grenze zwischen der negativen $\left(\mathrm{H}^{-}\right)$und der zentralen Bereich $\left(\mathrm{H}^{0}\right)$ des Hodoskops ist eingezeichnet.

Da das gemessene $\mathrm{Q}^{2}$-Spektrum mit dem Inversen der Akzeptanz des Spektrometers multipliziert werden muß, um Informationen über den mittleren quadratischen Ladungsradius $\mathrm{zu}$ erhalten, hat eine Akzeptanzfunktion, die keinen flachen Verlauf zeigt, einen großen Einfluß auf die Steigung des $Q^{2}$-Spektrums und damit auf den zu messenden Radius. Die obige Eigenschaft der T1-Triggerstufe, zeigt, das die Triggerakzeptanz ab einem Impulsüberträg von mehr als $0.1 \mathrm{GeV}^{2} / c^{2}$ abfällt und somit das $\mathrm{Q}^{2}$-Spektrum verändert.

Im folgenden wird kurz das verwendete Simulationswerkzeug vorgestellt, mit dem die Triggereffizienz und im späteren Teil die Akzeptanzstudien untersucht werden. 


\subsubsection{GEANT Simulation}

Ein wichtiges Hilfsmittel zum Verständnis der Vorgänge im Experiment sind Simulationen des Detektorverhaltens. Da dieses aufgrund der statistischen Natur der Wechselwirkungen von Teilchen mit Materie nicht Ereignis für Ereignis geschehen kann, müssen umfangreiche Datensätze simuliert werden, die dann zum Vergleich mit den real gemessenen Daten dienen. Hierzu wird das GEANT ${ }^{28}$-Paket, welche den Durchgang elementarer Teilchen durch Materie simuliert, verwendet. Seine Hauptanwendung findet GEANT dabei in der Simulation der Antwortfunktion von Detektoren. Dabei erlaubt GEANT

- die Beschreibung des experimentellen Aufbaus durch Struktur- und Geometriebeschreibungen der einzelnen Detektorelemente, wobei die Eigenschaften der verwendeten Materialien berücksichtigt werden,

- die Verarbeitung von Monte Carlo generierten Ereignissen,

- die Simulation des Transportes von Teilchen durch den experimentellen Aufbau unter Berücksichtigung geometrischer Gegebenheiten, sowie physikalischer Effekte aufgrund der Natur des Teilchens selbst als auch aufgrund seiner Wechselwirkungen mit Materie und elektromagnetischen Feldern,

- die Aufzeichnung von Teilchenspuren als auch der entsprechenden Detektorantworten,

- die graphische Darstellung des experimentellen Aufbaus und der Trajektorien von Teilchen.

Der Funktionsweise des GEANT-Pakets liegt folgende Strategie zugrunde:

Ausgehend von dem einlaufenden Teilchen, dessen Impuls aus dem gemessenen Strahlimpulsspektrum erwürfelt wird, werden hierzu die Wechselwirkungen gemäß der physikalischen Wahrscheinlichkeiten zufällig generiert, wobei die Wahrscheinlichkeit einer elastischen Streuung mittels eines speziellen Generators elastischer Ereignisse auf eins gesetzt wird und somit nur der Wechselwirkungspunkt gemäß der Materialverteilung bestimmt werden muß. Der Viererimpulsübertrag $\mathrm{Q}^{2}$ wird aus dem kinematisch zugänglichen Intervall per Zufall ausgewählt und nach der Verwerfungsmethode von Neumann an den Wechselwirkungsquerschnitt angepaßt. Die Reaktionspartner werden daraufhin durch das gesamte Spektrometer geführt, wobei auch mögliche sekundäre Wechselwirkungen (elastische, wie inelastische Streuungen, ČerenkovStrahlung, Bremsstrahlungsprozesse und die Konversion von Photonen dieser Prozesse in Elektron-Positron-Paare, usw.) erzeugt werden. Hierfür liegen in einer Datenbank die Information über sämtliche Materialverteilungen vor, so dass diese sekundären Wechselwirkungen gemäß der bekannten physikalischen Gesetze der Wechselwirkungen von Strahlung mit Materie generiert werden können. Für alle so produzierten Teilchen werden die Detektorantworten entsprechend der gemessenen Effizienzen generiert. Diese werden daraufhin im SELEX-Datenformat gespeichert, wobei ein zusätzlicher Datenblock über die verwendeten Spurelemente und andere Parameter Auskunft gibt,

\footnotetext{
${ }^{28}$ Das Akronym GEANT stammt von geometry and tracking.
} 

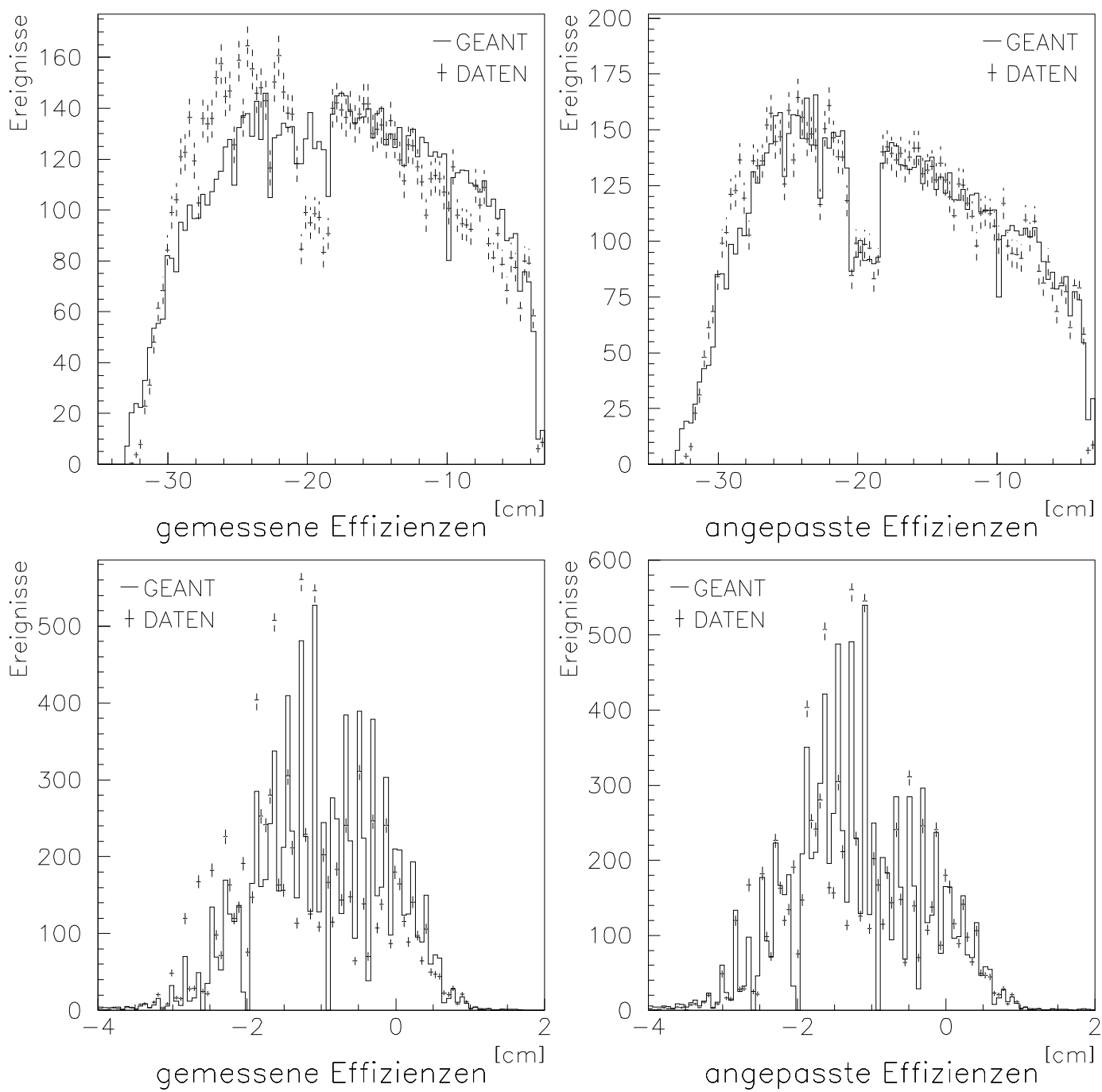

Abbildung 4.6: Die $x$-Positionsspektrum der Elektronen (oben) und Pionen (unten) an Hodoskop $1 \mathrm{im}$ Vergleich zwischen GEANT und Daten für die gemessenen Hodoskopeffizienzen (jeweils links) und für die an die Verteilung der Daten angepaßten Effizienzen (jeweils rechts).

und somit einen Vergleich der später rekonstruierten Werte mit den zugrundeliegenden ermöglicht.

\subsubsection{Der $\mathrm{Q}^{2}$-abhängige Anteil der Triggereffizienz}

Das Studium der Triggereffekte beschränkt sich auf den Einfluß der T1-Stufe. Neben Verlusten aufgrund der Triggerlogik der T1-Stufe, wenn beide Teilchen den zentralen Bereich des Hodoskops passieren (vgl. Einleitung des Abschn. 4.2 und Abb. 4.5), gehen in der T1-Stufe Ereignisse aufgrund von Hodoskop 1 Ineffizienzen verloren, wenn eines der getroffenen Hodoskopelemente nicht feuert, oder wenn eines der Teilchen eine Lücke passiert. Die Triggerwahrscheinlichkeit läßt sich mit der Kenntnis der Effizienzen der einzelnen Hodoskopelemente simulieren. Hierbei werden die Spuren der 
gestreuten Teilchen an der Stelle des Hodoskop 1 ausgewertet und dem entsprechenden Element, bzw. der entsprechenden Lücke, zugeordnet. Hierbei läßt sich zusätzlich das $x$-Positionsspektrum der simulierten Spuren am Hodoskop 1, so man sie mit den Effizienzen des jeweiligen Module gewichtet, mit dem entsprechenden Spektrum aus den Daten vergleichen. Abbildung 4.6 zeigt diesen Vergleich, wobei eine deutliche Diskrepanz zwischen den simulierten Daten und den gemessenen Daten auffällt (linke Seite der Abb. 4.6). Dieser Diskrepanz kann benutzt werden um die Effizienzen neu anzupassen, um so eine Übereinstimmung der Spektren zu erzwingen. Die so gewonnenen Effizienzen (vgl. Tab. D.2) zeigen eine interessante Systematik, bei der die Effizienzen der einzelnen Elemente, in beiden mit dieser Methode zugänglichen Hodoskopbereichen, von außen nach innen hin abnehmen. Die Ursache sollte in der Triggerelektronik liegen, da physikalische Gründe für eine solche Systematik nur schwerlich verantwortlich sein können. Eine Möglichkeit hierbei könnte in Laufzeitunterschieden innerhalb der einzelnen Stufen der Signalverarbeitung innerhalb des Triggers liegen.

Um nun den $\mathrm{Q}^{2}$-abhängigen Anteil der Triggereffizienz zu bestimmen, wertet man die Hodoskoptreffer mit der Triggerlogik aus und gewichtet bei positiver Triggerentscheidung, den zu diesem Ereignis gehörigen Impulsübertrag in der $\mathrm{Q}^{2}$-Verteilung mit dem Produkt der Ansprechwahrscheinlichkeiten der getroffenen Hodoskopelemente. Das so erhaltene $\mathrm{Q}^{2}$-Spektrum wird durch das Spektrum der Eingangsdaten (alle Ereignisse mit den Gewicht von 1.) geteilt, um so die Triggereffizienz als Funktion des Impulsübertrags zu erhalten (Abb. 4.7).
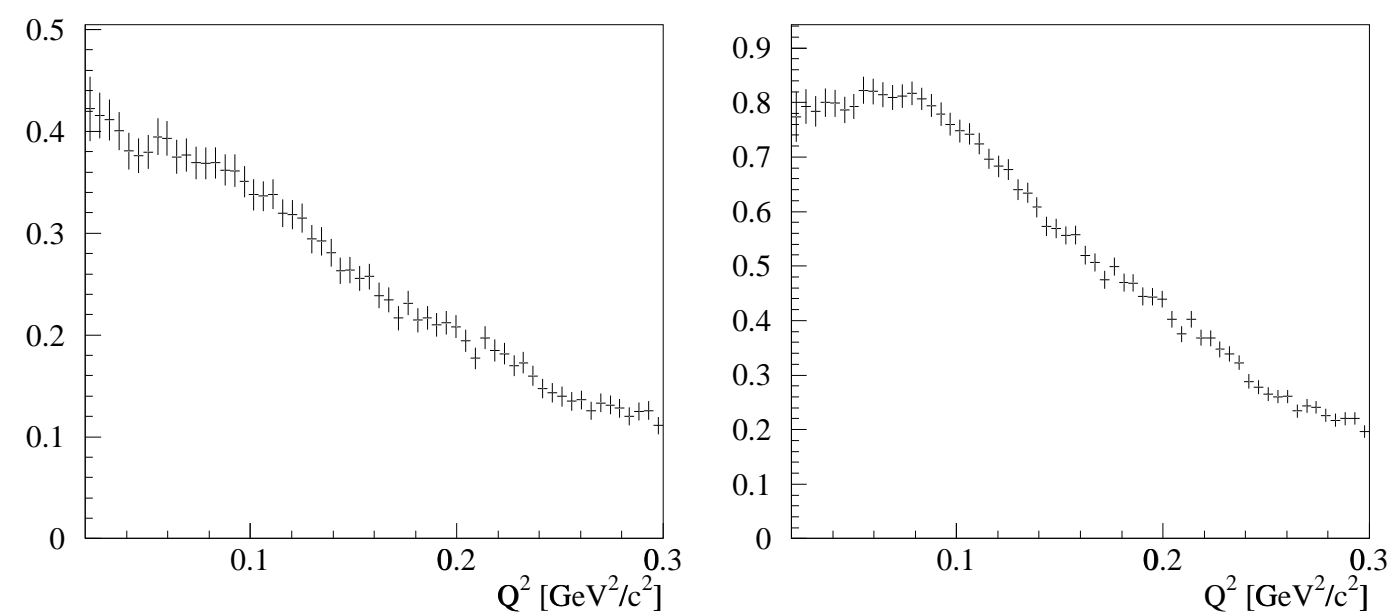

Abbildung 4.7: Die Triggerakzeptanz für angepaßte (links) und gemessene (rechts) Hodoskopeffizienzen, wie sie sich aus simulierten Ereignissen in einem $\mathrm{Q}^{2}$-Intervall von 0.02 bis $0.3 \mathrm{GeV}^{2} / c^{2}$ ergibt. Das Niveau bei kleinen $\mathrm{Q}^{2}$ von rund $40 \%$ in linken und von $80 \%$ im rechten Bild ergibt sich weitgehend aus den Effizienzen die den einzelnen Hodoskopelementen zugewiesen ist. Diese liegt Schwanken bei den angepaßten Effizienzen zwischen 1.0 und 0.4, während sie bei den gemessenen Effizienzen fast alle um die 0.9 liegen. Der sich anschließende Abfall der Triggerakzeptanz ab einem $\mathrm{Q}^{2}$ von $0.1 \mathrm{GeV}^{2} / c^{2}$ wird durch die Triggerlogik hervorgerufen. 


\subsubsection{Akzeptanzstudien}

Die Akzeptanzfunktion, oder kurz die Akzeptanz, beschreibt die mittlere Wahrscheinlichkeit ein Ereignis nachzuweisen, wobei man in der Regel unterscheidet zwischen der geometrischen Akzeptanz, ausgehend von der endlichen Größe des Detektors, und der Nachweiswahrscheinlichkeit des Detektors, also seiner endlichen Effizienz. Weitere Effekte, die auf die Akzeptanzfunktion Einfluß nehmen, sind die Totzeit während der Auslese und Ineffizienzen in den online und offline Rekonstruktionen. In die Akzeptanzfunktion fließen folglich alle Effekte ein, die eine Abnahme der Ereigniszahl zur Folge haben.

Wenn $x=\left(x_{1}, x_{2}, \ldots x_{D}\right)$ die physikalischen Variablen sind, die ein Ereignis beschreiben, so wie z.B. bei der elastischen Streuung Impulse, Streuwinkel und Impulsübertrag, so folgen diese Variablen der Wahrscheinlichkeitsverteilung

$$
f(x) \mathrm{d}^{D} x=\frac{F(x) \mathrm{d}^{D} x}{\int_{\Omega} F(x) \mathrm{d}^{D} x} .
$$

Dabei ist $\Omega$ der erlaubte Bereich von $x$ und das Integral schließt die Summen über diskrete Variablen mit ein. Die nicht normalisierte Dichte $F(x)$ hängt von den experimentellen Gegebenheiten ab und ist proportional zum differentiellen Wirkungsquerschnitt. Für hinreichend kleine Bereiche des Phasenraums ist der Wirkungsquerschnitt nahezu konstant und fällt aus der normalisierten Wahrscheinlichkeitsdichte $f(x)$ heraus.

Mit der Nachweiswahrscheinlichkeit $\varepsilon(x)$ für ein Ereignis mit den physikalischen Variablen $x$, ergibt sich für die Akzeptanz $a$

$$
a=\int_{\Omega} \varepsilon(x) f(x) \mathrm{d}^{D} x
$$

Akzeptanzen werden üblicherweise durch Monte Carlo Methoden ermittelt. Simuliert man M (pseudo-)zufallsverteilte Ereignisse $x^{(1)}, x^{(2)}, \ldots x^{(m)}$, die der Wahrscheinlichkeitsverteilung $f(x) \mathrm{d}^{D} x$ folgen, so erhält man eine Monte Carlo Abschätzung $A$ für die Akzeptanz a

$$
A=\frac{\sum_{i=1}^{M} \varepsilon\left(x^{(i)}\right)}{M}
$$

mit einer abgeschätzten Varianz von

$$
(\Delta A)^{2}=\frac{\sum_{i=1}^{M} \varepsilon\left(x^{(i)}-A\right)^{2}}{M(M-1)} .
$$

Im folgenden wird die Akzeptanz als Funktion des Impulsübertrags untersucht, da diese die für den mittleren quadratischen Ladungsradius nötigen Informationen enthält. Dabei soll die Akzeptanz in zwei Teile aufgespalten werden, zum einen in die Rekonstruktionswahrscheinlichkeit als die Summe aus den Detektorineffizienzen und den Verlusten in der online und offline Rekonstruktion und zum anderen in die Triggerwahrscheinlichkeit. Hierzu wurde mit dem GEANT-Paket eine Simulation von 100000 Ereignissen der elastischen Pion-Elektron-Streuung für Pionen mit einem mittleren quadratischen Ladungsradius von $0.44 \mathrm{fm}^{2}$ im $\mathrm{Q}^{2}$-Bereich von $0.02-0.30 \mathrm{GeV}^{2} / c^{2}$ 
erstellt. Die in dieser Simulation erzeugte $\mathrm{Q}^{2}$-Verteilung ist in Abb. 4.8 (links) gezeigt. Zur Kontrolle der Simulation plottet man das $\mathrm{Q}^{2}$-Spektrum gewichtet mit dem Inversen des Wechselwirkungsquerschnittes für Pionen mit einem mittleren quadratischen Ladungsradius von $0.44 \mathrm{fm}^{2}$ (Abb. 4.8 rechts und Gl. 2.33). Dies muß eine innerhalb der statistischen Fluktuationen flache Verteilung ergeben.

Prozessiert man die in dieser Simulation erzeugten Daten mit SOAP, so erhält man ein von den Schnitten abhängiges $\mathrm{Q}^{2}$-Spektrum und somit auch eine von den Schnitten abhängige Akzeptanz. Im Abb. 4.9 ist das von SOAP rekonstruierte $\mathrm{Q}^{2}$-Spektrum und die sich aus diesem Spektrum ergebende Akzeptanzfunktion gezeigt. Als Schnitte werden die in Abschn. 5.2.4 definierten Schnitte der Datenanalyse benutzt. Die so erhaltene Akzeptanzfunktion beinhaltet noch keinerlei Effekte des Triggers.

Die Verluste in der Akzeptanz haben vorwiegend folgende Quellen.

- Bei ca. 6 \% der simulierten Ereignisse wird keine Elektron im M2-Spektrometer gefunden. Diese Verluste sind stark $\mathrm{Q}^{2}$-abhängig und sind vornehmlich für den starken Abfall der Akzeptanz bei kleinem $\mathrm{Q}^{2}$ unterhalb von $0.05 \mathrm{GeV}^{2} / c^{2}$ verantwortlich (Abb. 4.10 links).

- Bei ca. $15 \%$ der simulierten Ereignisse hat die gefundene Elektronspur kein Vertex-Segment, welche für das Auffinden eines Vertex von Nöten ist. Diese Verluste zeigen eine leichte und weitgehende lineare $\mathrm{Q}^{2}$-Abhängigkeit, der Form, dass bei kleinen $\mathrm{Q}^{2}$ etwa doppelt so viele Ereignisse verworfen werden, wie bei großem $\mathrm{Q}^{2}$ (Abb. 4.10 mitte).

- Bei ca. $8 \%$ der simulierten Ereignisse hat der gefundene Vertex kein auslaufendes Elektron. Diese Verluste zeigen ebenfalls eine leichte $\mathrm{Q}^{2}$-Abhängigkeit, welche allerdings mit $\mathrm{Q}^{2}$ steigt (Abb. 4.10 links).

- Die in den Bildunterschriften der Abb. 4.9 und 4.11 spezifizierten Schnitte führen
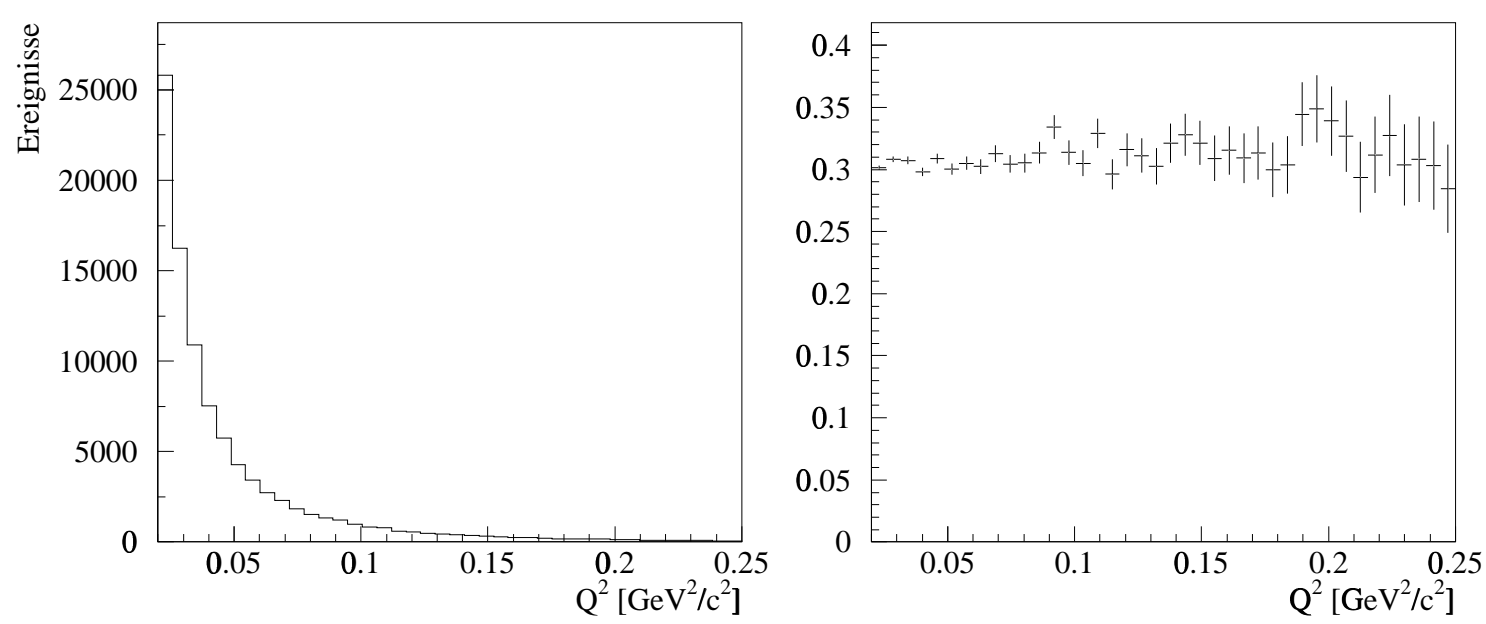

Abbildung 4.8: GEANT erzeugtes $\mathrm{Q}^{2}$-Spektrum für Pionen mit einem mittleren quadratischen Ladungsradius von $0.44 \mathrm{fm}^{2}$ (links) und selbiges gewichtet mit dem Inversen des Wirkungsquerschnitt unter Berücksichtigung des Radius (rechts), was erwartungsgemäß ein flache Akzeptanz ergibt. 

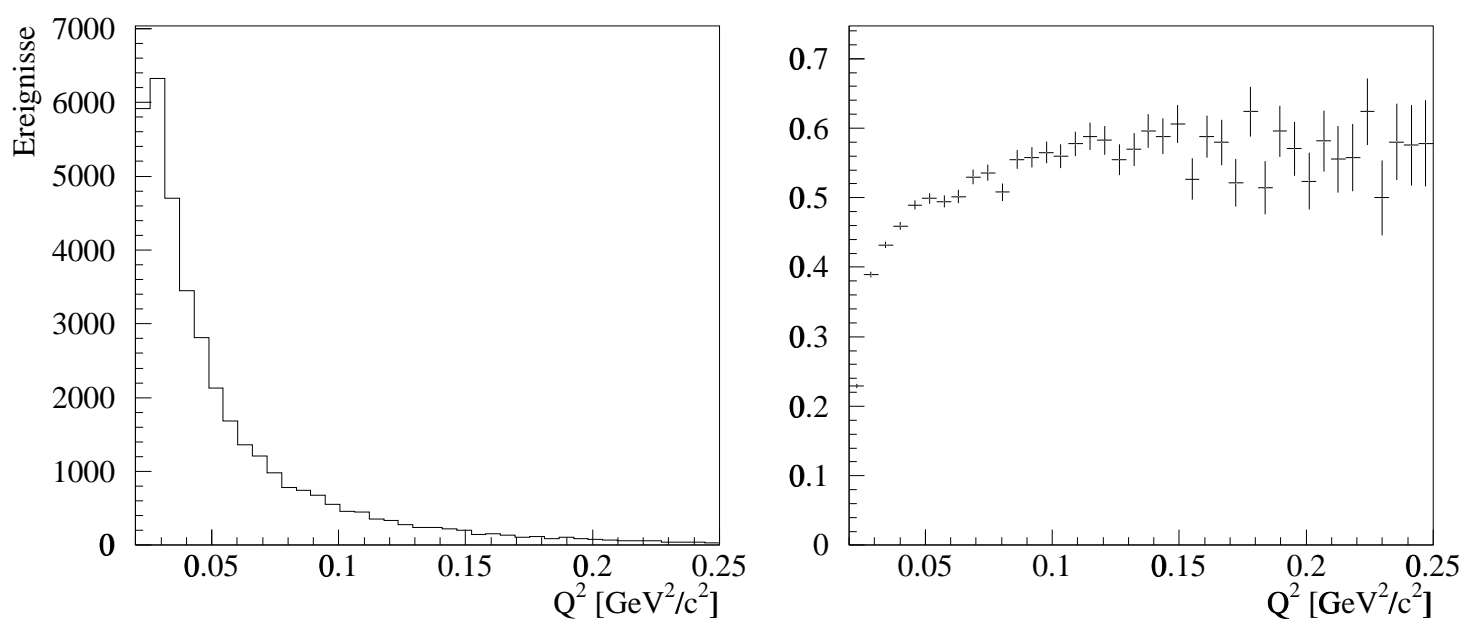

Abbildung 4.9: Soap rekonstruiertes $\mathrm{Q}^{2}$-Spektrum der GEANT Simulation (links) und dieses durch das von GEANT erzeugte Spektrum (Abb. 4.8 links) geteilt (rechts). Geschnitten wurde auf einen Elektronenpull von kleiner 3.0, eine Pionenpull von kleiner 3.5, einer Vertex-Elastizität kleiner als $30 \mathrm{GeV} / c$ und einem $\chi^{2}$ des Vertexfits von kleiner 5 .

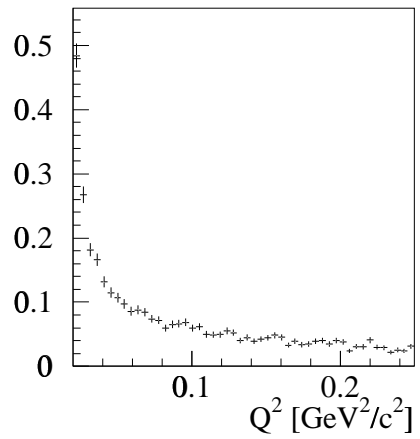

Kein Elektron,

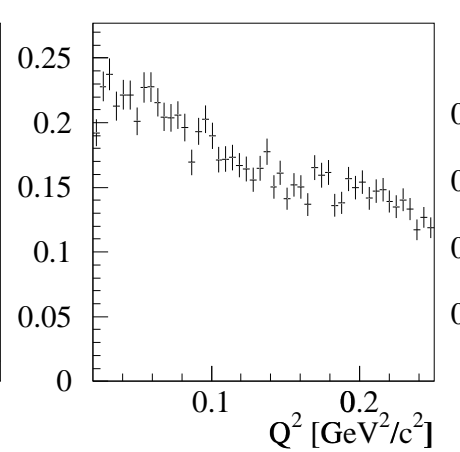

mit Vertex-Segment,

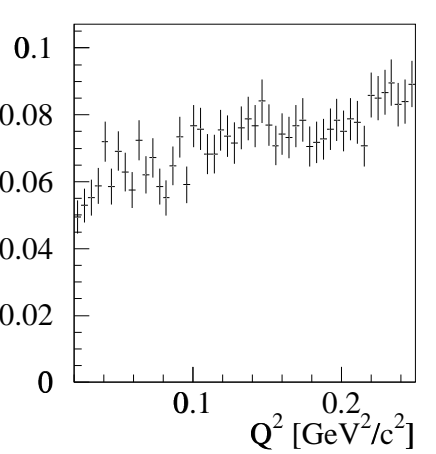

im Vertex gefunden

Abbildung 4.10: Anteile der verschiedenen Akzeptanzverluste, wie dadurch, dass kein Elektron gefunden wurde (links), dadurch dass kein Elektron mit Vertex-Segment gefunden wurde (mitte) und letztlich dadurch dass im Vertex keine Elektronspur gefunden wurde (rechts).

zu weiteren Verlusten der Größenordnung von ca. $8 \%$, zeigen aber unterhalb von $\mathrm{Q}^{2}=0.25 \mathrm{GeV}^{2} / c^{2}$ keine $\mathrm{Q}^{2}$-Abhängigkeit. Der Einfluß dieser Schnitte der Datenanalyse wird im Abschn. 5.4.1 diskutiert.

Gewichtet man die Ereignisse im rekonstruierten $\mathrm{Q}^{2}$-Spektrum mit der Triggerwahrscheinlichkeit aus Abschn. 4.2.2, so erhält man die Akzeptanz inklusive der Triggereffekte (Abb. 4.11).

Die Fehler der Akzeptanzfunktion, wie sie in den Abb. 4.8-4.11 dargestellt sind, skalieren mit dem Wirkungsquerschnitt. Um über den gesamten $\mathrm{Q}^{2}$-Bereich gleichmäßig große Fehler zu erhalten, berechnet man die Akzeptanz nicht mit der $\mathrm{Q}^{2}$-Verteilung der elastischen Streuung sondern mit einer flachen $Q^{2}$-Verteilung. 

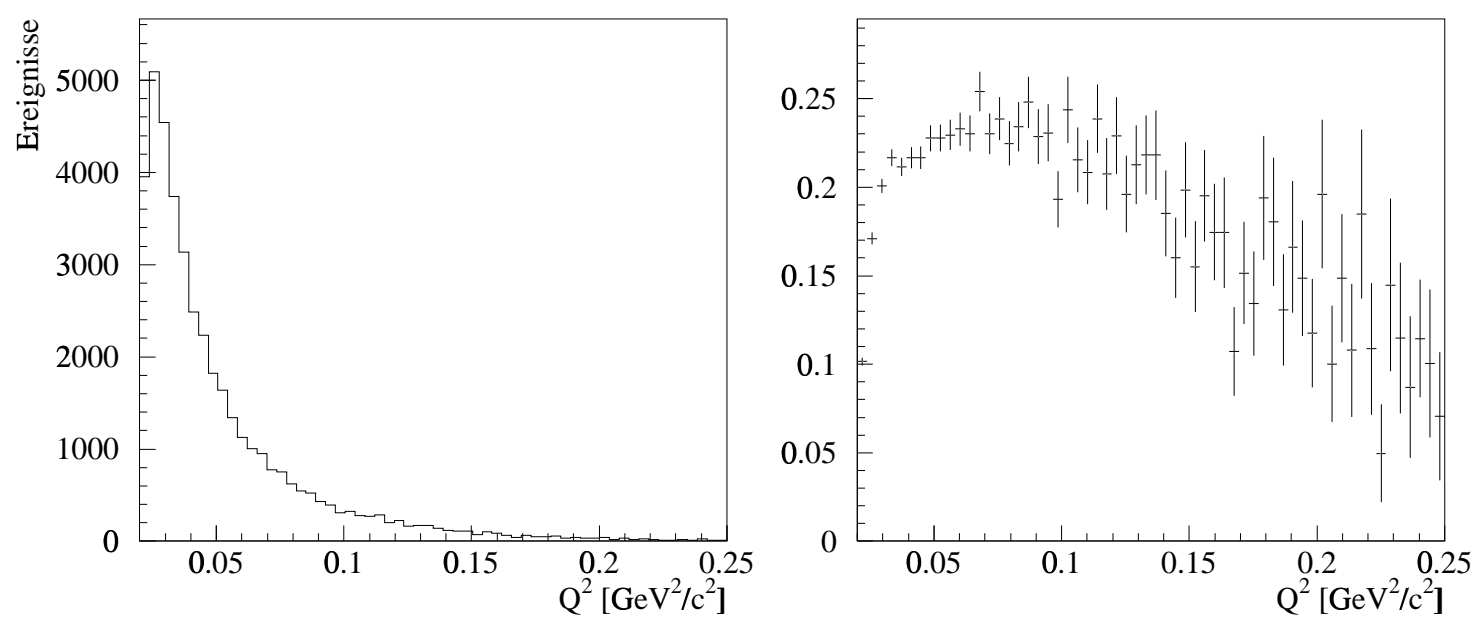

Abbildung 4.11: Mit der Triggerwahrscheinlichkeit (gemessene Effizienzen) gewichtetes von Soap rekonstruiertes $\mathrm{Q}^{2}$-Spektrum der GEANT Simulation (links) und dieses durch das von GEANT erzeugte Spektrum (Abb. 4.8 links) geteilt (rechts). Geschnitten wurde auf einen Elektronenpull von kleiner 3.0, eine Pionenpull von kleiner 3.5, einer Vertex-Elastizität kleiner als $30 \mathrm{GeV} / c$ und einem $\chi^{2}$ des Vertexfits von kleiner 5 . 


\section{Datenanalyse}

Aus den rund 215 Millionen Ereignissen, die während der Meßzeit bis zum September 1997 aufgezeichnet wurden, werden in mehreren Schritten ca. 24000 Ereignisse extrahiert, die zur Messung des mittleren quadratischen Ladungsradius genutzt werden. Die einzelnen Schritte dieser Auswahl und die Analyse der so gewonnenen Daten sind Gegenstand dieses Kapitels.

Im ersten Teil des folgenden Kapitel wird zuerst kurz auf die Datenreduktion eingegangen, bevor dann im zweiten Teil die einzelnen Schritte der Datenaufbereitung und die anschließende Datenanalyse vorgestellt werden. Den Abschluß bilden Studien der Abhängigkeit des gemessenen Radius von den einzelnen Schnitten und damit die Betrachtung der systematischen und statistischen Fehler.

\subsection{Datenreduktion}

Die Zahl der zu erwartenden elastischen Pion-Elektron-Streuereignisse $N_{\text {elast }}$ kann aus dem totalen Wechselwirkungsquerschnitt $\sigma_{\text {elast }}$ mit der Zahl der einlaufenden Strahlteilchen $N_{\text {Strahl }}$, der Akzeptanz $a$ und den Materialeigenschaften der Targets abgeschätzt werden über

$$
N_{\text {elast }}=N_{\text {Strahl }} \cdot a \cdot \sigma_{\text {elast }} \cdot \frac{Z}{A} \cdot N_{A} \cdot \rho \cdot l
$$

Zur Notation vergleiche Anhang C. Für das Produkt $\frac{Z}{A} \cdot N_{A} \cdot \rho \cdot l$ ergibt sich mit den Werten aus der Tab. 5.1 ein Wert von $1.2 \cdot 10^{24} / \mathrm{cm}^{2}$. Der Wechselwirkungsquerschnitt für elastische Streuung kann aus der Formel 2.33 durch Integration über den experimentell zugänglichen $\mathrm{Q}^{2}$-Bereich gewonnen werden. Für einen mittleren Strahlimpuls von $600 \mathrm{GeV} / c$ ist dies der Bereich von $[0.025-0.59] \mathrm{GeV}^{2} / c^{2}$, wobei sich die untere Grenze aus der Akzeptanz des SELEX-Spektrometers ergibt. Somit erhält man für Pionen mit einem mittleren quadratischen Ladungsradius von $0.44 \mathrm{fm}^{2}$

$$
\sigma_{\text {elast }}=\int_{0.025 \mathrm{GeV}^{2} / c^{2}}^{0.59 \mathrm{GeV}^{2} / c^{2}}\left(\frac{d \sigma}{d \mathrm{Q}^{2}}\right) d \mathrm{Q}^{2} \approx 8 \mu \text { barn } .
$$

Die Zahl der einlaufenden Strahlteilchen während der Run-Periode von 10000 bis 11000 mit aktivem h-e Trigger und gültiger Strahldefinition beläuft sich zu $1.36 \cdot 10^{11}$ Teilchen. Der Anteil an Pionen liegt bei ca. 50 \%. Die Akzeptanz a der Rekonstruktion und des Triggers ohne den Wechselwirkungszähler beträgt ca. 0.4 (vgl. Abschn. 4.2). Dies führt zu einem zu erwartenden Datensatz von $6.5 \cdot 10^{5}$ Ereignissen.

Die Datenreduktion erfolgt in drei Stufen, wobei das Konzept verfolgt wird, in der ersten Stufe nach Kriterien zu suchen, welche sich schnell prozessieren lassen und die auf einfachen Detektorelementen beruhen, um dann in der zweiten Stufe die so selektierten Ereignisse anhand von aufwendigeren Kriterien genauer zu untersuchen. Die Kriterien werden hierbei so offen wie möglich gehalten, um so möglichst wenig elastische Streuereignisse zu verlieren. Zur Datenreduktion wird das SOAP ${ }^{29}$-Paket verwendet.

\footnotetext{
${ }^{29} \underline{\mathrm{SELEX}}$ off-line analysis package
} 


\begin{tabular}{|c|c|c|c|c|}
\hline $\begin{array}{c}\text { Target } \\
\text { Nummer }\end{array}$ & Material & $\begin{array}{c}l \\
{[\mathrm{~cm}]}\end{array}$ & $\begin{array}{c}\rho \\
{\left[\mathrm{g} / \mathrm{cm}^{3}\right]}\end{array}$ & $\begin{array}{c}Z / A \\
{[\mathrm{~mol} / \mathrm{g}]}\end{array}$ \\
\hline \hline 1 & $\mathrm{Cu}$ & 0.16 & 8.96 & 0.456 \\
2 & $\mathrm{Cu}$ & 0.1016 & 8.96 & 0.456 \\
3 & $\mathrm{C}$ & 0.22 & 3.23 & 0.500 \\
4 & $\mathrm{C}$ & 0.22 & 3.23 & 0.500 \\
5 & $\mathrm{C}$ & 0.22 & 3.23 & 0.500 \\
\hline
\end{tabular}

Tabelle 5.1: Kenngrößen der Targets, wie die Länge $l$ in Strahlrichtung, die Dichte $\rho$, sowie Kernladungszahl $Z$ und die Atommassenzahl $A$.

In SOAP sind die Routinen zur Rohdatenaufbereitung zusammengefaßt. Hierzu zählen Routinen zum Entpacken der Rohdaten und zur Spurrekonstruktion in den einzelnen Spektrometern, sowie das Zusammenfügen einzelner Teilspuren (den sog. tsegs $^{30}$ ) aus verschiedenen Spektrometern zu den eigentlichen Spuren (tracks). Weiterhin verfügt SOAP über Routinen zur Vertexsuche. SOAP stellt alle Informationen der Rohdaten, der Spurrekonstruktion und der Vertexsuche dem Benutzer zur weiteren Analyse zur Verfügung. Hierzu werden benutzerdefinierte Routinen dem SOAPProgrammcode hinzugefügt. Weiterhin lassen sich vom Benutzer Parameter für alle Schritte der Datenaufbereitung vorgeben, wie z.B. welche Detektorinformationen und Kombinationen zur Spurrekonstruktion verwendet werden sollen, die maximal erlaubten $\chi^{2}$ der Spurrekonstruktion oder die Parameter der Teilchenidentifikation des ETRD und BTRD, etc.

Die drei Stufen der Datenreduktion sind:

Strip 1: Strip 1 prüft die Ereignisse vornehmlich auf die Existenz einer Elektronenspur im M2-Spektrometer. Dabei werden Ereignisse verworfen, die

- kein M2-Segment aufweisen,

- in denen kein Elektron gefunden wurde,

- in denen kein Elektron mit einem zugeordneten Vertex-Segment gefunden wurde,

- die kein Elektron mit einem Impuls von weniger als $500 \mathrm{GeV} / c$ aufweisen.

Die Identifizierung des Elektrons erfolgt hierbei über den ETRD. Dabei wird gefordert, dass für ein Elektron wenigstens in der Hälfte der ETRD-Ebenen, die in der geometrischen Akzeptanz der Spur liegen, ein Cluster nachgewiesen wird. Der Schnitt auf den Impuls dient dazu, die Zahl der Pionen, die ein ähnliches Signal im ETRD hervorrufen, wie die Elektronen, zu verringern (vgl. Abb. 3.10).

Insgesamt werden im Strip 1 ca. $90 \%$ der Rohdaten verworfen.

Strip 2: In der zweiten Stufe der Datenreduktion, dem Strip 2, werden die Ereignisse auf bestimmte Eigenschaften des Vertex hin getestet. Hierbei wird geprüft

\footnotetext{
${ }^{30}$ von track segments
} 
- ob wenigstens ein Zwei-Prong-Vertex gefunden wurde,

- und wenigstens einer von diesen ein $\chi^{2}$ des Vertexfits von kleiner als 20 aufweist,

- ob in wenigstens einem dieser Vertizes ein Strahlteilchen zugeordnet ist,

- und ob wenigstens einem ein Elektron hinzugeordnet wird,

- ob die Streuwinkel innerhalb der erlaubten Bereiche liegen $\left(0.1<\vartheta_{e}<10.0\right.$ [mrad] und $\left.0.09<\vartheta_{\pi}<100[\mathrm{mrad}]\right)$

Der Strip 2 verwirft ca. $93 \%$ der Ereignisse aus dem ersten Datenreduktionsschritt. Tabelle 5.2 gibt einen Überblick auf die Auswirkungen der einzelnen Schnitte aus den ersten beiden Stufen der Datenreduktion.

\begin{tabular}{|r|l|r|r|r|}
\hline & Schnitt & $\begin{array}{r}\text { Verworfene } \\
\text { Ereignisse }\end{array}$ & $\begin{array}{r}\text { Akzeptierte } \\
\text { Ereignisse }\end{array}$ & Prozent \\
\hline \hline & Strip 1 Run 10000-10848 & & 21179585 & $100 \%$ \\
\hline 1 & wenigstens ein M2-Segment & 312143 & 20867442 & $98.5 \%$ \\
2 & wenigstens ein Elektron & 6411322 & 14456120 & $68.3 \%$ \\
3 & wenigstens ein M2-Segment mit & 5863947 & 8592173 & $40.6 \%$ \\
& zugeordnetem Vertexsegment & & & \\
4 & wenigstens ein Elektron mit einem & 7002503 & 1589670 & $7.5 \%$ \\
& Impuls von weniger als 500 GeV/c & & & \\
\hline \hline & Strip 2 Run 10 000-10848 & & 1589670 & $100 \%$ \\
\hline 5 & wenigstens ein Zwei-Prong-Vertex & 1126385 & 463285 & $29.1 \%$ \\
6 & $\chi_{\text {Vertex }}^{2}<20$ & 289618 & 173667 & $10.9 \%$ \\
7 & wenigstens ein Strahlteilchen & 11979 & 161688 & $10.2 \%$ \\
8 & wenigstens ein Elektron im Vertex & 6700 & 154988 & $9.7 \%$ \\
9 & Streuwinkel in der richtigen & 40814 & 114174 & $7.1 \%$ \\
& Größenordnung & & & \\
\hline \hline & \multicolumn{2}{|c|}{} & 114174 & \\
\hline
\end{tabular}

Tabelle 5.2: Statistik der Datenreduktionsstufen Strip 1 und 2

Strip3 Die 114174 Ereignisse, die nach der Datenreduktion mit dem Strip 1 und 2 als Kandidaten für elastische Streuereignisse gelten, stellen die Grundlage der weiteren Analyse dar. Diese wird in den folgenden Abschnitten ausführlich behandelt.

\subsection{Datenanalyse}

Die Datenanalyse der Strip 2 Daten beginnt wie der Strip 1 mit der Analyse der Elektroninformation, wobei ein besonderes Augenmerk auf der Trennung der ETRDAntwort der auslaufenden Teilchen liegt, bei der zwischen dem gestreuten Elektron und Pion unterschieden werden muß. In einem zweiten Schritt wird daraufhin der Vertex der Wechselwirkung gesucht und geprüft, ob dieser sowohl über ein auslaufendes Elektron als auch über ein einlaufendes Strahlteilchen verfügt. 


\subsubsection{Teilchenidentifikation}

Bei der zu untersuchenden elastischen Streuung gilt es Pionen und Elektronen zu identifizieren. Hierzu werden die Informationen des BTRD und des ETRD herangezogen (vgl. Abschn. 3.3).

Die Teilchenidentifikation des Pions beruht einerseits auf den Informationen des BTRD, welches eine effektive Trennung zwischen Pionen und anderen Strahlteilchen erlaubt. Hierbei werden Strahlteilchen mit mindestens 7 aktiven BTRD-Ebenen als Mesonen identifiziert (vgl. Abschn. 3.3 und Abb. 3.9). Damit wird gewährleistet, dass es sich bei dem einfliegenden Teilchen um ein Pion handelt. Die Identität des ausfliegenden Pions wird nach dem Ausschlußprinzip ermittelt, indem man das Elektron identifiziert.
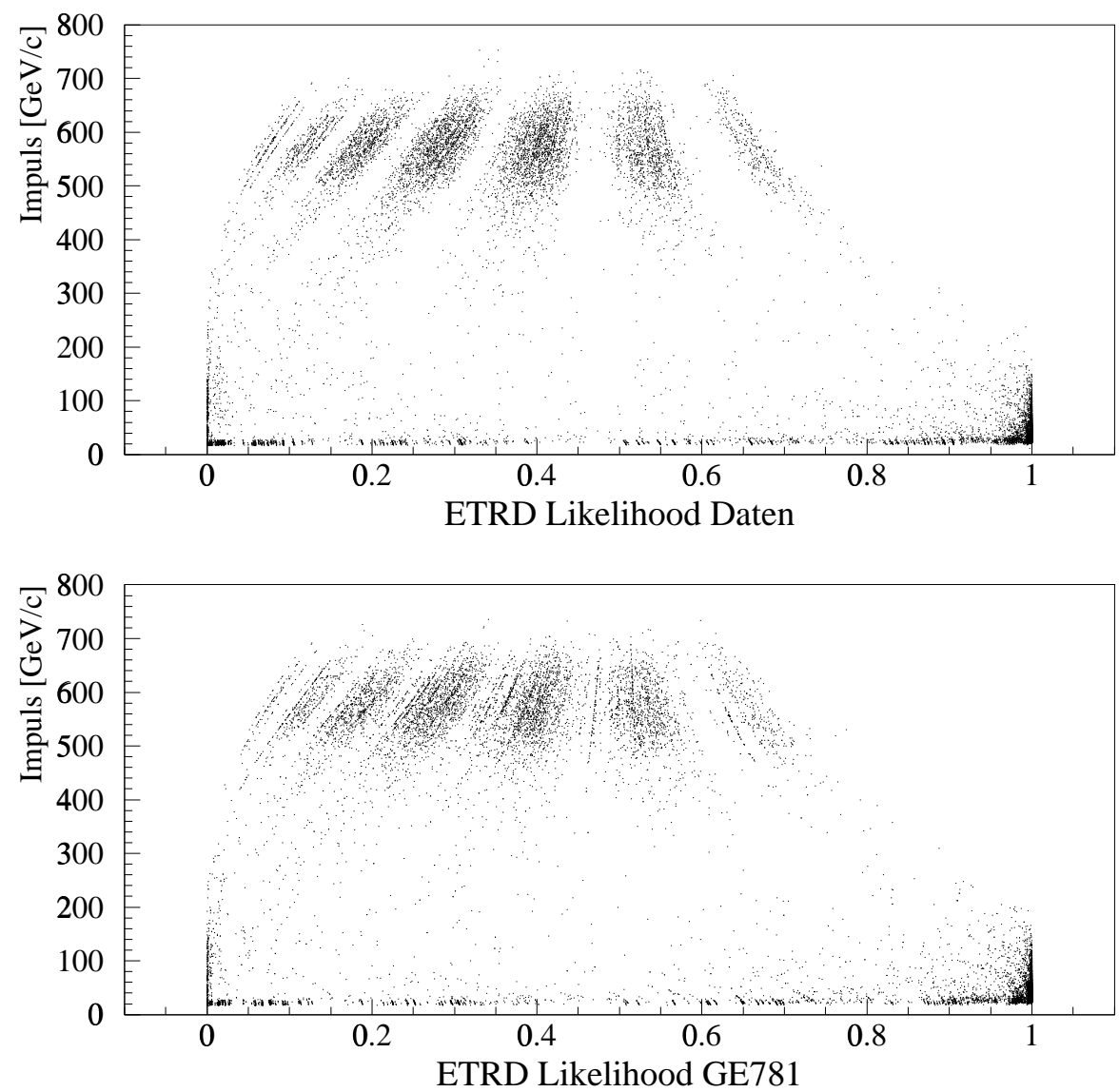

Abbildung 5.1: Impuls gegen die normalisierte Likelihoodfunktion der Elektronen $L_{e l}=\frac{\mathcal{L}_{\pi}}{\mathcal{L}_{e l}+\mathcal{L}_{\pi}}$, wie sie zur Sortierung der Elektronkandidaten verwendet wird. Dabei werden Elektronkandidaten mit einem Impuls von über $350 \mathrm{GeV} / c$ verworfen, da aus dem Wechselwirkungsquerschnitt und aufgrund der Triggerbedingungen keine elastischen Streuereignisse mit Elektronimpulsen dieser Größenordnung erwartet werden können.

Um das Elektron zu identifizieren, muß es unter anderem von der Signatur des Pions im ETRD unterschieden werden, was sich aufwendiger gestaltet, da die Pionen ein den Elektronen ähnliches Signal im ETRD hervorrufen können (vgl. Abb. 3.10). 
Folgende Strategie wird deswegen verwendet.

Da aufgrund des kleinen Wechselwirkungsquerschnitts und des Triggers praktisch keine Ereignisse mit einem $\mathrm{Q}^{2}$ von über $0.35 \mathrm{GeV}^{2} / c^{2}$ erwartet werden und folglich keine Elektronen aus elastischen Streuereignissen mit mehr als gut $350 \mathrm{GeV} / c$ stammen können, werden alle möglichen Elektronkandidaten mit einem Impuls von mehr als $350 \mathrm{GeV} / c$ verworfen, um so eine Kontamination der Daten durch pionischen Untergrund entgegen zu wirken.

Daraufhin wird für alle Spuren im M2-Spektrometer zunächst die Zahl der Cluster entlang der Spur und die Zahl der ETRD-Ebenen, die in der geometrischen Akzeptanz der Spur liegen, bestimmt. Falls mindestens zwei Ebenen innerhalb der Akzeptanz liegen und die Zahl der Cluster pro Ebene bei einem Wert von über 0.5 liegt, dann wird die Spur als möglicher Elektronkandidat weiter untersucht.

Die so gefundenen Elektronkandidaten werden nach ihrer Ladung und der normalisierten Likelihoodfunktion für Elektronen

$$
L_{e l}=\frac{\mathcal{L}_{\pi}}{\mathcal{L}_{e l}+\mathcal{L}_{\pi}}
$$

mit den ETRD-Wahrscheinlichkeiten $\mathcal{L}_{e l}$ und $\mathcal{L}_{p i}$ sortiert, wobei die ETRD-Wahrscheinlichkeiten die Wahrscheinlichkeit angeben, mit der ein Elektron, bzw. Pion, mit dem gemessenen Impuls der zugehörigen Spur die gefundene Zahl an Clustern erzeugt. Abbildung 5.1 zeigt diese normalisierte Likelihoodfunktion für Elektronen für einen Teil der Strip-2-Daten [37]. Falls eine der ETRD-Wahrscheinlichkeiten $\mathcal{L}_{e l}$ oder $\mathcal{L}_{p i}$ nicht berechnet werden kann, stehen als weitere Sortierungsmerkmale die Zahl der Cluster pro Ebene innerhalb der Akzeptanz der Spur, sowie der Impuls zur Verfügung.

Aus der so erhaltenen Liste an Elektronkandidaten werden alle Spuren mit positiver Ladung und solche, die kein Vertex-Segment aufweisen, gestrichen. Bleiben nach dem Streichen dieser Ereignisse noch mehrere Kandidaten übrig, so werden diese mit den Vertex-Informationen abgeglichen.

\subsubsection{Vertex}

Nachdem im Strip 2 schon alle Ereignisse verworfen wurden, die über keinen ZweiProng-Vertex verfügen, werden die Vertex-Kriterien im Strip 3 nochmals genauer untersucht. Hierzu wird dem Vertex mit dem kleinsten $\chi^{2}$ diejenige Spur im Strahlspektrometer mit dem kleinsten räumlichen Abstand zum Wechselwirkungspunkt zugeordnet und geprüft, ob exakt eine Spur aus dem Strahlspektrometer und wenigstens einer der Elektronkandidaten diesem Vertex zugeordnet wird. Weiterhin wird der Schnitt auf das $\chi^{2}$ des Vertexfits von vormals 20.0 in Strip 2 auf 5.0 verschärft. Abbildung 5.2 zeigt die $z$-Position der gefundenen Vertizes für die Daten, wie sie aus dem Strip 2 stammen und für die Daten, die in der Radiusmessung verwendet werden. Für die Analyse werden nur Ereignisse verwendet, die ihren Vertex innerhalb der fünf Targets haben, sprich deren Vertex- $z$-Position zwischen -7 und $+1 \mathrm{~cm}$ liegt. Die Ereignisse deren Vertex strahlaufwärts der Targets liegen, vornehmlich innerhalb der beiden Szintillatoren des Wechselwirkungszählers werden verworfen, da dieser aktiv im Trigger enthalten sind. Die Ereignisse deren Vertex strahlabwärts der Targets liegt, vornehmlich im S4-Szintillator, werden ebenfalls verworfen. 

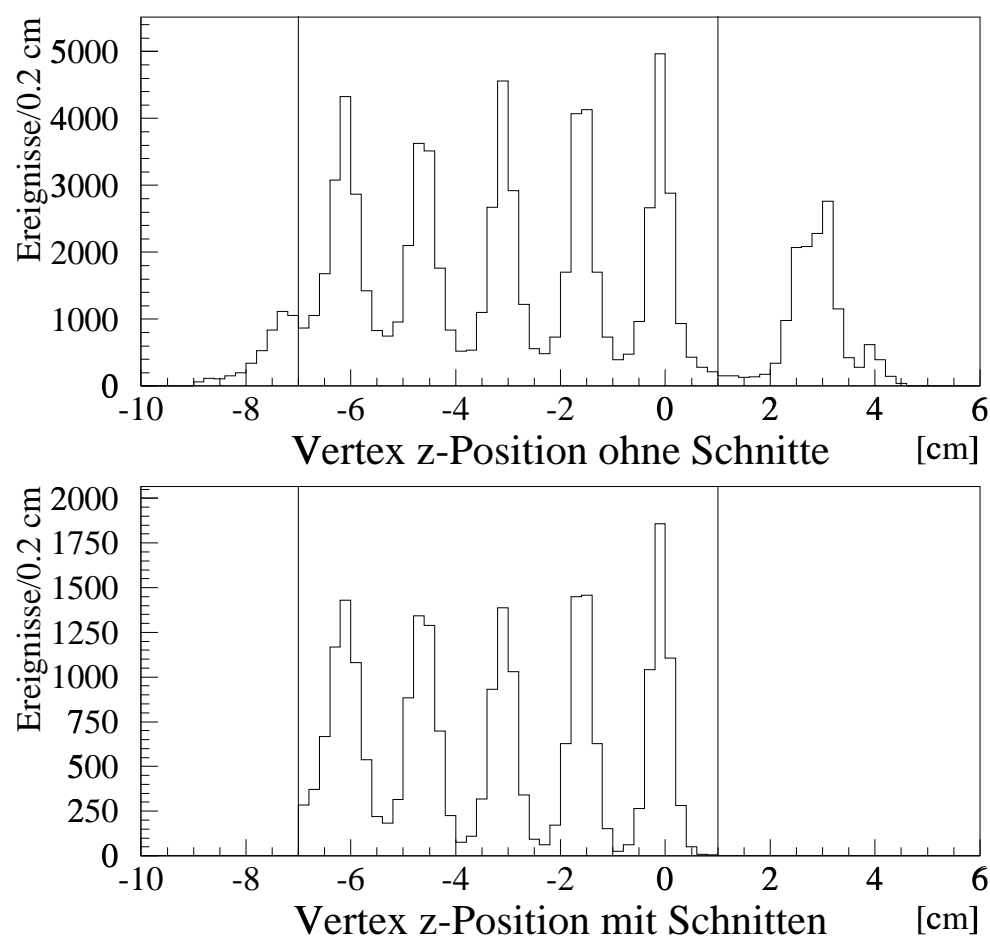

Abbildung 5.2: Vertex z-Positionen ohne Schnitte an die Daten (oben) und mit den Schnitten aus Abschn. 5.2.4 an die Daten (unten). Deutlich zu sehen sind die fünf Targets, sowie als weitere Spitzen strahlaufwärts die Szintillatoren des Wechselwirkungszähler und strahlabwärts der S4-Zähler. In beiden Abbildungen sind die Schnitte auf die $z$-Position des Vertex eingezeichnet.

Für die so erhaltenen Ereignisse werden nun aus den Spurinformationen verschiedene Observablen und ihre Fehler neu errechnet, wie die Streuwinkel, die Koplanarität, aber auch Größen wie die Fehler der Spuren. Weiter wird aus dem Impuls des einlaufenden Pions und dem Elektronstreuwinkel ein Vorhersagewert für den Viererimpulsübertrag berechnet.

\subsubsection{Der Constrained Non-Linear Least Square Fit}

Die elastische Streuung wird durch zwei Parameter vollständig beschrieben, was bedeutet, dass, bei letztlich vier im Fit vorwendeten Observablen, das Problem der $\mathrm{Q}^{2}$ Messung überbestimmt ist.

Ziel eines Non-Linear Least Square Fit ist zu $m$ Observablen $y_{i}$ und den Gewichten $\sigma_{i}$ ein Minimum der Bildfunktion $\chi_{F}(a)$

$$
\chi_{F}(a)=\frac{1}{2} \sum_{i=1}^{m}\left[\frac{y_{i}-f\left(x_{i}, a\right)}{\sigma_{i}}\right]
$$

mit $n$ unbekannten Parametern $a=\left(a_{1}, \ldots, a_{n}\right)^{T} \in \mathrm{R}^{n} \mathrm{zu}$ finden. Die Funktionen $f_{i}(a)$ verknüpfen die Parameter $a$ mit den Observablen $y_{i}$.

Im vorliegenden Fall wird die Fitroutine DSUMSQ der CernLib genutzt. In ihre werden als Parameter der elastischen Streuung der Viererimpuls des einfliegenden 
Pions $p_{\pi}$ und der Viererimpulsübertrag $\mathrm{Q}^{2}$ genutzt. Als Observablen werden der Viererimpuls des einlaufenden Pions $p_{\pi}$, der Viererimpuls des auslaufenden Pions $p_{\pi}^{\prime}$, der Elektronstreuwinkel $\vartheta_{e}$ und der Pionstreuwinkel $\vartheta_{\pi}$ übergeben. Die drei nicht-trivialen Funktionen der vier $f_{i}\left(p_{\pi}, \mathrm{Q}^{2}\right)$ sind durch die Formeln 2.9, 2.7, 2.6 gegeben. Weiterhin wird der Fitroutine die Jakobi-Matrix der Funktion $f(a)$ übergeben.

Als weitere Eingabegrößen für den Non-Linear Least Square Fit werden dann noch die Gewichte der einzelnen Observablen benötigt.

\subsubsection{Selektion elastischer Streuereignisse}

Die Selektion elastischer Streuereignisse erfolgt in zwei Stufen. Zum einen werden die Rohdaten wie in den vorangegangenen Abschnitten erläutert aufgrund von topologischen Eigenschaften der elastischen Streuung, wie der Zahl der auslaufenden Spuren, einer Elektronspur im M2-Spektrometer mit hinzugefügtem Vertex-Segment usw., sortiert. Zum anderen können die Eigenschaften der so erhaltenen Ereignisse im NonLinear Least Square Fit auf ein elastisches Verhalten hin getestet werden.

Die zentrale Größe beim diesem Fit ist das $\chi_{F}^{2}$, also die gewichtete Summe der Abweichungen der Fitvorhersagewerte von den Meßwerten, welche es für den Fit zu minimieren gilt. Bei elastische Ereignisse sollten die Observablen innerhalb der Auflösung mit den realen physikalischen Gegebenheiten übereinstimmen und somit im Fit, aufgrund der Eindeutigkeit der dem Fit zugrundeliegenden Relationen, zu $95 \%$ ein $\chi_{F}^{2}$ von kleiner als drei ergeben. Die Anteile der einzelnen Observablen $y_{i}$ am $\chi_{F}^{2}$ läßt sich hierbei über die Pulls

$$
y_{\text {pull }}=\frac{y_{\text {meas. }}-y_{f i t}}{\sigma_{m e a s}}
$$

der einzelnen Observablen bestimmen und steht somit als Maß für die Elastizität neben den primären Observablen zur Verfügung. Primäre Observablen sind in diesem Zusammenhang die drei Impulse des einlaufenden und der zwei auslaufenden Teilchen und die zwei Streuwinkel, errechnet aus den Impulsrichtungen der drei Teilchen. Aus diesen primären Observablen lassen sich Größen wie die Pulls, die Elastizität oder die Koplanarität berechnen.

Unter der Koplanarität versteht man das Spatprodukt der drei Richtungsvektoren der Spuren, der an der Streuung beteiligten Teilchen

$$
\operatorname{copl}=\frac{\vec{p}_{\pi}^{\prime} \times \vec{p}_{e}^{\prime}}{\left|\vec{p}_{\pi}^{\prime} \times \vec{p}_{e}^{\prime}\right|} \cdot \frac{\vec{p}_{\pi}}{\left|\vec{p}_{\pi}\right|}
$$

Da bei der elastischen Streuung die drei Spuren in einer Ebene liegen, bietet sich die Koplanarität als Kontrollgröße an. Da aufgrund von Auflösungseffekten eine von Null verschiedene Koplanarität zu erwarten ist, die zusätzlich für kleinere $\mathrm{Q}^{2}$ eine größere Varianz aufweist, liegt es nahe statt der Koplanarität selbst das $\chi^{2}$ der Koplanarität zu betrachten.

Die Elastizität ist definiert als die Differenz der Impulse des einlaufenden Teilchens und der auslaufenden Teilchen

$$
\text { elast }=p_{\pi}-p_{\pi}^{\prime}-p_{e}^{\prime}
$$



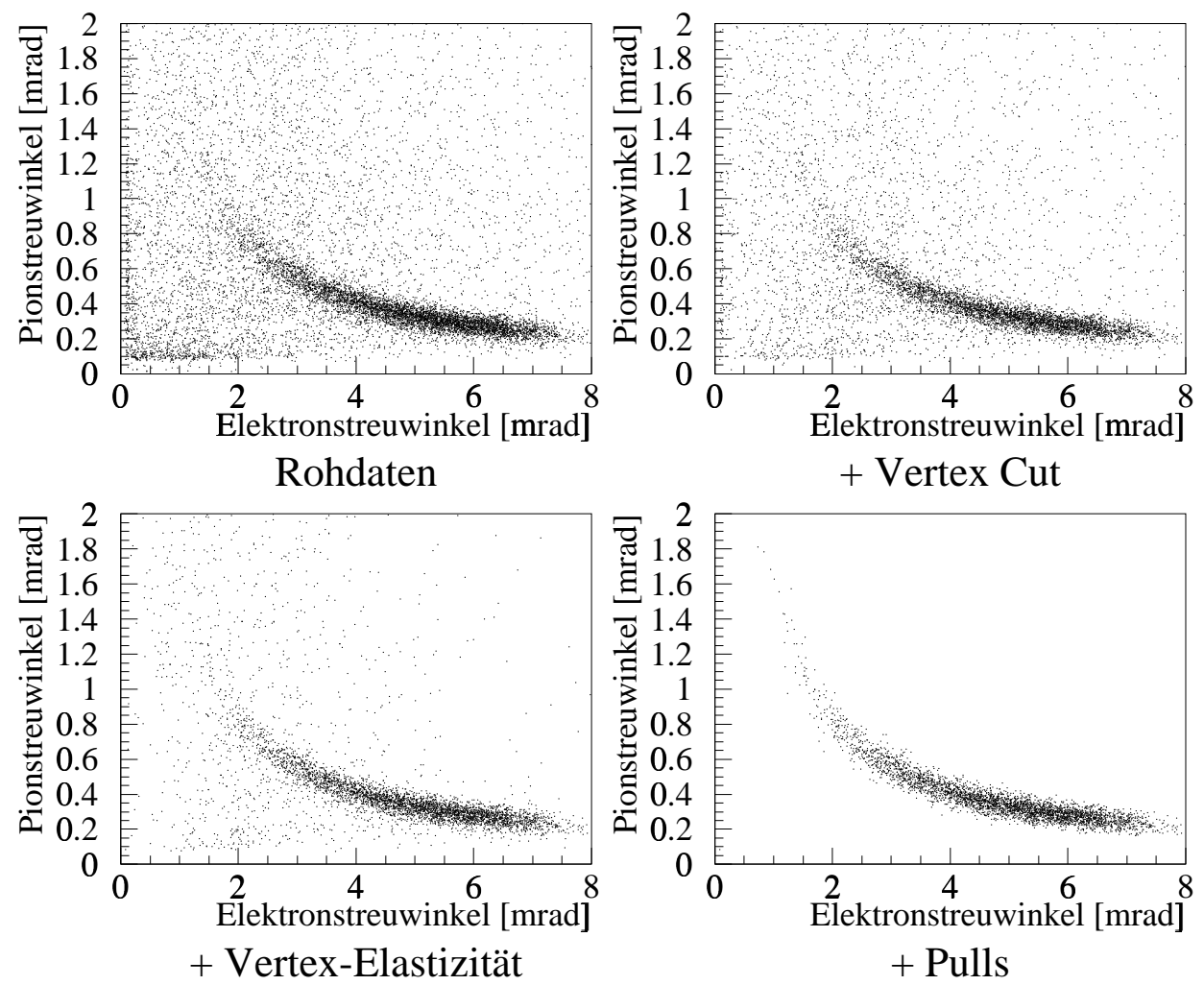

Abbildung 5.3: Winkel-Winkel-Korrelation für verschiedene Schnitte. Oben links die „Rohdaten“ aus dem Strip 2, für die ein Fit des Viererimpulsübertrags möglich ist, da Vertex und Elektron gefunden werden. Oben rechts diese Daten mit einem Schnitt von $\chi^{2}$ des Vertexfits kleiner fünf. In der unteren Reihe von links nach rechts mit einer Vertex-Elastizität kleiner $30 \mathrm{GeV} / c$ und mit den Schnitten auf den Elektron-Pull kleiner 3.0 und auf den Pion-Pull kleiner 3.5.

Da der Elektronimpuls durch Bremsstrahlungsprozesse stark verfälscht wird, wird statt der Elastizität die sog. Vertex-Elastizität betrachtet

$$
\text { Vertex }- \text { Elastizität }=p_{\pi}-p_{\pi}^{\prime}-\mathrm{Q}_{f i t}^{2} / 2 m_{e},
$$

bei der Elektronimpuls aus dem $\mathrm{Q}^{2}$ des Fits berechnet wird, um so den Einfluß der Bremsstrahlung des Elektrons zu unterdrücken.

Die elastischen Ereignisse werden in der Analyse über Schnitte auf die Pulls des Elektron- und Pionstreuwinkels, der Vertex-Elastizität und auf das $\chi^{2}$ des Vertexfits selektiert. Die Pulls und die Vertex-Elastizität sind in Abb. 5.4 für die Rohdaten aus Strip 2 und für die Ereignisse, welche im Non-Linear Least Square Fit verwendet werden, gezeigt. Zusätzlich wird deren Verhalten, wie es sich aus GEANT-Simulationen ergibt, gezeigt. Abbildung 5.3 zeigt das Verhalten der Winkel-Winkel-Korrelation der Daten unter verschiedenen Schnitten (vgl. auch Abb. 2.3). Die Breite der WinkelWinkel-Korrelation wird einerseits durch die Strahlimpulsverteilung mit einer Breite von ca. $60 \mathrm{GeV} / c$ und andererseits durch die Auflösungsverschmierung hervorgerufen.

Tabelle 5.3 gibt Auskunft über die Zahl der Ereignisse, die in den einzelnen Schritten der Analyse verworfen werden. Letztlich bleibe von den 36 Millionen aufgezeichneten Ereignissen der Runperiode 10000 bis 11000 nach der Analyse 24118 Ereignisse übrig. 
Rohdaten
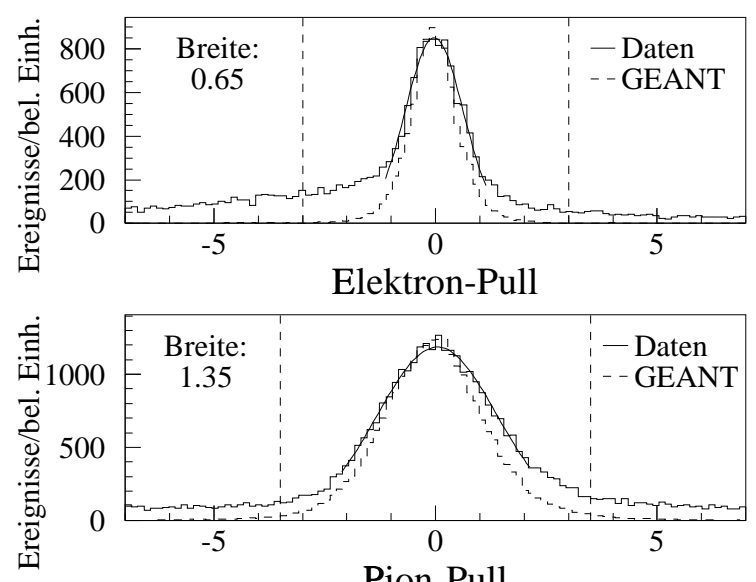

Pion-Pull

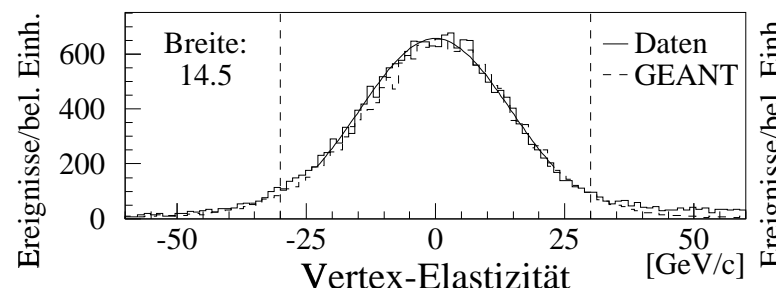

Daten im Radiusfit
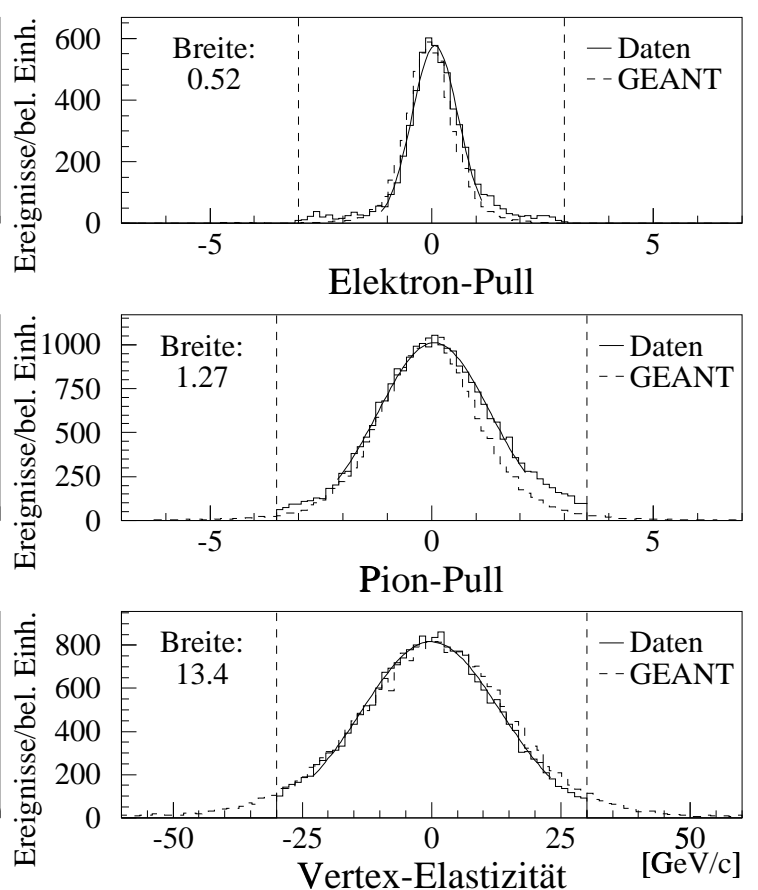

Abbildung 5.4: Pull des Elektronwinkels und des Pionwinkels sowie die VertexElastizität für die Daten aus dem Strip 2 (linke Spalte) und die im Radiusfit verwendeten Ereignisse (rechte Spalte).

\begin{tabular}{|c|c|c|c|c|}
\hline & Schnitt & $\begin{array}{r}\text { Verworfene } \\
\text { Ereignisse }\end{array}$ & $\begin{array}{r}\text { Akzeptierte } \\
\text { Ereignisse }\end{array}$ & Prozent \\
\hline & Strip 3 Run $10000-10848$ & & 114174 & $100 \%$ \\
\hline 1 & wenigstens ein Elektronkandidat & 4653 & 109521 & $95.9 \%$ \\
\hline 2 & $\begin{array}{l}\text { wenigstens ein Elektronkandidat } \\
\text { mit negativer Ladung }\end{array}$ & 214 & 109307 & $95.7 \%$ \\
\hline 3 & $\begin{array}{l}\text { wenigstens ein Elektronkandidat } \\
\text { mit zugeordnetem Vertexsegment }\end{array}$ & 6829 & 102478 & $89.8 \%$ \\
\hline 4 & $\begin{array}{l}\text { wenigstens ein Elektron mit einem } \\
\text { Impuls von weniger als } 350 \mathrm{GeV} / c\end{array}$ & 3058 & 99420 & $87.1 \%$ \\
\hline 5 & $\begin{array}{l}\text { wenigstens ein Vertex mit einem } \\
\chi^{2} \text { kleiner } 5.0\end{array}$ & 12008 & 87412 & $76.6 \%$ \\
\hline 6 & $\begin{array}{l}\text { genau ein dem Vertex zugeordnetes } \\
\text { Strahlteilchen }\end{array}$ & 13011 & 74401 & $65.2 \%$ \\
\hline 7 & $\begin{array}{l}\text { wenigstens ein dem Vertex } \\
\text { zugeordneter Elektronkandidat }\end{array}$ & 471 & 73930 & $64.8 \%$ \\
\hline 8 & Zwei-Prong-Berechnungen & 2836 & 71094 & $62.3 \%$ \\
\hline \multirow[t]{2}{*}{9} & Analyseschnitte zur Ereignisauswahl & 46976 & 24118 & $21.1 \%$ \\
\hline & & & 24118 & \\
\hline
\end{tabular}

Tabelle 5.3: Statistik der Datenanalyse 


\subsubsection{Auflösung}

Die Auflösung einzelner Observabeln läßt sich aus simulierten Ereignissen über den Vergleich des von SOAP rekonstruierten Wertes mit dem von GEANT hineingegeben bestimmen. Die so erhaltenen Auflösungen gehen als die Gewichte der einzelnen Observablen in den Non-Linear Least Square Fit ein.

Die Auflösung der Observablen hängt zu einem von den einzelnen Detektorauflösungen ab, welche von der Zahl der getroffenen Drähte, bzw. der getroffenen Mikrostreifen, und von deren Abstand beeinflußt wird. Hierbei spielt es für die Orts- und Impulsauflösung der hochenergetischen Spuren vorallen ein Rolle, ob die LASDs getroffen wurden oder nicht.

Die Streuwinkelauflösungen werden aus den Spursegmente im Vertex-Spektrometer berechnet und deren Auflösung wird durch drei Effekte reduziert. Zum einen reduzieren Vielfachstreuungen zu Auflösung, wobei diese mit dem zu durchquerenden Material bis zu dem Detektor skaliert.
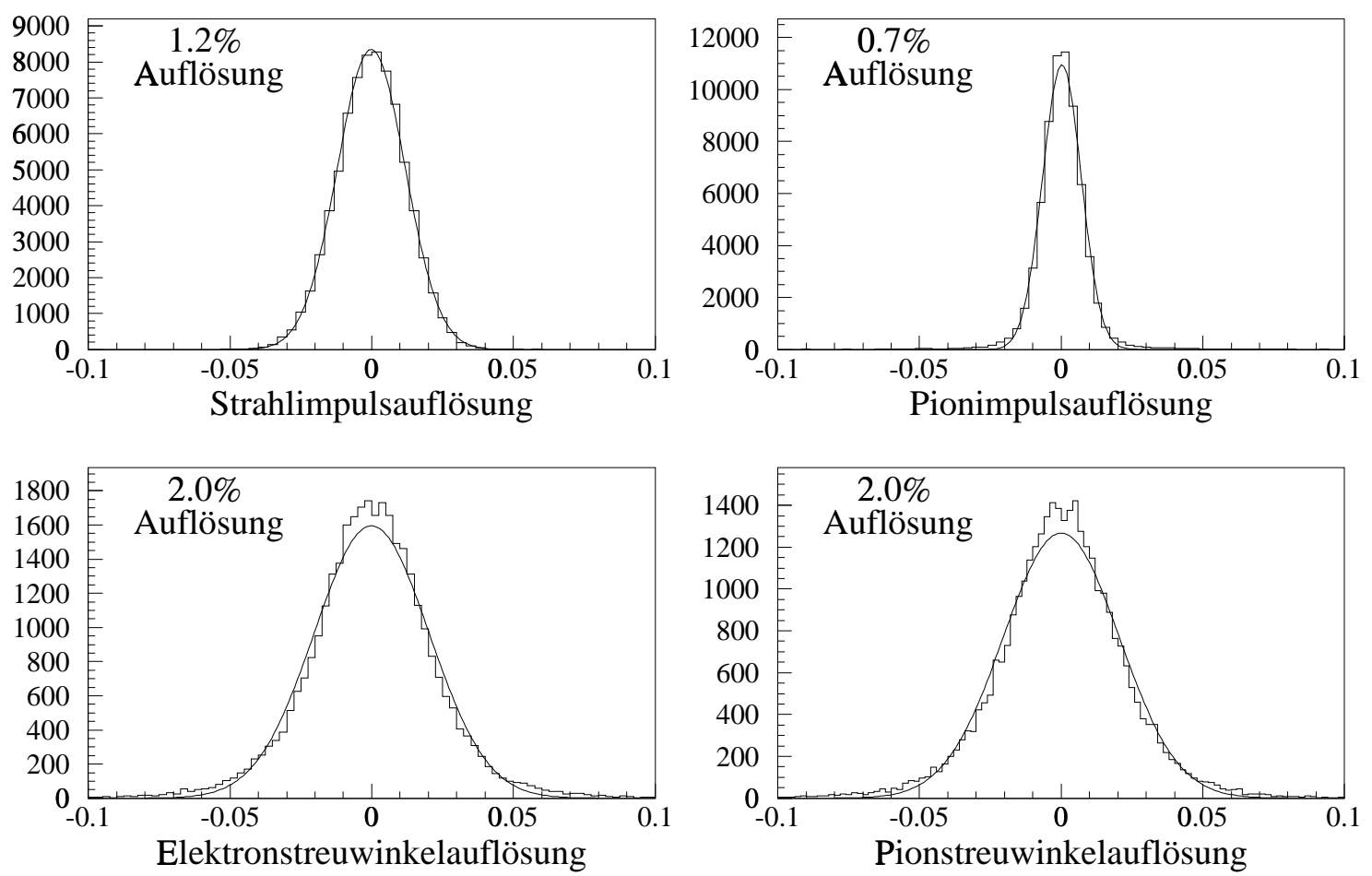

Abbildung 5.5: Auflösungen der im Constrained Non-Linear Least Square Fit verwendeten Observablen, wobei es sich für die Streuwinkelauflösungen um mittlere Auflösungen handelt.

Auf den Weg durch Materie erfährt ein geladenes Teilchen eine Vielzahl von kleinen Ablenkungen, wobei die meisten hiervon auf Coulomb Streuung an Kernen zurück zuführen sind. Die mittlere Ablenkung durch diese vielfach Streuung $\left(\mathrm{MCS}^{31}\right)$ folgt für kleine Ablenkungen in etwa einer Gaußschen Verteilung (Näherung der Moliere

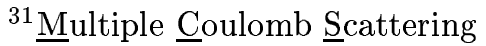


Formel), für dessen Breite $\theta_{0}$ die Relation

$$
\theta_{0}=\frac{13.6 \mathrm{MeV}}{\beta c p} z \sqrt{x / X_{0}}\left[1+0.018 \ln \left(x / X_{0}\right)\right]
$$

gilt. Hierbei sind $p, \beta c$ und $z$ der Impuls, die Geschwindigkeit und die Ladung des einlaufenden Teilchens, und $x / X_{0}$ ist die Dicke des Materials in Strahlungslängen.

Der Effekt der MCS ist für die mit dem Elektron verknüpften Observablen von besonderer Bedeutung, da die Elektronimpulse typischerweise in der Größenordnung von $25-300 \mathrm{GeV} / c$ liegen. Weiterhin wird die Auflösung von der Zahl der Targets, die von den auslaufenden Teilchen durchquert werden müssen, beeinflußt (vgl. Abb 5.6).
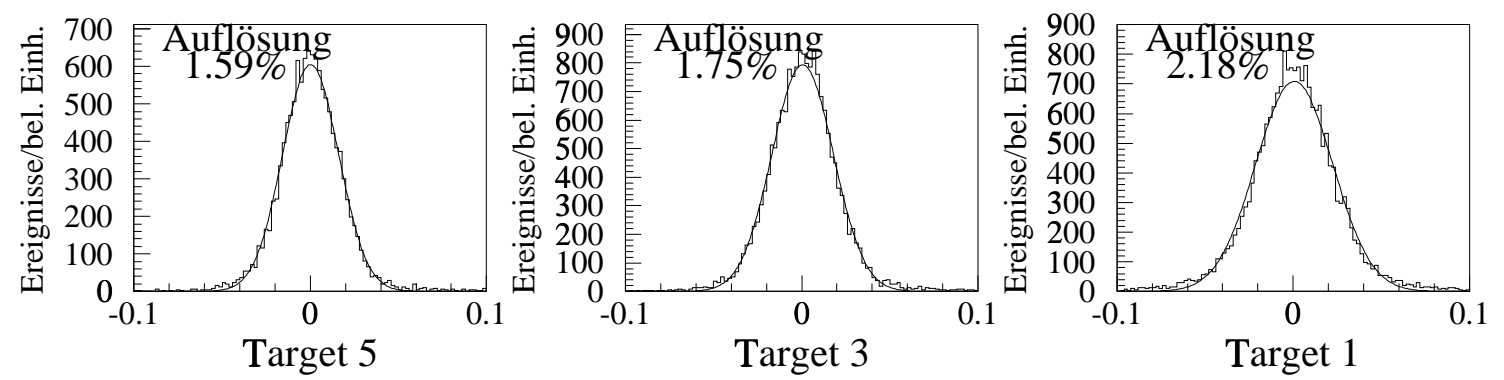

Abbildung 5.6: Abhängigkeit der Auflösung des Elektronstreuwinkels. In der oberen Reihe ist die Auflösung des Elektronstreuwinkels für verschiedene Targets gezeigt. Beim Target 5 (links) muß das Elektron nur noch den Wechselwirkungszähler durchqueren, bevor es im Vertex-Spektrometer vermessen wird. Beim Target 1 (rechts) durchquert es zusätzlich die vier anderen Target, was den Effekt der MCS vergrößert. Entsprechend liegt die Auflösung des Elektronstreuwinkels beim Target 3 (Mitte) zwischen den Werten von Target 1 und 5 .

Weitere die Streuwinkelauflösung betreffende Effekte sind die Bremsstrahlung des Elektrons, welche wie die Vielfachstreuung mit dem durchlaufenden Material skaliert, und die Auflösung der Spurrekonstruktion.

\subsection{Der Radiusfit}

Der elektromagnetischen Ladungsradius der Pionen wird aus den Daten gewonnen, indem man die $\mathrm{Q}^{2}$-Verteilung durch den Mott-Wirkungsquerschnitt teilt und an den so erhaltenen Formfaktor einen Monopolfit (Gl. 2.34) ansetzt (vgl. Abschn. 2.2 und Abb. 2.5). Hierbei muß der aus den Daten gewonnene Formfaktor mit den Korrekturen aus der Akzeptanz des Detektors und des Triggers (vgl. Abschn. 4.2), sowie mit den Strahlungskorrekturen (vgl. Abschn. 2.3) versehen werden.

Abbildung 5.7 zeigt alle Stufen vom $\mathrm{Q}^{2}$-Spektrum bis zum Radiusfit. Oben links das $\mathrm{Q}^{2}$-Spektrum der Daten mit den in der Legende aufgeführten Schnitten und der sich aus diesen ergebende Formfaktor (oben rechts). In der mittleren Reihe die Akzeptanz des Detektors (vgl. Abb. 4.8) und die gesamtkorrektur inklusive Trigger (vgl. Abb. 4.7) und Strahlungskorrekturen (vgl. Abb. 2.7). Unten schließlich der korrigierte Formfaktor mit den Radiusfit im $\mathrm{Q}^{2}$-Bereich von 0.04 bis $0.1725 \mathrm{GeV}^{2} / c^{2}$. 



Formfaktor aus den Daten

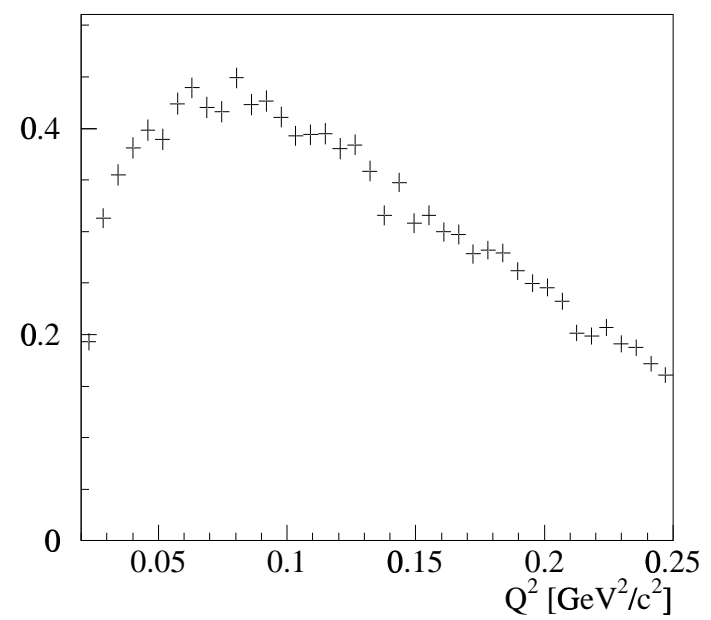

Akzeptanz inc. Trigger

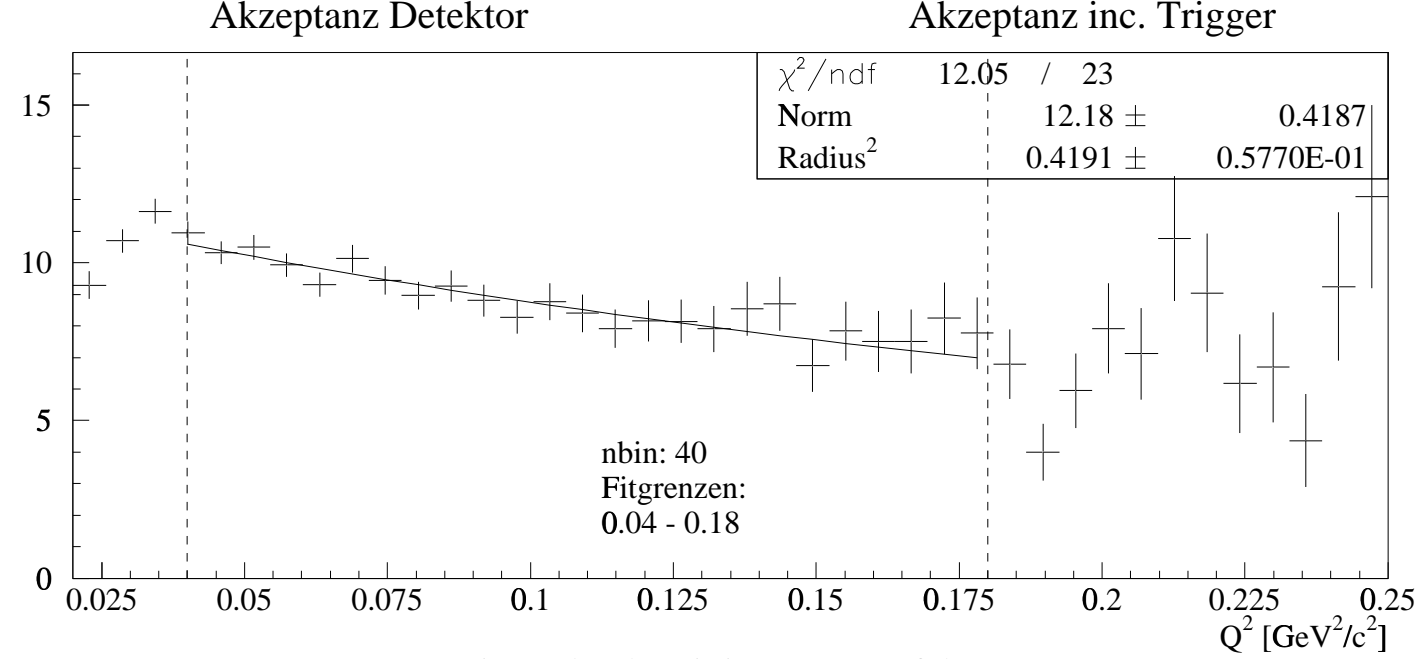

Fit an den korrigierten Formfaktor

Abbildung 5.7: Vom gemessen $\mathrm{Q}^{2}$-Spektrum zum Radiusfit (vgl. Text). 


\subsection{Systematische Studien}

\subsubsection{Abhängigkeiten von den Schnitten}

Die Ereignisauswahl der elastischen Ereignisse aus den Daten des Strip 2 beeinflußt das Ergebnis des Radiusfits. Diese Abhängigkeiten werden untersucht indem einzelne Schnitte variiert werden und das Verhalten des Radiusfits hierzu geprüft wird. Abbildung 5.8 zeigt diese Abhängigkeiten des Radiusfits von den Schnitten. Die markierten Werte von $\chi^{2}$ des Vertexfits gleich 5.0, einem Absolutbetrag der Vertex-Elastizität von weniger als $30 \mathrm{GeV} / c$, einem Absolutbetrag des Elektron-Pulls von kleiner als 3.0 und einem Absolutbetrag des Pion-Pull von kleiner als 3.5 stellen für die einzelnen Abbildungen in Abb. 5.8 die Basis dar, auf deren die einzelnen Schnitte variiert werden. Deutlich zu erkennen ist, dass die beiden Pulls ab einem Wert von ca. drei den Radius praktisch nicht mehr beeinflussen, während der Einfluß des Schnitts auf das $\chi^{2}$ des Vertexfits und die Vertex-Elastizität zu Schwankungen in Ergebnis des Radiusfits führen, ohne dabei ihn dabei in starkem Maße systematisch zu verändern.
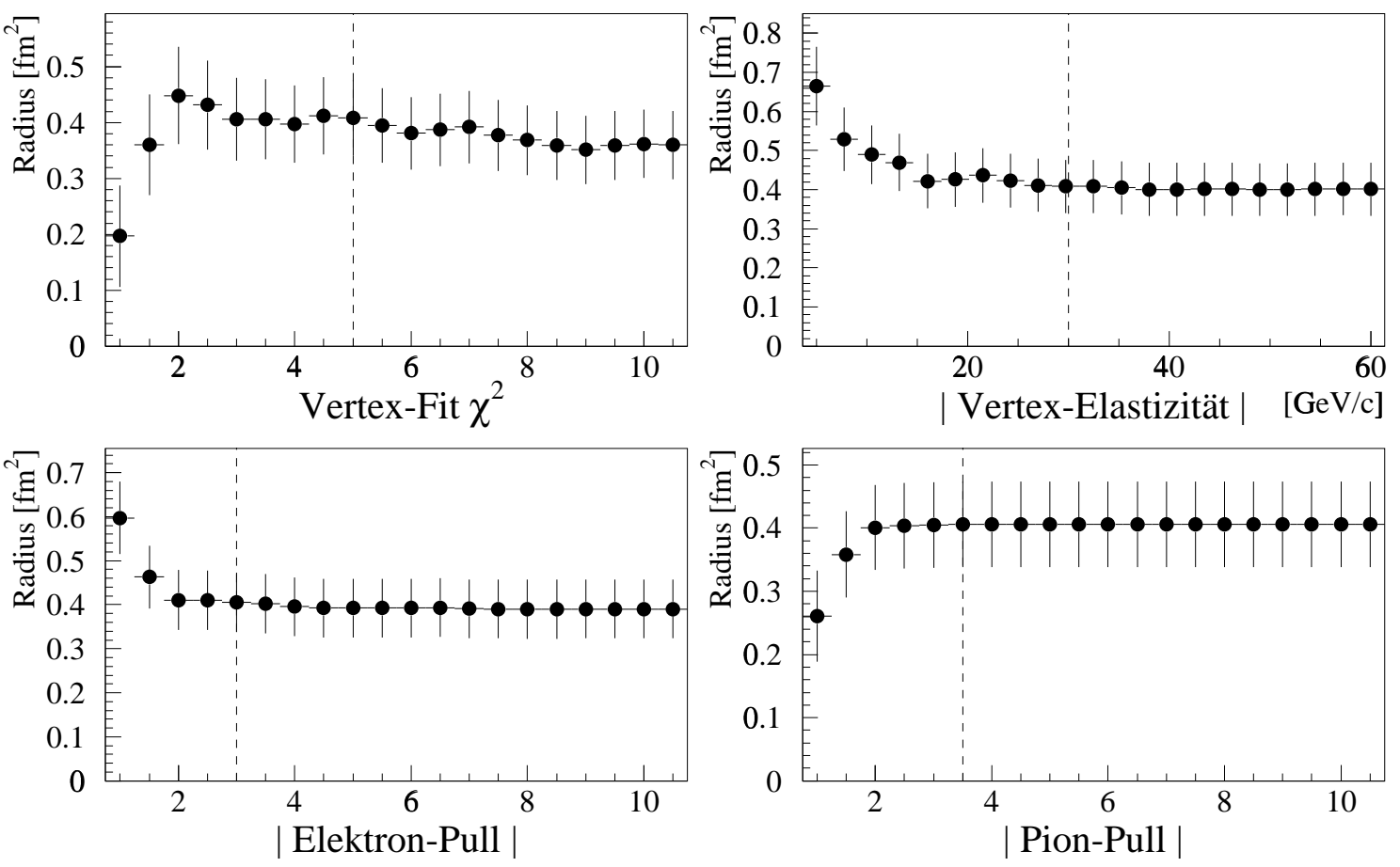

Abbildung 5.8: Abhängigkeit des Radius von den Schnitten. Als Basis dienen die eingezeichneten Schnitte. Dies sind im einzelnen der Schnitt auf das $\chi^{2}$ des Vertexfits kleiner fünf, der Absolutbetrag des Elektron-Pulls kleiner als drei, sowie der Absolutbetrag des Pion-Pull kleiner 3.5 und der Vertex-Elastizität kleiner $30 \mathrm{GeV} / c$.

\subsubsection{Abhängigkeiten von den Fitgrenzen}

Das Ergebnis des Radiusfits ist in zweierlei Hinsicht von den Fitgrenzen abhängig. Zum einen verändert sich der gefittet Radius mit den Grenzen des Fitintervalls und zum anderen verändert sich auch der statistische Fehler, da so mehr oder weniger Ereignisse in den Fit aufgenommen werden. Dieser zweite Effekt hat vorallem bei der 




Radius gegen untere Fitgrenze

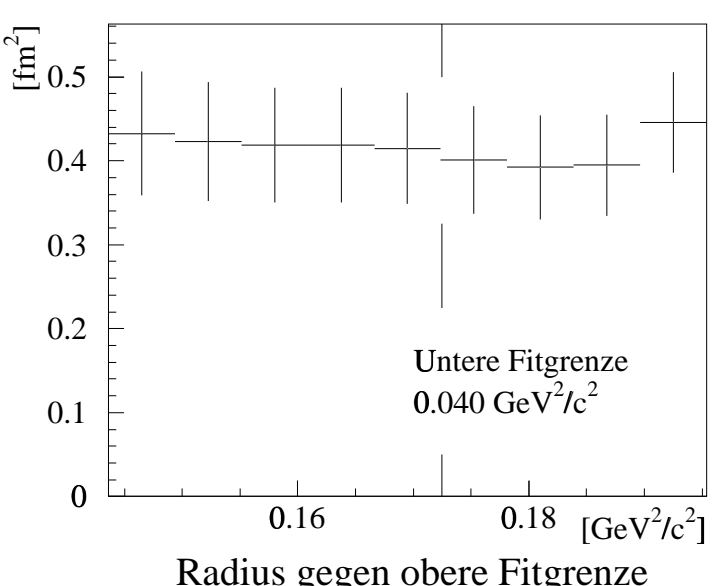

Radius gegen obere Fitgrenze

Abbildung 5.9: Abhängigkeit der Radius von den Fitgrenzen.

unteren Fitgrenze einen großen Einfluß, da die Zahl der Ereignisse bei kleinem $\mathrm{Q}^{2}$ stark ansteigt, bis sie ab ca. $0.03 \mathrm{GeV}^{2} / c^{2}$ aufgrund der hier endenden geometrischen Akzeptanz wieder abfällt (vgl. das $\mathrm{Q}^{2}$-Spektrum aus den Daten in Abb. 5.7). Ein weiterer Effekt, der hieraus resultiert, ist die Tatsache, das die Korrekturen, welche sich aus der Untersuchung der Akzeptanz mit dem GEANT-Paket ergeben, in diesen Bereich der kleinen $\mathrm{Q}^{2}$ stark zunimmt.

Abbildung 5.9 zeigt die Abhängigkeit des gemessenen Radius und der statistischen Fehlers von den Fitgrenzen.

\subsubsection{Statistischer und systematischer Fehler}

Bei der Ermittlung des statistischen und systematischen Fehlers für den gemessenen Radius ergibt sich der statistische direkt aus dem Fit $\mathrm{zu} \pm 0.057 \mathrm{fm}^{2}$. Der systematische Fehler setzt sich aus verschiedenen Quellen zusammen.

- Die Abhängigkeiten von den verschiedenen Analyseschnitten ergibt einen Beitrag zum systematischen Fehler.

- Die GEANT-Simulationen des Detektorverhaltens sind in ihrer Genauigkeit begrenzt und führen zu einem weiteren Beitrag.

- Das Triggerverhalten läßt sich nicht vollständig simulieren und trägt ebenfalls zum systematischen Fehler bei.

- Die Wahl der Fitgrenzen liefert einen Beitrag zum systematischen Fehler.

- Da die Informationen aus den Photon-Kalorimeter nicht in die Analyse eingehen und das GEANT-Paket über keinen Generator für inelastische Ereignisse verfügt, deren Verhalten unter den angewendeten Schnitten untersucht werden könnte, kann der Cut-Off-Parameter der Strahlungskorrekturen nur abgeschätzt werden und führt zu einer weiteren systematischen Unsicherheit. 
- Apparative Ungenauigkeiten, wie Schwankungen im Alignment oder in den Magnetfelder sind bei der Untersuchung systematischen Fehler ebenfalls zu berücksichtigen.

Die einzelnen Beiträge addieren sich quadratisch, solange sie linear unabhängig sind. Allerdings lassen sich nicht alle Beiträge zum systematischen Fehler in linear unabhängige Beiträge zerlegen. So sind die Analyseschnitte auf die Pulls des Elektronund des Pionstreuwinkels nicht unabhängig, wie ein Vergleich der von ihnen verworfenen Ereignisse und deren Einfluß auf den Radius zeigt. Solche Beiträge, die linear von einander abhängen, addieren sich linear.

Die Beiträge der einzelnen Analyseschnitte kann aus Abb. 5.8 abgeschätzt werden. Dabei fallen die Schnitte auf die Pulls mit einem Beitrag von $0.010 \mathrm{fm}^{2} \mathrm{kaum}$ gegenüber den Variationen des Vertex $\chi^{2}$ und der Vertex-Elastizität ins Gewicht. Die Variation des Vertex $\chi^{2}$ um ein $\sigma$ führt hierbei zu einer Radiusveränderung von $0.015 \mathrm{fm}^{2}$. Eine Variation der Vertex-Elastizität in der Größenordnung von ca. $5 \mathrm{GeV} / c$ ergibt ebenfalls eine Variation von $0.015 \mathrm{fm}^{2}$ in dem Radiuswert. All diese Beiträge erweisen sich als linear abhängig von einander und folglich summiert sich ihr gemeinsamer Beitrag zu dem systematischen Fehler zu $0.040 \mathrm{fm}^{2}$.

Um den Einfluß von GEANT-Ungenauigkeiten abzuschätzen, wurden die Korrekturen, die sich aus den mit GEANT errechneten Akzeptanzen ergeben, um einem zusätzlichen Faktor von $5 \%$ angehoben. Die sich dabei ergebende Radiusänderung beträgt $0.02 \mathrm{fm}^{2}$, wobei diese Änderung ihren größten Einfluß im $\mathrm{Q}^{2}$-Bereich von unter $0.06 \mathrm{GeV}^{2} / c^{2}$ hat, wo die Akzeptanz des Detektors deutlich abnimmt. Dieser Effekt ist auch bei der Diskussion des fitgrenzenabhängigen Teils der systematischen Fehler zu berücksichtigen. Die Grundlage der Abschätzung dieses Beitrags der Fitgrenzen zu dem systematischen Fehler liefert Abb. 5.9. Bei der Variation der unteren Fitgrenze ist der Radius bis hinunter zu $0.04 \mathrm{GeV}^{2} / c^{2}$ nahezu konstant und unterhalb dieser Grenze beginnt ein Bereich in dem der Einfluß der Detektor Akzeptanz dominant wird. Der Einfluß der Fitgrenzen auf den Radius beläuft sich insgesamt auf rund $0.02 \mathrm{fm}^{2}$. Den größten Beitrag zum systematischen Fehler liefert das nicht vollständig verstandene Triggerverhalten. Ersetzt man die in dieser Analyse verwendeten Effizienzen der Hodoskop 1 Elemente aus der unabhängigen Analyse des Hodoskops durch jene, die sich aus der Anpassung des $x$-Positionsspektrums ergeben (vgl. Abschn. 4.2.2 und Abb. 4.6), so ergibt sich eine Veränderung des Radius um ca. $0.06 \mathrm{fm}^{2}$. Der Einfluß $\operatorname{der} \mathrm{Q}^{2}$-Auflösung ist ebenso vernachlässigbar, wie etwaige Binningeffekte.

Der systematische Gesamtfehler addiert sich damit quadratisch zu $0.077 \mathrm{fm}^{2}$ (vgl. Tab. 5.4). 


\begin{tabular}{|c|c|}
\hline Fehlerquelle & Größe \\
\hline \multicolumn{2}{|c|}{$\overline{\text { Ereignisauswahl (Schnitte) }}$} \\
\hline Vertex $\chi^{2} \pm 1 \sigma$ & $0.015 \mathrm{fm}^{2}$ \\
\hline Vertex-Elastizität $\pm 5 \mathrm{GeV} / c$ & $0.015 \mathrm{fm}^{2}$ \\
\hline Elektron- und Pion-Pull $\pm 1 \sigma$ & $0.010 \mathrm{fm}^{2}$ \\
\hline Zwischensumme linear & $0.040 \mathrm{fm}^{2}$ \\
\hline Akzeptanzungenauigkeiten & $0.020 \mathrm{fm}^{2}$ \\
\hline Triggerungenauigkeiten & $0.060 \mathrm{fm}^{2}$ \\
\hline Variation der Fitgrenzen & $0.020 \mathrm{fm}^{2}$ \\
\hline " systematischer Gesamtfehler & $0.077 \mathrm{fm}^{2}$ \\
\hline
\end{tabular}

Tabelle 5.4: Auflistung der einzelnen Beiträge zu dem systematischen Fehler. 


\section{Ergebnis und Diskussion}

\subsection{Diskussion der Analyse}

Die Analyse des mittleren quadratischen Ladungsradius der Pionen im Rahmen des SELEX Experiments wird durch drei Effekte in der Qualität des Ergebnisses beschränkt. Zum einen weisen alle mit den Elektron verknüpften Observablen Probleme mit Vielfachstreuungen und Bremsstrahlungsprozessen auf, welche vor allem die Verwendung des Elektronimpulses als Observable verbieten. Dieser Effekt skaliert mit der Menge an Material das hinter den Targets im Strahl steht, was im Rahmen des SELEX Experiments vor allem durch die Silizium-Mikrostreifen-Detektoren im Vertex-Bereich (VSSDs) eingebracht wird. Zum anderen ist der Einfluß der sich aus den GEANTSimulationen ergebenden Akzeptanzkorrekturen auf den Radius groß, da die Akzeptanz des Detektors systematisch zu kleineren $\mathrm{Q}^{2}$ hin abfällt und im $\mathrm{Q}^{2}$-Bereich unterhalb von $0.06 \mathrm{GeV}^{2} / c^{2}$ abknickt (vgl. Abb. 4.9). Die starken $\mathrm{Q}^{2}$-Abhängigkeiten des Triggers übt einen weiteren Einfluß auf das Ergebnis der Radiusmessung aus. Aus der Triggerlogik ergibt sich ab einem $\mathrm{Q}^{2}$ von $0.1 \mathrm{GeV}^{2} / c^{2}$ eine zusätzlicher Abfall in der Akzeptanz des Triggers. Das Triggerverhalten hängt zusätzlich von den Effizienzen der einzelnen Hodoskopelemente ab, welche einen nicht zu vernachlässigen Einfluß auf den Radius ausüben (vgl. Abschn. 4.2.2).

Im Vergleich zu der ersten Analyse der elastischen Streudaten ([38] und [39]) sind Fortschritte im gesamten Verständnis des Detektors erzielt worden. Hierzu trägt vor allem das GEANT-Paket bei, welches Aufschluß über viele Details im Antwortverhalten des Detektors liefert. Der Einfluß des Triggers auf die Messung ist besser verstanden, auch wenn sich die hiermit verbundenen systematischen Probleme nicht lösen lassen.

Im Vergleich zu dem vorläufigen Ergebnis der ersten Analyse [38] von $\left\langle r^{2}\right\rangle=$ $0.45 \pm 0.03$ (stat) \pm 0.07 (syst) $\mathrm{fm}^{2}$, basierend auf einen maximum likelihood fit an den Wirkungsquerschnitt, der sich aus 26000 Ereignissen im $\mathrm{Q}^{2}$-Intervall von 0.026 bis $0.2 \mathrm{GeV}^{2} / c^{2}$ errechnet, sind die Beiträge zum systematischen Fehler besser verstanden. Die in der ersten Analyse verwendete Fitmethode an den von $\mathrm{Q}^{-4}$-Abfall dominierten Wirkungsquerschnitt erlaubt es nicht die im Formfaktorplot offensichtlichen Probleme im $\mathrm{Q}^{2}$-Bereich unterhalb von $0.04 \mathrm{GeV}^{2} / c^{2}$ und die mit ihnen verknüpften systematischen Fehler zu erkennen.

Weitere Fortschritte wurden bei der Ereignisauswahl erzielt. Während die erste Analyse auch Ereignisse aus der Runperiode von 8000 bis 9999 umfaßte, in welchen das Verhältnis von elastischen zu unelastischen Ereignissen ein Vielfaches kleiner ist und durch welche das Spektrum im Bereich hoher $\mathrm{Q}^{2}$ angehoben wird, so ist es in der erneuten Analyse gelungen ohne die Ereignisse aus diesen frühen Runs ein vergleichbares Ergebnis zu erzielen.

Der Unterschied im statistischen Fehler beruht auf dem kleineren $\mathrm{Q}^{2}$-Intervall, insbesondere der unteren Fitgrenze, auf dem der Fit ausgeführt werden kann. Die hiermit verbundenen systematischen Probleme wurden in der ersten Analyse nicht erkannt. 


\subsection{Ergebnis der Analyse}

Das Ergebnis der Analyse der 215 Millionen aufgezeichneten Daten der im September 1997 beendeten Meßzeit liefert einen mittleren quadratischen Ladungsradius von

$$
\left\langle r^{2}\right\rangle=0.42 \pm 0.06 \text { (stat) } \pm 0.08 \text { (syst) } \mathrm{fm}^{2},
$$

der in einem $\mathrm{Q}^{2}$ Bereich von 0.04 bis $0.1725 \mathrm{GeV}^{2} / c^{2}$ und auf 24118 elastischen PionElektron-Streuereignissen beruhend gemessen wurde. Diese Wert ist in guter Übereinstimmung mit dem von Amendolia et al. [4] in dem $\mathrm{Q}^{2}$-Intervall von $0.014<\mathrm{Q}^{2}<$ $0.122 \mathrm{GeV}^{2} / c^{2}$ gemessenen Radius von

$$
\left\langle r^{2}\right\rangle=0.432 \pm 0.016 \mathrm{fm}^{2}
$$

Abbildung 6.1 zeigt einen Vergleich der gemessenen Formfaktoren von Amendolia et al. und dieser Arbeit.

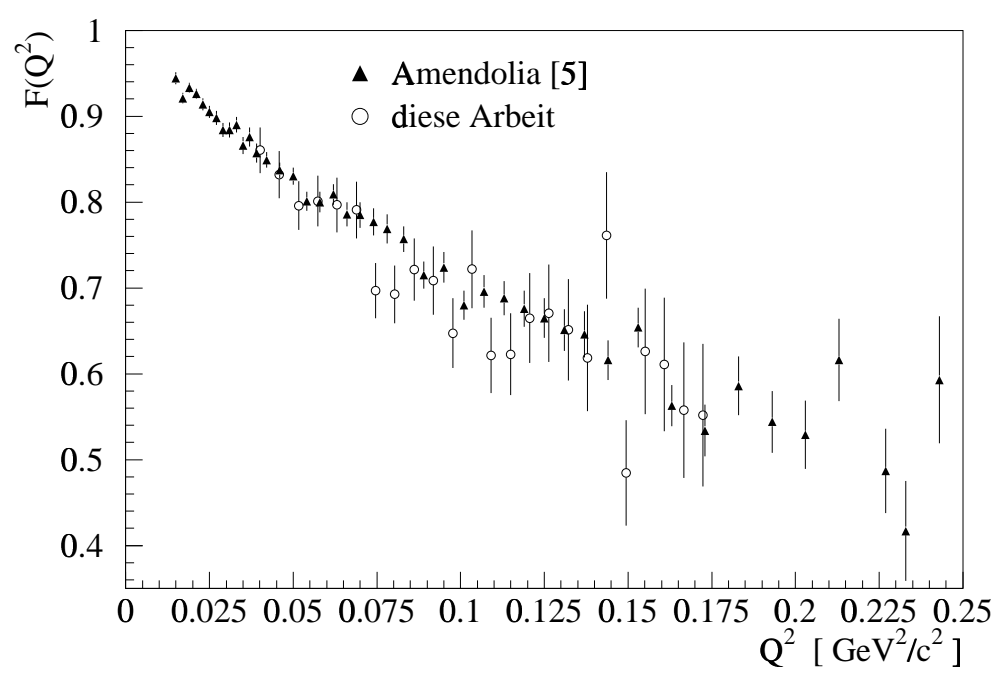

Abbildung 6.1: Vergleich des Formfaktors mit Literaturwert von Amendolia et al.[4]. Die Fehler sind jeweils ohne Berücksichtigung der systematischen Fehler. Eingezeichnet sind nur jene Datenpunke, welche zur Messung des elektromagnetischen Ladungsradius benutzt wurden. 


\section{A Glossar}

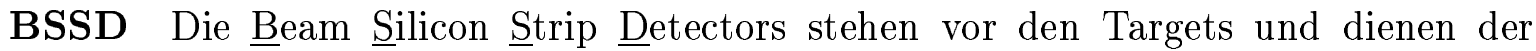
Spurvermessung einlaufender Strahlteilchen (s. Seite 19).

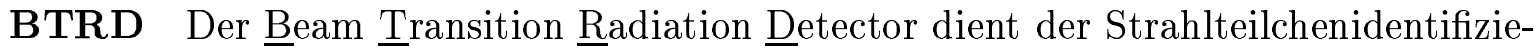
rung und ist vor dem Vertex-Bereich angebracht (s. Seite 19 und 25).

DSD Doublesided Strip Detector (s. Seite 22).

ETRD Der Electron Transition Radiation Detector dient besonders der Identifizierung von Elektronen und Pionen. Der ETRD steht im M2-Spektrometer vor dem RICH (s. Seite 25).

HSD Drei Silizium-Mikrostreifen-Detektoren, je einer vor und hinter dem BTRD und der dritte hinter dem Vertex-Spektrometer, sind mit dem HST verschaltet und werden

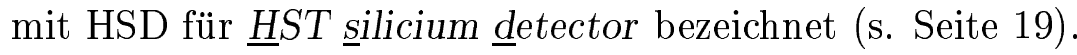

HST Der $\underline{h}$ ardware s scatter trigger wird im Primakofffilter zur event-Auswahl benutzt. Er besteht aus den drei HSDs (s. Seite 19).

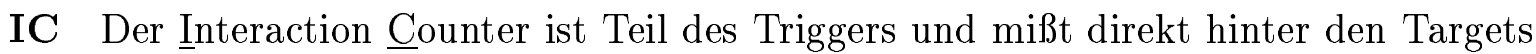
die Multiplizität des Ereignisses (s. Seite 28).

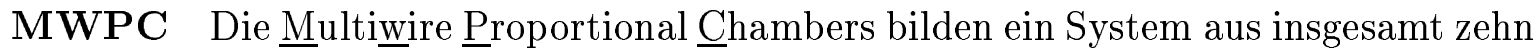
Stationen, die über die Spektrometer M2 (sieben Stationen) und M3 (drei Stationen) verteilt sind.

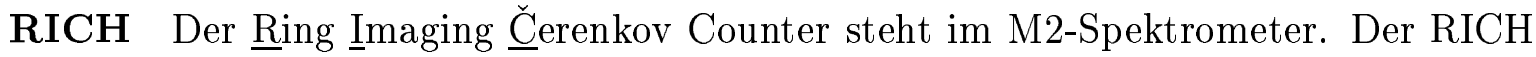
trennt vor allem zwischen Protonen, Kaonen und Pionen (s. Seite 26).

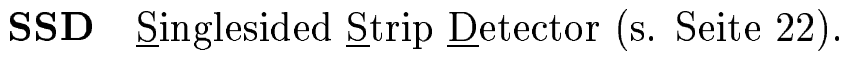

Tevatron Das Tevatron ist das $6.28 \mathrm{~km}$ lange Synchrotron des Fermilabs. Es liefert Protonen mit einem Impuls von rund $800 \mathrm{GeV} / c$.


und M3-Spektrometer und ermöglichen den Nachweis langlebiger Hyperonen durch ihre Zerfälle (s. Seite 23). 


\section{B Feynman Diagramme der elastischen Streuung}
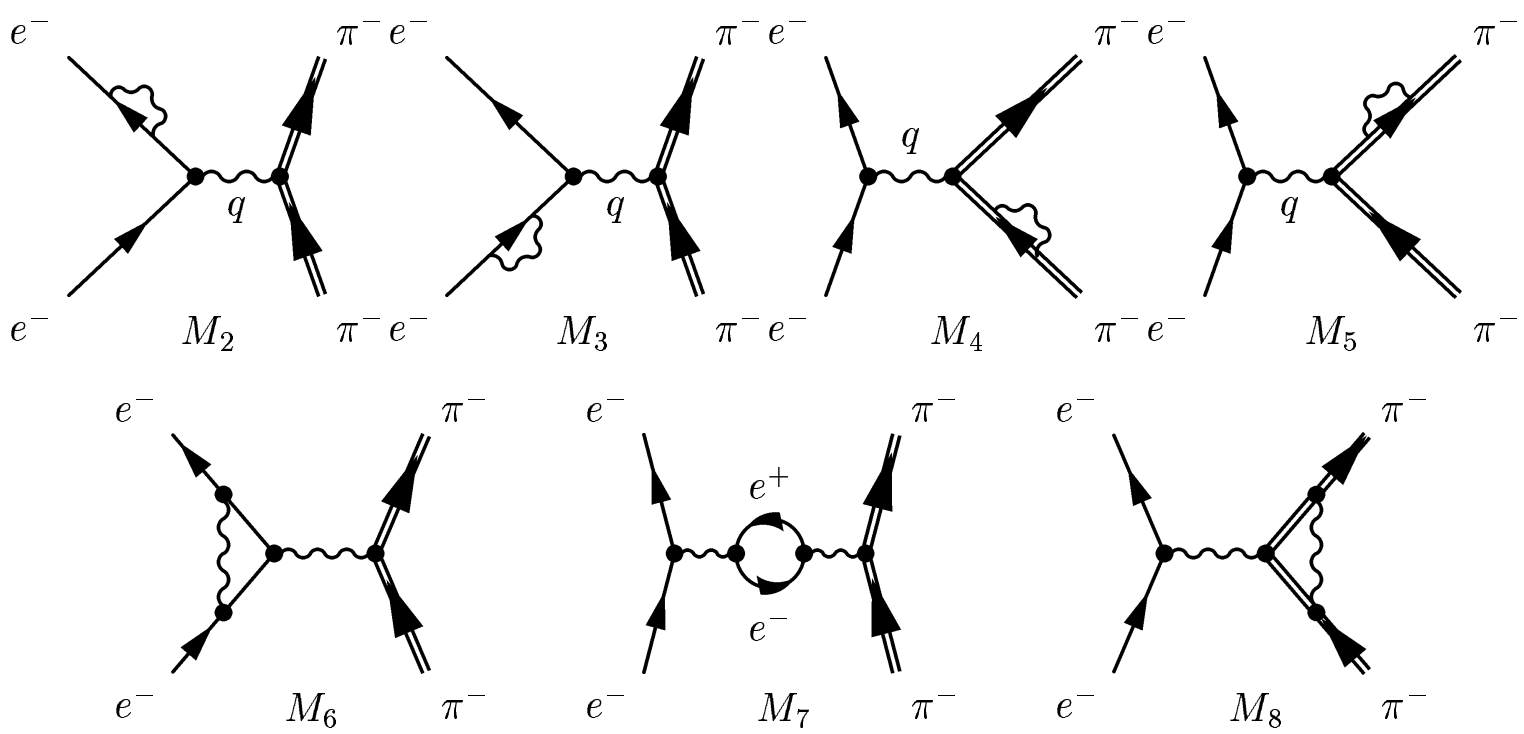

Abbildung B.2: Feynmangraphen zur Selbstenergie $\left(M_{2}-M_{5}\right)$ Vakuumpolarisation $\left(M_{7}\right)$ und zur Vertexkorrektur $\left(M_{6}\right.$ und $\left.M_{8}\right)$.
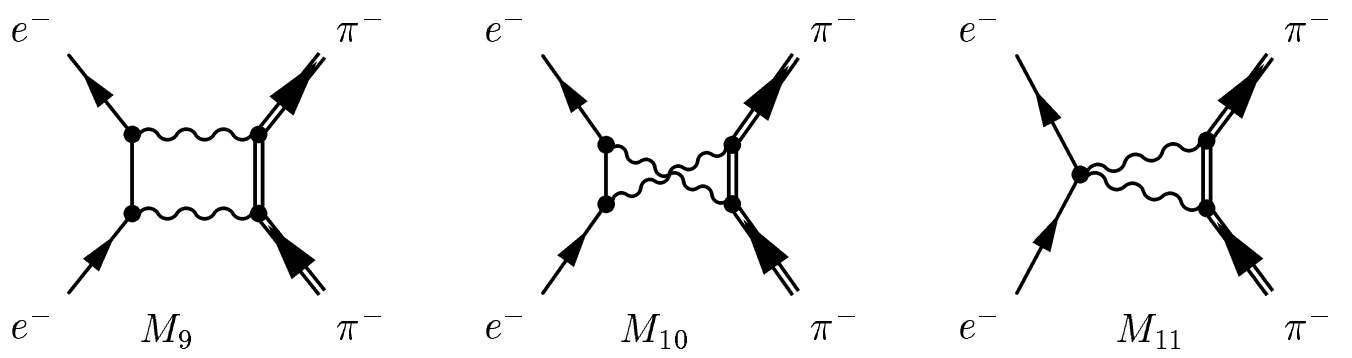

Abbildung B.3: Feynmangraphen zum Zwei-Photonen-Austausch.

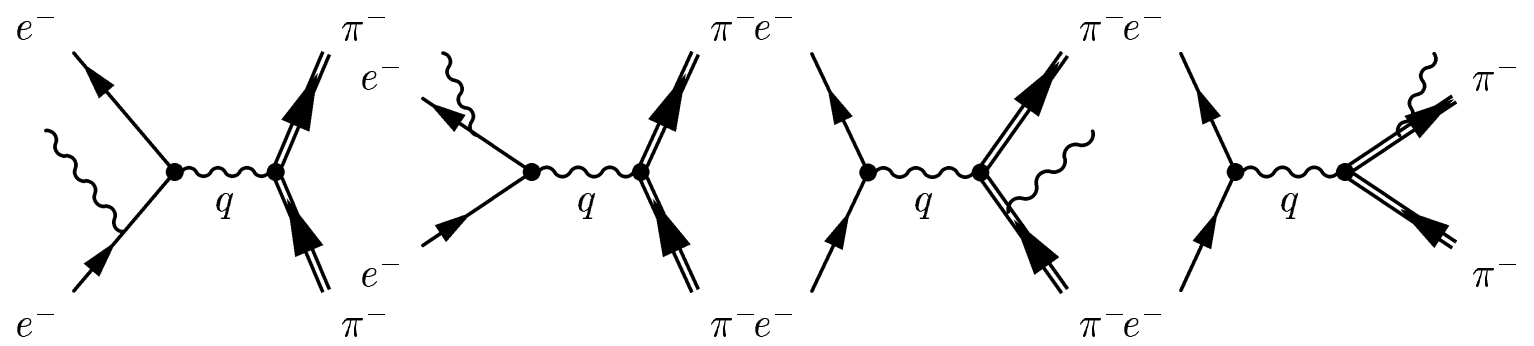

Abbildung B.4: Feynmangraphen zur internen Bremsstrahlung von Elektronen und Pionen. 


\section{Notationen}

Tabelle C.1: Übersicht verwendeter Symbole in den Formeln zur Wechselwirkung von Strahlung mit Materie.

\begin{tabular}{cll}
\hline Symbol & Definition & Einheit, bzw. Wert \\
\hline$\alpha$ & Feinstrukturkonstante & $17137,0359895(61)$ \\
$M$ & Masse des einlaufenden Teilchens & $\mathrm{MeV} / c^{2}$ \\
$E$ & Energie des einlaufenden Teilchens $\gamma M c^{2}$ & $\mathrm{MeV}$ \\
$T$ & Kinetische Energie & $\mathrm{MeV}$ \\
$m_{e} c^{2}$ & Elektronenmasse $\times c^{2}$ & $0.51099906(15) \mathrm{MeV}$ \\
$r_{e}$ & klassischer Elektronenradius & $2.81794092(38) \mathrm{fm}$ \\
& $e^{2} / 4 \pi \varepsilon_{0} m_{e} c^{2}$ & \\
$N_{A}$ & Avogadro Zahl & $6.0221367(36) \times 10^{23} \mathrm{~mol}^{-1}$ \\
$z e$ & Ladung des einlaufenden Teilchens & \\
$Z$ & Kernladungszahl des Materials & \\
$A$ & Massen des Materials & $0.307075 \mathrm{MeV} \mathrm{g}^{-1} \mathrm{~cm}^{2}$ \\
$K / A$ & $4 \pi N_{A} r_{e}^{2} m_{e} c^{2} / A$ & für $A=1 \mathrm{~g} \mathrm{~mol}^{-1}$ \\
& & $\mathrm{eV}$ \\
$I$ & Mittlere Ionisationsenergie & $\mathrm{g} \mathrm{cm}-2$ \\
$\delta$ & Korrekturterm des Dichteeffekts & $\mathrm{g} / \mathrm{cm}^{3}$ \\
$X_{0}$ & Strahlungslänge & $\mathrm{cm}$ \\
$\rho$ & Dichte des betreffenden Materials &
\end{tabular}




\section{Hodoskop 1 Daten}

Tabelle D.2: $x$-Positionen und Effizienzen der Hodoskop 1 Elemente.

\begin{tabular}{|c|c|c|c|c|c|}
\hline Name & Breite & $x$-Mitte & $\begin{array}{l}\text { Effizienz } \\
\text { gemessen }\end{array}$ & $x$-Position Lücke & $\begin{array}{l}\text { Effizienz } \\
\text { angepaßt }\end{array}$ \\
\hline \multicolumn{6}{|c|}{ H1 positive Elemente (H1+ Diskriminator) } \\
\hline m2_th1_01 & $25 \mathrm{~mm}$ & $27.42 \mathrm{~cm}$ & $100.0-2.0+0.0$ & $26.17-26.14 \mathrm{~cm}$ & \\
\hline m2_th1_02 & $25 \mathrm{~mm}$ & $24.89 \mathrm{~cm}$ & $94.9-2.4+1.5$ & $23.64-23.62 \mathrm{~cm}$ & \\
\hline m2_th1_03 & $25 \mathrm{~mm}$ & $22.37 \mathrm{~cm}$ & $93.9-1.8+1.5$ & $21.12-21.07 \mathrm{~cm}$ & \\
\hline m2_th1_04 & $25 \mathrm{~mm}$ & $19.82 \mathrm{~cm}$ & $96.0-1.2+1.0$ & $18.57-18.56 \mathrm{~cm}$ & \\
\hline m2_th1_05 & $20 \mathrm{~mm}$ & $17.56 \mathrm{~cm}$ & $93.3-1.5+1.3$ & $16.56-16.54 \mathrm{~cm}$ & \\
\hline m2_th1_06 & $20 \mathrm{~mm}$ & $15.54 \mathrm{~cm}$ & $93.9-1.2+1.1$ & $14.54-13.52 \mathrm{~cm}$ & \\
\hline m2_th1_07 & $20 \mathrm{~mm}$ & $13.52 \mathrm{~cm}$ & $93.8-1.0+1.0$ & $12.52-12.43 \mathrm{~cm}$ & \\
\hline m2_th1_08 & $20 \mathrm{~mm}$ & $11.43 \mathrm{~cm}$ & $94.7-0.9+0.8$ & $10.43-10.41 \mathrm{~cm}$ & \\
\hline m2_th1_09 & $15 \mathrm{~mm}$ & $9.66 \mathrm{~cm}$ & $90.0-1.2+1.1$ & $8.91-8.91 \mathrm{~cm}$ & \\
\hline m2_th1_10 & $15 \mathrm{~mm}$ & $8.16 \mathrm{~cm}$ & $84.7-1.4+1.2$ & $7.41-7.39 \mathrm{~cm}$ & \\
\hline m2_th1_11 & $15 \mathrm{~mm}$ & $6.64 \mathrm{~cm}$ & $94.9-0.7+0.6$ & $5.88 \mathrm{~cm}$ & \\
\hline m2_th1_12 & $15 \mathrm{~mm}$ & $5.13 \mathrm{~cm}$ & $96.0-0.6+0.6$ & $4.38-4.32 \mathrm{~cm}$ & \\
\hline m2_th1_13 & $15 \mathrm{~mm}$ & $3.57 \mathrm{~cm}$ & $96.3-0.9+0.7$ & $2.83-2.77 \mathrm{~cm}$ & \\
\hline m2_th1_14 & $10 \mathrm{~mm}$ & $2.27 \mathrm{~cm}$ & $90.8-1.3+1.2$ & $1.77-\quad 1.69 \mathrm{~cm}$ & \\
\hline \multicolumn{6}{|c|}{ H1 neutrale Elemente (H10 Diskriminator) } \\
\hline m2_th1_15 & $10 \mathrm{~mm}$ & $1.19 \mathrm{~cm}$ & $97.8-0.3+0.2$ & $0.65 \mathrm{~cm}$ & 59.0 \\
\hline m2_th1_16 & $10 \mathrm{~mm}$ & $0.15 \mathrm{~cm}$ & $96.7-1.9+1.9$ & $-0.35--0.38 \mathrm{~cm}$ & 45.0 \\
\hline m2_th1_17 & $5 \mathrm{~mm}$ & $-0.63 \mathrm{~cm}$ & $83.3-1.8+1.7$ & $-0.88--0.94 \mathrm{~cm}$ & 40.0 \\
\hline m2_th1_18 & $5 \mathrm{~mm}$ & - $1.19 \mathrm{~cm}$ & $85.6-1.6+1.5$ & $-1.44--1.46 \mathrm{~cm}$ & 56.0 \\
\hline m2_th1_19 & $5 \mathrm{~mm}$ & $-1.71 \mathrm{~cm}$ & $90.7-2.8+2.8$ & $-1.96--2.06 \mathrm{~cm}$ & 71.0 \\
\hline m2_th1_20 & $5 \mathrm{~mm}$ & $-2.31 \mathrm{~cm}$ & $68.4-2.7+2.6$ & $-2.56--2.64 \mathrm{~cm}$ & 72.0 \\
\hline m2_th1_21 & $5 \mathrm{~mm}$ & $-2.89 \mathrm{~cm}$ & $92.9-1.3+1.1$ & $-3.14--3.17 \mathrm{~cm}$ & 113.0 \\
\hline m2_th1_22 & $5 \mathrm{~mm}$ & $-3.42 \mathrm{~cm}$ & $93.4-1.5+1.3$ & $-3.67--3.71 \mathrm{~cm}$ & 88.0 \\
\hline \multicolumn{6}{|c|}{ H1 negative Elemente (H1- Diskriminator) } \\
\hline m2_th1_23 & $10 \mathrm{~mm}$ & $-4.21 \mathrm{~cm}$ & $92.5-1.3+1.1$ & $-4.71--4.75 \mathrm{~cm}$ & 71.0 \\
\hline m2_th1_24 & $10 \mathrm{~mm}$ & $-5.25 \mathrm{~cm}$ & $98.2-0.2+0.2$ & $-5.75--5.77 \mathrm{~cm}$ & 67.0 \\
\hline m2_th1_25 & $10 \mathrm{~mm}$ & $-6.27 \mathrm{~cm}$ & $94.2-0.5+0.5$ & $-6.77--6.78 \mathrm{~cm}$ & 63.0 \\
\hline m2_th1_26 & $15 \mathrm{~mm}$ & $-7.53 \mathrm{~cm}$ & $95.9-0.5+0.4$ & $-8.28--8.30 \mathrm{~cm}$ & 73.0 \\
\hline m2_th1_27 & $15 \mathrm{~mm}$ & $-9.05 \mathrm{~cm}$ & $95.5-0.7+0.6$ & $-9.80--9.90 \mathrm{~cm}$ & 71.0 \\
\hline m2_th1_28 & $15 \mathrm{~mm}$ & $-10.65 \mathrm{~cm}$ & $93.7-0.9+0.8$ & $-11.40--11.40 \mathrm{~cm}$ & 73.0 \\
\hline m2_th1_29 & $15 \mathrm{~mm}$ & $-12.15 \mathrm{~cm}$ & $91.5-1.2+1.1$ & $-12.90--12.94 \mathrm{~cm}$ & 75.0 \\
\hline m2_th1_30 & $15 \mathrm{~mm}$ & - $13.69 \mathrm{~cm}$ & $92.6-1.2+1.1$ & $-14.44--14.47 \mathrm{~cm}$ & 80.0 \\
\hline m2_th1_31 & $20 \mathrm{~mm}$ & $-15.47 \mathrm{~cm}$ & $93.9-1.1+0.9$ & $-16.47--16.50 \mathrm{~cm}$ & 81.0 \\
\hline m2_th1_32 & $20 \mathrm{~mm}$ & $-17.50 \mathrm{~cm}$ & $94.3-1.2+1.0$ & $-18.50--18.58 \mathrm{~cm}$ & 55.0 \\
\hline m2_th1_33 & $20 \mathrm{~mm}$ & $-19.58 \mathrm{~cm}$ & $80.2-2.5+2.3$ & $-20.58--20.65 \mathrm{~cm}$ & 90.0 \\
\hline m2_th1_34 & $20 \mathrm{~mm}$ & $-21.65 \mathrm{~cm}$ & $86.9-2.3+2.0$ & $-22.65--22.73 \mathrm{~cm}$ & 95.0 \\
\hline m2_th1_35 & $25 \mathrm{~mm}$ & $-23.98 \mathrm{~cm}$ & $95.3-1.5+1.1$ & $-25.23--25.28 \mathrm{~cm}$ & 103.0 \\
\hline m2_th1_36 & $25 \mathrm{~mm}$ & $-26.53 \mathrm{~cm}$ & $94.4-1.9+1.5$ & $-27.78--27.82 \mathrm{~cm}$ & 85.0 \\
\hline m2_th1_37 & $25 \mathrm{~mm}$ & $-29.07 \mathrm{~cm}$ & $92.0-3.2+2.1$ & $-30.32--30.37 \mathrm{~cm}$ & 60.0 \\
\hline m2_th1_38 & $25 \mathrm{~mm}$ & $-31.62 \mathrm{~cm}$ & $100.0-3.1+0.0$ & $-32.87-$ & \\
\hline
\end{tabular}




\section{Abbildungsverzeichnis}

2.1 Kinematik der Pion-Elektron-Streuung im Laborsystem . . . . . . . . . 3

2.2 Kinematik der Pion-Elektron-Streuung im Schwerpunktsystem . . . . . 4

$2.3 \mathrm{Q}^{2}$-Abhängigkeit verschiedener Observablen der Pion-Elektron-Streuung 6

2.4 Feynmandiagramm der elastischen Sigma-Elektron-Streuung . . . . . . 7

2.5 Mott-Wirkungsquerschnitt und der Formfaktor für verschiedene Radien 9

2.6 Feynmandiagramm 1. Ordnung der Born'sche Näherung der Pion-Elektron-

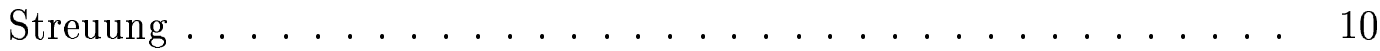

2.7 Beiträge der einzelnen Strahlungskorrekturen . . . . . . . . . . . . 11

2.8 Strahlungskorrekturen für einen Cut-Off-Parameter von $100 \mathrm{GeV} / c$. . 12

2.9 Abhängigkeit der Gesamt-Strahlungskorrektur vom Cut-Off-Parameter 13

3.1 Die SU(4)-Multipletts der Baryonen aus den vier leichtesten Quarks . . 15

3.2 SELEX Layout . . . . . . . . . . . . . . . . . . 18

3.3 Layout des Hyperonen-Kanal . . . . . . . . . . . . . . . . 19

3.4 Layout der Beam-Vertex Region . . . . . . . . . . . . . . 20

3.5 Strahlimpulsverteilung und eine Zahl der einlaufenden Strahlteilchen pro

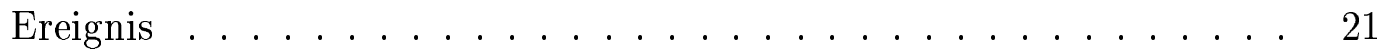

3.6 Layout des M1-Spektrometers . . . . . . . . . . . . 22

3.7 Layout des M2-Spektrometers . . . . . . . . . . . . . 23

3.8 Layout des M3-Spektrometers . . . . . . . . . . . . . 24

3.9 Strahlteilchenidentifikation über die Zahl der aktiven BTRD Ebenen . . 25

3.10 Verteilung der ETRD Cluster für Elektronen und Pionen . . . . . . . . 26

3.11 Impulsabhängigkeit des Čerenkov winkels für verschiedene Teilchen . 27

3.12 Energiedeposition im Wechselwirkungszähler . . . . . . . . . . . . . 29

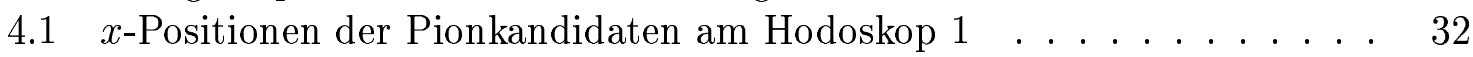

$4.2 x$-Positionsvermessung des Hodoskop 1 am Beispiel des Elements 16 . . 34

$4.3 y$-gegen $x$-Position der Spuren am Element 17 des Hodoskops $1 \ldots 34$

4.4 Effizienzmessung der Hodoskop 1 Elemente . . . . . . . . . . . . . 35

4.5 Impulsübertrag gegen die Elektron und Pion $x$-Position am Hodoskop 1 für simulierte Ereignisse (GEANT) . . . . . . . . . . . . 36

4.6 Die $x$-Positionsspektrum der Elektronen und Pionen an Hodoskop $1 \mathrm{im}$ Vergleich zwischen GEANT und Daten jeweils für die gemessenen Hodoskopeffizienzen und für die an die Verteilung der Daten angepaßten Effizienzen . . . . . . . . . . . . . . . .

4.7 Die Triggerakzeptanz für angepaßte und gemessene Hodoskopeffizienzen, wie sie sich aus simulierten Ereignissen im $\mathrm{Q}^{2}$-Intervall von 0.02 bis $0.3 \mathrm{GeV}^{2} / c^{2}$ ergibt . . . . . . . . . . . . . . . 39

4.8 GEANT erzeugtes $\mathrm{Q}^{2}$-Spektrum . . . . . . . . . . . . . 41

4.9 Soap rekonstruiertes $Q^{2}$-Spektrum der GEANT Simulation . . . . . . . 42

4.10 Anteile der verschiedenen Verluste an den Akzeptanzverlusten . . . . . 42

4.11 Mit der Triggerwahrscheinlichkeit (gemessene Effizienzen) gewichtetes von Soap rekonstruiertes $Q^{2}$-Spektrum der GEANT Simulation . . . . . 43

5.1 Impuls gegen normalisierte Likelihoodfunktion der Elektronen . . . . . 47

5.2 Vertex z-Positionen .............................. 49

5.3 Winkel-Winkel-Korrelation für verschiedene Schnitte . . . . . . . . 51 
5.4 Pull des Elektron- und des Pionwinkels sowie die Vertex-Elastizität für die Daten aus dem Strip 2 und die im Radiusfit verwendeten Ereignisse 52

5.5 Auflösungen der verwendeten Observablen . . . . . . . . . . . . 53

5.6 Abhängigkeiten der Auflösung des Elektronstreuwinkels . . . . . . . . 54

5.7 Vom gemessen $\mathrm{Q}^{2}$-Spektrum zum Radiusfit . . . . . . . . . . . . 55

5.8 Abhängigkeit des Radius von den Schnitten . . . . . . . . . . . 56

5.9 Abhängigkeit der Radius von den Fitgrenzen . . . . . . . . . . . . 57

6.1 Vergleich des Formfaktors mit dem Literaturwert von Amendolia et al. [4] 61

B.2 Feynmangraphen zur Selbstenergiem Vakuumpolarisation und Vertex-

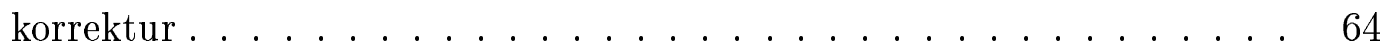

B.3 Feynmangraphen zum Zwei-Photonen-Austausch . . . . . . . . . . . 64

B.4 Feynmangraphen zur interen Bremsstrahlung . . . . . . . . . . 64 


\section{Tabellenverzeichniss}

1.1 Eigenschaften der Quarks . . . . . . . . . . . . . 2

$2.1 \mathrm{Q}_{\text {max }}^{2}$ für verschiedene Strahlteilchen im SELEX Experiment . . . . . 5

3.1 Eigenschaften von charm-Baryonen . . . . . . . . . . . 16

3.2 Spezifikationen der elektromagnetischen Kalorimeter . . . . . . . . . . 28

3.3 Spezifikationen der Hodoskope . . . . . . . . . . . . . . 30

3.4 Die Zeitpunkte der verschiedenen Trigger-Updates . . . . . . . . . . . . 31

5.1 Kenngrößen der Targets . . . . . . . . . . . . . . . 45

5.2 Statistik der Datenreduktionsstufen Strip 1 und $2 \ldots \ldots$. . . . . 46

5.3 Statistik der Datenanalyse . . . . . . . . . . . . . . 52

5.4 Tabelle der systematischen Fehler . . . . . . . . . . . . . . 59

C.1 Übersicht verwendeter Symbole in den Formeln zur Wechselwirkung von Strahlung mit Materie . . . . . . . . . . . . . . . 6 65

D.2 $x$-Positionen und Effizienzen der Hodoskop 1 Elemente. . . . . . . 66 


\section{Literaturverzeichnis}

[1] Particle Data Group, http://pdg.lbl.gov/ (1999).

[2] R. Hofstadter, Rev Mod Phys 28 (1956).

[3] S. Amendolia et al., Phys Lett B 178 (1986).

[4] S. Amendolia et al., Phys Lett B 146, 116 (1984).

[5] Povh, Rith, Scholz, and Zetsche, Teilchen und Kerne, Springer Verlag, 1996.

[6] J. Bjorken and S. Drell, Relativistische Quantenmechanik, BI-Wissenschaftsverlag, 1990.

[7] W. Greiner and J. Reinhartd, Theoretische Physik, Verlag Harri Deutsch, 1987.

[8] M. Rosenbluth, Phys Rev 79, 615 (1950).

[9] R. Sachs, Phys Rev 126, 2256 (1962).

[10] J. Kahane, Phys Rev 135, 975 (1964).

[11] L. Maximon, User's Manual for Radiative Corrections, Department of Physics, George Washington University, 1993.

[12] D. Bardin, V. Semikoz, and N. Shumeiko, Sov J Nucl Phys 10, 586 (1970).

[13] J. Russ et al., A proposal to construct Selex, Proposal P781, Fermilab, 1987.

[14] Particle Data Group, Phys Rev D (1998).

[15] P. Cooper, J. Grunhaus, and J. Russ, Search for doubly charmed baryons using $600 \mathrm{GeV} / \mathrm{c}$ hyperon and pion beams, H-Note 718, SELEX Internal Report, 1994.

[16] J. Russ, Charm baryon physics - present and future, H-Note 695, SELEx Internal Report, 1994, Invited talk at Charm2000 Meeting at Fermilab.

[17] H. Siebert, Nucl Phys B 21, 183 (1991).

[18] M. Moinester, D. Ashery, L. Landsberg, and H. Lipkin, Pentaquark search with energetic hadron beams, H-Note 708, SELEx Internal Report, 1994, presented at CHARM2000 Workshop, Fermilab / hep-ph/9407319.

[19] H. Siebert, Nucl Phys B 21, 270 (1991).

[20] S. Paul, Nucl Phys B 21 (1991).

[21] H. Primakoff, Phys Rev 81, 899 (1951).

[22] U. Dersch, Messung totaler Wirkungsquerschnitte mit Sigma-, Proton, Pi- und Pi+ bei $600 \mathrm{GeV} / \mathrm{c}$ Laborimpuls, PhD thesis, Universität Heidelberg, 1999. 
[23] B. Povh, Nucl Phys A 532, 133c (1991).

[24] K. Königsmann, S. Paul, and B. Povh, Electromagnetic radii of hyperons using Selex, H-Note 574, Selex Internal Report, 1994.

[25] P. Pogodin et al., Drift chambers of the M1 spectrometer: the detector, electronics, and software, H-Note 788, SELEX Internal Report, 1997.

[26] W. Leo, Techniques for Nuclear and Particle Physics Experiments, Springer Verlag, second edition, 1994.

[27] K. Königsmann, S. Masciocchi, and K. Vorwalter, The Large Angle Silicon Detectors (LASD) for E781, H-Note 661, SELEX Internal Report, 1993.

[28] T. Lungov and L. Emediato, Vector drift chambers database, H-Note 779, Selex Internal Report, 1997.

[29] N. Bondar et al., E781 beam transition radiation detector, H-Note 746, SELEX Internal Report, 1995.

[30] N. Terentyev, TRD Efficiency and Rejection in E781 Monte Carlo, H-Note 745, SELEX Internal Report, 1995.

[31] A. Antonov, G. Dzyubenko, and M. Kubantsev, Geometry of the assembled E781 PHOTON 1,2 detectors, H-Note 748, Selex Internal Report, 1995.

[32] J. Engelfried et al., The E781 trigger and data aquisition system, H-Note 643, SELEX Internal Report, 1995.

[33] A. Kulyavtsev et al., E781 hardware trigger preliminary design, 1994.

[34] L. Landau, J. Phys. (USSR) 8, 201 (1944).

[35] H. Krüger, Multiplizitätstrennung mit dem Wechselwirkungszähler im SELEXExperiment, Diplomarbeit, MPI f. Kernphysik / Univ. Heidelberg, 1996.

[36] I. Eschrich, H. Krüger, J. Simon, and K. Vorwalter, The hadron-electron elastic scattering trigger, H-Note 803, SELEX Internal Report, 1997.

[37] N. Kouropatkine, Generalized likelihood method for lepton identification in selex, H-Note 806, Selex Internal Report, 1998.

[38] K. Vorwalter, Determination of the Pion Charge Radius with a Silicon Microstrip Detector System, Dissertation, MPI f. Kernphysik / Univ. Heidelberg, 1998.

[39] I. Eschrich, Measurement of the $\Sigma^{-}$Charge Radius at the Fermilab Hyperon Beam, Dissertation, MPI f. Kernphysik / Univ. Heidelberg, 1998. 


\section{Danksagung}

An dieser Stelle möchte ich bei all denen bedanken, die zur Entstehung dieser Arbeit und der hiermit verbundenen Erfahrungen beigetragen haben.

Prof. Bogdan Povh danke ich für die Gelegenheit zur Durchführung dieser Arbeit am Max-Planck-Institut für Kernphysik und für die hiermit verbundenen Erfahrungen, eine große internationale Forschungseinrichtung kennen zu lernen.

Henning Krüger und Jürgen Simon danke ich, da sie zum Gelingen dieser Arbeit nicht nur in wissenschaftlicher Hinsicht viel beigetragen haben, die zu immer wieder geduldig bei der Lösung meiner Fragen halfen und mit denen nicht nur die Zusammenarbeit immerwieder viel Spaß bereitete.

Ivo Eschrich und Klaus Vorwalter habe ich besonders für deren Hilfestellungen während der Anfangsphase und für deren weitreichenden Vorarbeiten, auf denen diese Arbeit fußt, zu danken.

Weiterhin bin ich zu Dank verpflichtet: Nikolai Kuropatkin, während dessen Zeit am MPI die Arbeiten am GEANT große Fortschritte machten, Yuri Scheglov, der viele Probleme der Strahlungskorrekturen löste, sowie all jenen die gerne bei kleineren oder auch größeren Problemen halfen, als da wären Christian Busch, Uwe Dersch, Douglas Jansen, Thomas Nunnemann, Prov.-Doz. Dr. Josef Pochodzalla, Tina Schati, Claudia Schröder 

Erklärung:

Ich versichere, dass ich diese Arbeit selbständig verfaßt und keine anderen als die angegebenen Quellen und Hilfsmittel benutzt habe.

Heidelberg, den 14. Oktober 1999 